\title{
Statistical Inference on Panel Data Models: A Kernel Ridge Regression Method
}

\author{
Shunan Zhao ${ }^{1}$ Ruiqi Liu ${ }^{2}$ Zuofeng Shang*2 \\ ${ }^{1}$ Department of Economics, Binghamton University \\ ${ }^{2}$ Department of Mathematical Sciences, Binghamton University
}

\begin{abstract}
We propose statistical inferential procedures for panel data models with interactive fixed effects in a kernel ridge regression framework. Compared with traditional sieve methods, our method is automatic in the sense that it does not require the choice of basis functions and truncation parameters. Model complexity is controlled by a continuous regularization parameter which can be automatically selected by generalized cross validation. Based on empirical processes theory and functional analysis tools, we derive joint asymptotic distributions for the estimators in the heterogeneous setting. These joint asymptotic results are then used to construct confidence intervals for the regression means and prediction intervals for the future observations, both being the first provably valid intervals in literature. Marginal asymptotic normality of the functional estimators in homogeneous setting is also obtained. Simulation and real data analysis demonstrate the advantages of our method.
\end{abstract}

Keywords and Phrases: kernel ridge regression, panel data models with interactive fixed effects, joint asymptotic distribution, empirical processes, Functional Bahadur representation JEL Classification: C13, C14, C23

\section{Introduction}

Panel data models with interactive fixed effects (IFE) have many applications in econometrics and statistics, e.g., individuals' education decision (Carneiro et al., 2003; Cunha et al., 2005), house price analysis (Holly et al., 2010), prediction of investment returns (Eberhardt et al., 2013), risk-return relation (Ludvigson and Ng, 2009, 2016), etc. The IFE can capture both cross section dependence and heterogeneity in the data, which makes these models more flexible than the classic fixed or random effect models. Cross section dependence is usually characterized by time-varying common factors, and heterogeneity is captured by individual-specific factor loadings. There has been an increasing literature in this area addressing various statistical inferential problems. Earlier studies focused on parametric settings; see Pesaran (2006), Bai and Ng (2006), Bai (2009), Moon and Weidner (2010), Moon and Weidner (2015), among others. A common crucial assumption in these papers is that the response and predictor variables are linearly related and the parameters of interest are finite-dimensional.

\footnotetext{
*Corresponding author. Assistant Professor at Department of Mathematical Sciences, Binghamton University. Address: PO Box 6000, Binghamton, New York 13902-6000, USA; Email: zshang@binghamton.edu; Tel: (607)7774263; Fax: (607)777-2450. Research Sponsored by a start-up grant from Binghamton University.
} 
Recently, efforts were devoted to nonparametric/semiparametric panel data models with IFE. For instance, Freyberger (2012), Su and Jin (2012), Jin and Su (2013) and Su and Zhang (2013), $\mathrm{Su}$ et al. (2015), among others, proposed sieve methods for estimating or testing the infinitedimensional regression functions; Huang (2013) and Cai et al. (2016) proposed local polynomial methods, differing from sieves by their local feature. The success of the sieve methods hinges on a good choice of basis functions; see Chen (2007) for a comprehensive introduction. The truncation parameter, i.e., number of basis functions used for model fitting, changes in a discrete fashion, which may yield an imprecise control on the model complexity, as pointed out in Ramsay and Silverman (2005). For these reasons, it is worthwhile to explore a method that relies less on the choice of basis or the choice of discrete truncation parameters, which will possess computational, theoretical and conceptual advantages.

In this paper, we propose a new kernel-based nonparametric method, called as kernel ridge regression (KRR), for handling panel data models. Our method relies on the assumption that the regression function belongs to a reproducing kernel Hilbert space (RKHS) driven by a kernel function called as reproducing kernel. The KRR estimator is "basis-free" in the sense that it can be explicitly expressed by the kernels rather than the basis functions. Our method is applicable to a broad class of RKHS, e.g., Euclidean space, Sobolev space, Gaussian kernel space, or spaces with advanced structures such as semiparametric/additive structures; see Wahba (1990), Shang and Cheng (2013), Zhao et al. (2016) about more descriptions of these spaces. Reproducing kernels corresponding to the above mentioned RKHS have (approximately) explicit expressions which can be directly used in our inferential procedures. For applications of RKHS in other fields such as statistical machine learning, see Hofmann et al. (2008).

In contrast to the method of sieves, our KRR method does not involve the discrete truncation parameter. Instead, it directly searches the estimator in the entire (possibly infinite-dimensional) function spaces, though the process of searching requires the use of a continuous regularization parameter which controls the smoothness of the estimators. In smoothing splines or KRR, regularization parameters are usually selected by generalized cross validation (GCV); see Craven and Wahba (1978), Wahba (1990), Gu (2013), Wang (2011). The selection procedure proceeds by searching a global minimizer of the GCV criteria function which provides a more accurate management on model complexity. As a result, the estimator may yield better performance as observed by Shang and Cheng (2015) in functional data analysis. In this paper, we adapt the traditional GCV criteria to semiparametric panel data models in both heterogeneous and homogeneous settings. The selection algorithms are easy-to-use with satisfactory performance as demonstrated in our simulation study and real data analysis (Section 5).

Besides numerical advantages, the proposed method is theoretically powerful. For instance, based on our RKHS framework, it is theoretically more convenient to derive the joint asymptotic distributions for the estimators of the linear and nonlinear components; see Theorems 3.3 and 3.4. Nonetheless, joint asymptotic distributions are more difficult to prove in the sieve or local 
polynomial framework. Our joint asymptotic results can be used to design novel statistical procedures such as confidence intervals for the regression means and prediction intervals for the future observations, though they can naturally imply the marginal asymptotic results obtained by $\mathrm{Su}$ and Jin (2012) as a corollary. The theory developed in this paper relies on nonstandard technical tools such as empirical processes theory and functional analysis. Specifically, functional Bahadur representations (FBR) in heterogeneous and homogeneous settings, i.e., Theorems 3.2 and 4.2, are proved based on the aforementioned technical tools, which can extract the leading terms from the estimators. Our FBR theory is a nontrivial extension of Shang (2010) and Shang and Cheng (2013) to panel data models, which plays a central role in our theoretical study.

The rest of this paper is structured as follows. Section 2 contains some technical preliminaries including an introduction to panel data models with IFE and an RKHS framework. Sections 3 and 4 contain the main results. Specifically, in heterogeneous setting, Section 3 includes estimation procedures for each individual parameters, and derives joint asymptotic normality for the estimators. Constructions of confidence interval and prediction interval are also mentioned. In homogeneous setting, Section 4 includes an estimation procedure for the common regression function and derives its marginal asymptotic normality. Section 5 examines the proposed methods based on a simulation study and real data analysis. Proofs of the main theorems are deferred to Section 6, and proofs of other results are separated as a supplement document.

\section{Preliminary}

\subsection{Panel Data Models with Interactive Fixed Effects}

Let $Y_{i t}$ be a real-valued observation and $X_{i t} \in \mathcal{X}_{i} \subseteq \mathbb{R}^{d}$ be a real vector of observed covariates, both collected on the $i$ th unit at time $t$, for $i \in[N]:=\{1,2, \ldots, N\}, t \in[T]:=\{1, \ldots, T\}$. Suppose that the observations follow a semiparametric regression model

$$
Y_{i t}=g_{i}\left(X_{i t}\right)+\gamma_{1 i}^{\prime} f_{1 t}+\gamma_{2 i}^{\prime} f_{2 t}+\epsilon_{i t}, \quad i \in[N], t \in[T]
$$

where $g_{i}$ is an unknown regression function, $f_{1 t} \in \mathbb{R}^{q_{1}}$ is a vector of observed common factors including intercept, $f_{2 t} \in \mathbb{R}^{q_{2}}$ is a vector of unobserved common factors, $\gamma_{1 i}$ and $\gamma_{2 i}$ are unobserved fixed vectors called as factor loadings, and $\epsilon_{i t}$ are unobserved random noise. The term $\gamma_{2 i}^{\prime} f_{2 t}$ is

called as interactive fixed effect. In general, we allow $g_{i}$ to be varying across the units, i.e., the panels demonstrate a heterogeneous structure. The special case $g_{i}=g$ for all $i \in[N]$ implies that the panels are homogeneous. In this paper, we will consider both cases from theoretical and methodological aspects. Since the factors in model (2.1) are not identifiable in the sense that they cannot be consistently estimated, further constraints are needed. Similar to Pesaran (2006), suppose that $X_{i t}$ are related to the factors through the following data generating equation:

$$
X_{i t}=\Gamma_{1 i}^{\prime} f_{1 t}+\Gamma_{2 i}^{\prime} f_{2 t}+v_{i t}, \quad i \in[N], t \in[T],
$$


where $\Gamma_{1 i} \in \mathbb{R}^{q_{1} \times d}$ and $\Gamma_{2 i} \in \mathbb{R}^{q_{2} \times d}$ are unobserved but fixed matrices, $v_{i t} \in \mathbb{R}^{d}$ is a vector of random noise. Other constraints to guarantee identifiability were proposed by Bai (2009) based on principle component analysis. Equation (2.2) provides a convenient way to remove the unobserved factor $f_{2 t}$ from model (2.1). Specifically, averaging (2.2) as done by Pesaran (2006), we get

$$
\bar{X}_{t}=\bar{\Gamma}_{1}^{\prime} f_{1 t}+\bar{\Gamma}_{2}^{\prime} f_{2 t}+\bar{v}_{t}, \quad t \in[T],
$$

where $\bar{X}_{t}=\frac{1}{N} \sum_{i=1}^{N} X_{i t}, \bar{\Gamma}_{1}=\frac{1}{N} \sum_{i=1}^{N} \Gamma_{1 i}, \bar{\Gamma}_{2}=\frac{1}{N} \sum_{i=1}^{N} \Gamma_{2 i}$, and $\bar{v}_{t}=\frac{1}{N} \sum_{i=1}^{N} v_{i t}$. Throughout we assume that $\bar{\Gamma}_{2} \bar{\Gamma}_{2}^{\prime}$ is invertible which may hold true if $q_{2} \leq d$. It follows from (2.3) that

$$
f_{2 t}=\left(\bar{\Gamma}_{2} \bar{\Gamma}_{2}^{\prime}\right)^{-1} \bar{\Gamma}_{2}\left(\bar{X}_{t}-\bar{\Gamma}_{1}^{\prime} f_{1 t}-\bar{v}_{t}\right)
$$

Replacing $f_{2 t}$ in (2.1) by (2.4), we get the following

$$
\begin{aligned}
Y_{i t} & =g_{i}\left(X_{i t}\right)+\gamma_{1 i}^{\prime} f_{1 t}+\gamma_{2 i}^{\prime}\left(\bar{\Gamma}_{2} \bar{\Gamma}_{2}^{\prime}\right)^{-1} \bar{\Gamma}_{2}\left(\bar{X}_{t}-\bar{\Gamma}_{1}^{\prime} f_{1 t}-\bar{v}_{t}\right)+\epsilon_{i t} \\
& =g_{i}\left(X_{i t}\right)+Z_{t}^{\prime} \beta_{i}+e_{i t}, \quad i \in[N], t \in[T],
\end{aligned}
$$

where $Z_{t}=\left(f_{1 t}^{\prime}, \bar{X}_{t}^{\prime}\right)^{\prime}, e_{i t}=\epsilon_{i t}-\gamma_{2 i}^{\prime}\left(\bar{\Gamma}_{2} \bar{\Gamma}_{2}^{\prime}\right)^{-1} \bar{\Gamma}_{2} \bar{v}_{t}$, and

$$
\beta_{i}=\left(\begin{array}{c}
\gamma_{1 i}-\bar{\Gamma}_{1} \bar{\Gamma}_{2}^{\prime}\left(\bar{\Gamma}_{2} \bar{\Gamma}_{2}^{\prime}\right)^{-1} \gamma_{2 i} \\
\bar{\Gamma}_{2}^{\prime}\left(\bar{\Gamma}_{2} \bar{\Gamma}_{2}^{\prime}\right)^{-1} \gamma_{2 i}
\end{array}\right)
$$

The equations (2.1), (2.2) and (2.5) play an important role in the proof of our main results.

\subsection{Kernel Ridge Regression}

Suppose $g_{i} \in \mathcal{H}_{i}$, where $\mathcal{H}_{i}$ is a Reproducing Kernel Hilbert Space (RKHS). Specifically, $\mathcal{H}_{i}$ is a Hilbert space of real-valued functions on $\mathcal{X}_{i}$, endowed with an inner product $\langle\cdot, \cdot\rangle_{\mathcal{H}_{i}}$, satisfying the property: for any $x \in \mathcal{X}_{i}$, there exists a unique element $\bar{K}_{x}^{(i)} \in \mathcal{H}_{i}$ such that for every $g \in \mathcal{H}_{i}$, $\left\langle\bar{K}_{x}^{(i)}, g\right\rangle_{\mathcal{H}_{i}}=g(x)$. The reproducing kernel function is defined by $\bar{K}^{(i)}\left(x_{1}, x_{2}\right)=\bar{K}_{x_{1}}^{(i)}\left(x_{2}\right)$, for any $x_{1}, x_{2} \in \mathcal{X}_{i}$. The kernel $\bar{K}^{(i)}$ is symmetric, i.e., $\bar{K}^{(i)}\left(x_{1}, x_{2}\right)=\bar{K}^{(i)}\left(x_{2}, x_{1}\right)$, and the matrix $\overline{\mathcal{K}}^{(i)}:=\left[\bar{K}\left(x_{i}, x_{i}\right)\right]_{i, j=1}^{n}$ is semi-positive definite for any $x_{1}, \ldots, x_{n} \in \mathcal{X}_{i}$. Berlinet and ThomasAgnan (2004) provides a nice introduction to RKHS.

By Mercer's theorem, $\bar{K}^{(i)}$ admits a spectral expansion:

$$
\bar{K}^{(i)}\left(x_{1}, x_{2}\right)=\sum_{\nu=1}^{\infty} \varphi_{\nu}^{(i)}\left(x_{1}\right) \varphi_{\nu}^{(i)}\left(x_{2}\right) / \rho_{\nu}^{(i)}, x_{1}, x_{2} \in \mathcal{X}_{i}
$$

where $0<\rho_{1}^{(i)} \leq \rho_{2}^{(i)} \leq \cdots$ are eigenvalues and $\varphi_{\nu}^{(i)}$ are eigenfunctions which form an $L^{2}\left(P_{X_{i}}\right)$ orthonormal basis with $X_{i} \equiv X_{i 1}$. This paper focuses on RKHS generated by the following kernels. For simplicity, we use $a_{n} \asymp b_{n}$ to represent $a_{n}=O\left(b_{n}\right)$ and $b_{n}=O\left(a_{n}\right)$.

Finite Rank Kernel (FRK): The kernel $\bar{K}^{(i)}$ is said to have rank $k>0$ if $\rho_{\nu}^{(i)}=\infty$ for $\nu>k$. For instance, the $(k-1)$-order polynomial kernel $\bar{K}^{(i)}\left(x_{1}, x_{2}\right)=\left(1+x_{1}^{\prime} x_{2}\right)^{k-1}$ for $x_{1}, x_{2} \in \mathbb{R}^{d}$ has rank $k$. Clearly, an FRK of rank $k$ corresponds to a parametric space of dimension $k$. 
Polynomially Diverging Kernel (PDK): The kernel $\bar{K}^{(i)}$ is said to be polynomially diverging of order $k>0$ if it has eigenvalues satisfying $\rho_{\nu}^{(i)} \asymp \nu^{2 k}$ for $\nu \geq 1$. For instance, the $k$-order Sobolev space is an RKHS with a kernel polynomially diverging of order $k$; see Wahba (1990).

Exponentially Diverging Kernel (EDK): The kernel $\bar{K}^{(i)}$ is said to be exponentially diverging of order $k>0$ if its eigenvalues satisfy $\rho_{\nu}^{(i)} \asymp \exp \left(b \nu^{k}\right)$ for $\nu \geq 1$, for a constant $b>0$. For instance, Gaussian kernel $\bar{K}^{(i)}\left(x_{1}, x_{2}\right)=\exp \left(-\left|x_{1}-x_{2}\right|^{2}\right)$ corresponds to $k=1$; see Lu et al. (2016).

The results of this paper can be applied to more complicated RKHS such as Additive RKHS, as described in Remark 4.2.

Let $\Theta_{i}:=\mathbb{R}^{q_{1}+d} \times \mathcal{H}_{i}$. We estimate $\theta=(\beta, g) \in \Theta_{i}$ via the following Kernel Ridge Regression:

$$
\begin{aligned}
\widehat{\theta}_{i}=\left(\widehat{\beta}_{i}, \widehat{g}_{i}\right) & =\arg \min _{\theta \in \Theta_{i}} \ell_{i, M, \eta_{i}}(\theta) \\
& \equiv \arg \min _{\theta \in \Theta_{i}}\left\{\frac{1}{2 T} \sum_{t=1}^{T}\left(Y_{i t}-g\left(X_{i t}\right)-Z_{t}^{\prime} \beta\right)^{2}+\frac{\eta_{i}}{2}\|g\|_{\mathcal{H}_{i}}^{2}\right\},
\end{aligned}
$$

where $M=(N, T)$ and $\eta_{i}>0$ is called as a regularization parameter.

Our results will rely on an RKHS structure on $\Theta_{i}$. Specifically, we will follow Cheng and Shang (2015) to construct two operators $R_{i}: \mathcal{U}_{i} \rightarrow \Theta_{i}$ and $P_{i}: \Theta_{i} \rightarrow \Theta_{i}$, where $\mathcal{U}_{i} \equiv\{u=(x, z): x \in$ $\left.\mathcal{X}_{i}, z \in \mathbb{R}^{q_{1}+d}\right\}$, such that for any $u=(x, z) \in \mathcal{U}_{i}$ and $\theta=(\beta, g) \in \Theta_{i}$, the following holds:

$$
\left\langle R_{i} u, \widetilde{\theta}\right\rangle_{i}=\widetilde{g}(x)+z^{\prime} \widetilde{\beta}, \quad\left\langle P_{i} \theta, \widetilde{\theta}\right\rangle_{i}=\eta_{i}\langle g, \widetilde{g}\rangle_{\mathcal{H}_{i}}, \quad \text { for any } \widetilde{\theta}=(\widetilde{\beta}, \widetilde{g}) \in \Theta_{i},
$$

where $\langle\cdot, \cdot\rangle_{i}$ is an inner product on $\Theta_{i}$ to be defined later in (2.9).

For any $x \in \mathcal{X}_{i}$, define $G_{i}(x)=E\left\{Z \mid X_{i}=x\right\}$ and $\Omega_{i}=E\left\{\left(Z-G_{i}\left(X_{i}\right)\right)\left(Z-G_{i}\left(X_{i}\right)\right)^{\prime}\right\}$, where $Z=Z_{1}$. Clearly, $\Omega_{i}$ is a square matrix of dimension $q_{1}+d$. In the below we require the eigenvalues of $\Omega_{i}$ to be bounded away from zero and infinity, a standard condition to guarantee semiparametric efficiency; see, e.g., Mammen and van de Geer (1997); Cheng and Shang (2015). Besides, $G_{i}$ are assumed to be $L^{2}$ integrable.

Assumption A1. For $i \in[N], G_{i} \in L^{2}\left(P_{X_{i}}\right)$. Furthermore, $c_{1} \leq \lambda_{\min }\left(\Omega_{i}\right) \leq \lambda_{\max }\left(\Omega_{i}\right) \leq c_{2}$ for positive constants $c_{1}, c_{2}$, where $\lambda_{\min }(\cdot)$ and $\lambda_{\max }(\cdot)$ are minimal and maximal eigenvalues.

For any $\theta_{k}=\left(\beta_{k}, g_{k}\right) \in \Theta_{i}, k=1,2$, define

$$
\left\langle\theta_{1}, \theta_{2}\right\rangle_{i}=E\left\{\left(g_{1}\left(X_{i}\right)+Z^{\prime} \beta_{1}\right)\left(g_{2}\left(X_{i}\right)+Z^{\prime} \beta_{2}\right)\right\}+\eta_{i}\left\langle g_{1}, g_{2}\right\rangle_{\mathcal{H}_{i}} .
$$

Define $\left\langle g_{1}, g_{2}\right\rangle_{\star, i}=\left\langle\left(0, g_{1}\right),\left(0, g_{2}\right)\right\rangle_{i}$. Following Cheng and Shang (2015), Assumption A1 implies that $\langle\cdot, \cdot\rangle_{i}$ and $\langle\cdot, \cdot\rangle_{\star, i}$ are valid inner products on $\Theta_{i}$ and $\mathcal{H}_{i}$, respectively. Meanwhile, $\left(\mathcal{H}_{i},\langle\cdot, \cdot\rangle_{\star, i}\right)$ is an RKHS with kernel $K^{(i)}(x, y) \equiv \sum_{\nu=1}^{\infty} \varphi_{\nu}^{(i)}(x) \varphi_{\nu}^{(i)}(y) /\left(1+\eta_{i} \rho_{\nu}^{(i)}\right), x, y \in \mathcal{X}_{i}$. We can further find a positive definite self-adjoint operator $W_{i}: \mathcal{H}_{i} \rightarrow \mathcal{H}_{i}$ and an element $A_{i} \in \mathcal{H}_{i}^{q_{1}+d}$ such that $\left\langle W_{i} g_{1}, g_{2}\right\rangle_{\star, i}=\eta_{i}\left\langle g_{1}, g_{2}\right\rangle_{\mathcal{H}_{i}},\left\langle A_{i}, g\right\rangle_{\star, i}=V_{i}\left(G_{i}, g\right)$, for any $g, g_{1}, g_{2} \in \mathcal{H}_{i}$, where $V_{i}\left(g_{1}, g_{2}\right)=$ $E\left\{g_{1}\left(X_{i}\right) g_{2}\left(X_{i}\right)\right\}$. Define $\Sigma_{i}=E\left\{G_{i}\left(X_{i}\right)\left(G_{i}\left(X_{i}\right)-A_{i}\left(X_{i}\right)\right)^{\prime}\right\}$, a symmetric matrix of dimension $q_{1}+d$. Following Cheng and Shang (2015), Proposition 2.1 below guarantees (2.8). 
Proposition 2.1. For any $u=(x, z) \in \mathcal{U}_{i}$ and for any $\theta=(\beta, g) \in \Theta_{i}$, (2.8) holds for $R_{i} u=$ $\left(H_{u}^{(i)}, T_{u}^{(i)}\right)$ and $P_{i} \theta=\left(H_{g}^{(\star i)}, T_{g}^{(\star i)}\right)$, where

$$
\begin{aligned}
H_{u}^{(i)} & =\left(\Omega_{i}+\Sigma_{i}\right)^{-1}\left(z-V_{i}\left(G_{i}, K_{x}^{(i)}\right)\right), \\
T_{u}^{(i)} & =K_{x}^{(i)}-A_{i}^{\prime}\left(\Omega_{i}+\Sigma_{i}\right)^{-1}\left(z-V_{i}\left(G_{i}, K_{x}^{(i)}\right)\right), \\
H_{g}^{(\star i)} & =-\left(\Omega_{i}+\Sigma_{i}\right)^{-1} V_{i}\left(G_{i}, W_{i} g\right), \\
T_{g}^{(\star i)} & =W_{i} g+A_{i}^{\prime}\left(\Omega_{i}+\Sigma_{i}\right)^{-1} V_{i}\left(G_{i}, W_{i} g\right) .
\end{aligned}
$$

A direct application of Proposition 2.1 is to exactly calculate the Fréchet derivatives of $\ell_{i, M, \eta_{i}}$. Define $U_{i t}=\left(X_{i t}, Z_{t}\right)$ for $i \in[N], t \in[T]$. For $\theta=(\beta, g), \Delta \theta=(\Delta \beta, \Delta g) \in \Theta_{i}$, we have

$$
\begin{aligned}
D \ell_{i, M, \eta_{i}}(\theta) \Delta \theta & =\left\langle-\frac{1}{T} \sum_{t=1}^{T}\left(Y_{i t}-\left\langle R_{i} U_{i t}, \theta\right\rangle_{i}\right) R_{i} U_{i t}+P_{i} \theta, \Delta \theta\right\rangle_{i} \equiv\left\langle S_{i, M, \eta_{i}}(\theta), \Delta \theta\right\rangle_{i}, \\
D S_{i, M, \eta_{i}}(\theta) \Delta \theta & =\frac{1}{T} \sum_{t=1}^{T}\left\langle R_{i} U_{i t}, \Delta \theta\right\rangle_{i} R_{i} U_{i t}+P_{i} \Delta \theta \\
D^{2} S_{i, M, \eta_{i}}(\theta) & =0 .
\end{aligned}
$$

\section{Heterogeneous Model}

In this section, we consider heterogeneous model (2.1) where the $g_{i}$ 's are assumed to be different across the units. We will estimate each $g_{i}$ through penalized estimations, and develop a joint asymptotic theory for the estimators. Our joint asymptotic results lead to novel statistical procedures as well as rediscover the existing marginal asymptotic results.

\subsection{Estimation Procedure}

By representer theorem (Wahba, 1990), the minimizer $\widehat{g}_{i}$ of $(2.7)$ has the expression

$$
g(x)=\sum_{t=1}^{T} a_{t} \bar{K}^{(i)}\left(X_{i t}, x\right)=a^{\prime} \bar{K}_{x}^{(i)}, \quad x \in \mathcal{X}_{i}
$$

where $a=\left(a_{1}, \cdots, a_{T}\right)^{\prime}$ and $\bar{K}_{x}^{(i)}=\left(\bar{K}^{(i)}\left(X_{i 1}, x\right), \cdots, \bar{K}^{(i)}\left(X_{i T}, x\right)\right)^{\prime}$. Based on (3.1) we have

$$
\|g\|_{\mathcal{H}_{i}}^{2}=\left\langle\sum_{t=1}^{T} a_{t} \bar{K}^{(i)}\left(X_{i t}, \cdot\right), \sum_{t=1}^{T} a_{t} \bar{K}^{(i)}\left(X_{i t}, \cdot\right)\right\rangle_{\mathcal{H}_{i}}=a^{\prime} \overline{\mathcal{K}}^{(i)} a
$$

where $\overline{\mathcal{K}}^{(i)}=\left(\bar{K}_{X_{i 1}}^{(i)}, \cdots, \bar{K}_{X_{i T}}^{(i)}\right) \in \mathbb{R}^{T \times T}$ is semi-positive definite. So, (2.7) can be equally transformed to the following:

$$
\left(\widehat{a}_{i}, \widehat{\beta}_{i}\right)=\arg \min _{a \in \mathbb{R}^{T}, \beta \in \mathbb{R}^{q_{1}+d}} \frac{1}{2 T}\left(Y_{i}-\overline{\mathcal{K}}^{(i)} a-Z \beta\right)^{\prime}\left(Y_{i}-\overline{\mathcal{K}}^{(i)} a-Z \beta\right)+\frac{\eta_{i}}{2} a^{\prime} \overline{\mathcal{K}}^{(i)} a,
$$


where $Y_{i}=\left(Y_{i 1}, \cdots, Y_{i T}\right)^{\prime}$ and $Z=\left(Z_{1}, \cdots, Z_{T}\right)^{\prime}$. The solution to (3.3) has expression

$$
\begin{aligned}
\widehat{a}_{i} & =\left(\left(I_{T}-Z\left(Z^{\prime} Z\right)^{-1} Z^{\prime}\right) \overline{\mathcal{K}}^{(i)}+T \eta_{i} I_{T}\right)^{-1}\left(I_{T}-Z\left(Z^{\prime} Z\right)^{-1} Z^{\prime}\right) Y_{i} \\
\widehat{\beta}_{i} & =\left(Z^{\prime} Z\right)^{-1} Z^{\prime}\left(Y_{i}-\overline{\mathcal{K}}^{(i)} \widehat{a}_{i}\right) .
\end{aligned}
$$

Then we estimate $g_{i}$ by $\widehat{g}_{i}(x)=\widehat{a}_{i}^{\prime} \bar{K}_{x}^{(i)}$ for any $x \in \mathcal{X}_{i}$.

Remark 3.1. In practice, we choose $\eta_{i}$ by minimizing the following GCV function:

$$
\widehat{\eta}_{i}=\arg \min _{\eta_{i}>0} G C V_{i}\left(\eta_{i}\right) \equiv \arg \min _{\eta_{i}>0} \frac{\left\|\left(I_{T}-B_{\eta_{i}}\right) Y_{i}\right\|_{2}^{2}}{T\left[1-\operatorname{tr}\left(B_{\eta_{i}}\right) /(T)\right]^{2}},
$$

where $B_{\eta_{i}}$ is the so-called smoothing matrix, i.e., a $T \times T$ matrix satisfying $\widehat{Y}_{i}=B_{\eta_{i}} Y_{i}$, where $\widehat{Y}_{i}=\overline{\mathcal{K}}^{(i)} \widehat{a}_{i}+Z \widehat{\beta}_{i}$ is the fitted response vector.

\subsection{Rate of Convergence}

We will derive the rate of convergence for $\widehat{\theta}_{i}$. Before that, let us assume some technical conditions. For $t \geq j \geq 1$, define $\mathcal{F}_{j}^{t}=\sigma\left(f_{1 l}, f_{2 l}: j \leq l \leq t\right)$. Define $\phi$-mixing coefficients

$$
\phi(t)=\sup _{t_{1} \geq 1} \sup _{\substack{A \in \mathcal{F}_{1}^{t_{1}} B \in \mathcal{F}_{t_{1}+t}^{\infty} \\ P(B)>0}}|P(A \mid B)-P(A)|, \quad t \geq 0 .
$$

Assumption A2. (a) $\left\{v_{i t}: i \in[N], t \in[T]\right\}$ are i.i.d., and $\left\{\epsilon_{i t}: i \in[N], t \in[T]\right\}$ are i.i.d., both of zero means. Furthermore, $v_{i t}$ 's and $\epsilon_{i t}$ 's are independent.

(b) Both $\left\{f_{1 t}: t \in[T]\right\}$ and $\left\{f_{2 t}: t \in[T]\right\}$ are strictly stationary process satisfying the following $\phi$-mixing condition: $\sum_{t=0}^{\infty} \phi(t)^{1-4 / \alpha}<\infty$, where $\alpha>4$ is a constant. $\left(f_{1 t}, f_{2 t}\right)$ is distributed independently of $v_{i t}$ 's and $\epsilon_{i t}$ 's.

(c) $E\left\{\left\|f_{1 t}\right\|_{2}^{\alpha}\right\}<\infty, E\left\{\left\|f_{1 t}\right\|_{2}^{\alpha}\right\}<\infty, E\left\{\left|v_{i t}\right|^{\alpha}\right\}<\infty, E\left\{\left|\epsilon_{i t}\right|^{\alpha}\right\}<\infty$, where $\|\cdot\|_{2}$ denotes the Euclidean norm.

(d) $\sup _{i \geq 1}\left\|\Delta_{i}\right\|_{2}<\infty$, where $\Delta_{i}=\gamma_{2 i}^{\prime}\left(\bar{\Gamma}_{2} \bar{\Gamma}_{2}^{\prime}\right)^{-1} \bar{\Gamma}_{2}$.

Assumption A2(a) requires that the variables $\varepsilon_{i t}, v_{i t}$ are zero-mean independent. Assumption A2(b) specify that the factors are strictly stationary and $\phi$-mixing, and independent of $v_{i t}$ 's and $\epsilon_{i t}$ 's. Independence assumption can be relaxed to mixing conditions with more tedious technical arguments. Assumption A2(c) requires that the variables have finite $\alpha$-moments. Assumption A2(d) requires that the vectors $\Delta_{i}$ based on "true" factor loadings are uniformly bounded.

The following assumption says that $\varphi_{\nu}^{(i)}$ are uniformly bounded and $\varphi_{\nu}^{(i)}, \rho_{\nu}^{(i)}$ simultaneously diagonalize $V_{i}$ and $\langle\cdot, \cdot\rangle_{\mathcal{H}_{i}}$, a standard assumption in kernel ridge regression literature, e.g., Shang and Cheng (2013). This condition holds for polynomially diverging kernels, exponentially diverging kernels, or finite rank kernels on compactly supported $\mathcal{X}_{i}$; see, e.g., Wahba (1990); Shang and Cheng (2013); Zhao et al. (2016). Besides, we need $1 \notin \mathcal{H}_{i}$ for identifiability. 
Assumption A3. For any $i \in[N], \sup _{\nu \geq 1} \sup _{x \in \mathcal{X}_{i}}\left|\varphi_{\nu}^{(i)}(x)\right|<\infty$ and

$$
V_{i}\left(\varphi_{\nu}^{(i)}, \varphi_{\mu}^{(i)}\right)=\delta_{\nu \mu}, \quad\left\langle\varphi_{\nu}^{(i)}, \varphi_{\mu}^{(i)}\right\rangle_{\mathcal{H}_{i}}=\rho_{\nu}^{(i)} \delta_{\nu \mu}, \quad \nu, \mu \geq 1
$$

where $\delta_{\nu \mu}$ is the Kronecker's delta. Furthermore, $1 \notin \mathcal{H}_{i}$, and any $g \in \mathcal{H}_{i}$ satisfies $g=\sum_{\nu=1}^{\infty} g_{\nu} \varphi_{\nu}^{(i)}$, where $g_{\nu}=V_{i}\left(g, \varphi_{\mu}^{(i)}\right)$ is a real sequence satisfying $\sum_{\nu=1}^{\infty} \rho_{\nu}^{(i)} g_{\nu}^{2}<\infty$.

For any $\theta=(\beta, g) \in \Theta_{i}$, define $\|\theta\|_{i, \text { sup }}=\sup _{x \in \mathcal{X}_{i}}|g(x)|+\|\beta\|_{2}$. For $p, \delta>0$, define $\mathcal{G}_{i}(p)=$ $\left\{\theta=(\beta, g) \in \Theta_{i}:\|\theta\|_{i, \text { sup }} \leq 1,\|g\|_{\mathcal{H}_{i}} \leq p\right\}$ and the corresponding entropy integral

$$
J_{i}(p, \delta)=\int_{0}^{\delta} \psi_{2}^{-1}\left(D_{i}\left(\varepsilon, \mathcal{G}_{i}(p),\|\cdot\|_{i, \mathrm{sup}}\right)\right) d \varepsilon+\delta \psi_{2}^{-1}\left(D_{i}\left(\delta, \mathcal{G}_{i}(p),\|\cdot\|_{i, \mathrm{sup}}\right)^{2}\right),
$$

where $\psi_{2}(s)=\exp \left(s^{2}\right)-1$ and $D_{i}\left(\varepsilon, \mathcal{G}_{i}(p),\|\cdot\|_{i \text {,sup }}\right)$ is the $\varepsilon$-packing number of $\mathcal{G}_{i}(p)$ in terms of $\|\cdot\|_{i \text {,sup }}$-metric. Let $\theta_{i 0}=\left(\beta_{i 0}, g_{i 0}\right)$ denote the "true" value of $(\beta, g)$ in $(2.5)$. Define

$$
h_{i}=\sum_{\nu=1}^{\infty}\left(1+\eta_{i} \rho_{\nu}^{(i)}\right)^{-1}, \quad r_{i, M}=\left(T h_{i}\right)^{-1 / 2}+\eta_{i}^{1 / 2}+\left(N h_{i}\right)^{-1 / 2} .
$$

It can be shown that $h_{i} \asymp \eta_{i}^{1 /(2 k)}$ for $k$-order PDK; $h_{i} \asymp\left(\log \left(1 / \eta_{i}\right)\right)^{-1 / k}$ for $k$-order EDK. We use $(N, T) \rightarrow \infty$ to represent both $N \rightarrow \infty$ and $T \rightarrow \infty$.

Theorem 3.1. Suppose that Assumptions A1-A3 are satisfied. Furthermore, as $(N, T) \rightarrow \infty$, the following conditions hold:

$$
\begin{aligned}
& \eta_{i}=o(1), \quad h_{i}=o(1), \quad T^{2 / \alpha-1} h_{i}^{-1}=o(1), \quad \eta_{i}+\frac{1}{N h_{i}}=O\left(h_{i}\right), \\
& T^{-1 / 2+1 / \alpha} h_{i}^{-1 / 2} \max \left\{h_{i}^{-1 / 2}, T^{1 / \alpha}\right\} J_{i}\left(\left(\eta_{i}^{-1} h_{i}\right)^{1 / 2}, 1\right) \\
& \times \sqrt{\log N+\log \log \left(T J_{i}\left(\left(\eta_{i}^{-1} h_{i}\right)^{1 / 2}, 1\right)\right)}=o(1) .
\end{aligned}
$$

Then for any $i \in[N],\left\|\widehat{\theta}_{i}-\theta_{i 0}\right\|_{i}=O_{P}\left(r_{i, M}\right)$, as $(N, T) \rightarrow \infty$.

Theorem 3.1 presents a rate of convergence for $\widehat{\theta}_{i}$ in $\|\cdot\|_{i}$-norm which relies on $h_{i}, N, T$. The $\|\cdot\|_{i}$-norm is stronger than the commonly used $L^{2}$-norm in literature; see $\mathrm{Su}$ and Jin (2012), Su and Zhang (2013). The optimal choice of $h_{i}$, denoted $h_{i}^{\star}$, relies on the type of kernels and the relationship of $N, T$, i.e., $N \geq T$ or $N<T$. The optimal convergence rate, denoted $r_{i, M}^{\star}$, can be calculated accordingly; see Table 1 . We observe that $h_{i}^{\star}, r_{i, M}^{\star}$ only depend on the smaller value of $N, T$ in both PDK and EDK. Rate conditions (3.5) are satisfied under $h_{i} \asymp h_{i}^{\star}$.

\begin{tabular}{ccccc} 
& \multicolumn{2}{c}{$N \geq T$} & \multicolumn{2}{c}{$N<T$} \\
\cline { 2 - 5 } & PDK & EDK & PDK & EDK \\
\hline$h_{i}^{\star}$ & $T^{-1 /(2 k+1)}$ & $(\log T)^{-1 / k}$ & $N^{-1 /(2 k+1)}$ & $(\log N)^{-1 / k}$ \\
$r_{i, M}^{\star}$ & $T^{-k /(2 k+1)}$ & $T^{-1 / 2}(\log T)^{1 /(2 k)}$ & $N^{-k /(2 k+1)}$ & $N^{-1 / 2}(\log N)^{1 /(2 k)}$ \\
\hline \multicolumn{4}{c}{ TABLE 1} &
\end{tabular}

A summary of $h_{i}^{\star}$ and $r_{i}^{\star}$ in $k$-order PDK and $k$-order EDK. 


\subsection{Joint Asymptotic Distribution}

The aim of this section is to derive joint asymptotic normality for $\widehat{\theta}_{i}=\left(\widehat{\beta}_{i}, \widehat{g}_{i}\right)$ which is new in literature. Our result naturally leads to marginal asymptotic normality obtained by $\mathrm{Su}$ and Jin (2012). More importantly, our joint asymptotic result can be used to construct confidence interval for regression mean and prediction interval for future response variable. As far as we know, these are the first asymptotic valid intervals for prediction purposes. Before proceeding further, we give a technical result, called as Functional Bahadur Representation (FBR), to characterize the leading term of the estimator.

Theorem 3.2 (FBR for Heterogeneous Model). Suppose that Assumptions A1-A3 are satisfied. Then we have

$$
\left\|\widehat{\theta}_{i}-\theta_{i 0}+S_{i, M, \eta_{i}}\left(\theta_{i 0}\right)\right\|_{i}=O_{P}\left(a_{M}\right), \quad \text { as }(N, T) \rightarrow \infty,
$$

where $a_{M}=r_{i, M} h_{i}^{-1 / 2} T^{1 / \alpha-1 / 2}\left(h_{i}^{-1 / 2}+T^{1 / \alpha}\right) J_{i}\left(p_{i}, 1\right) \sqrt{\log N+\log \log \left(T J_{i}\left(p_{i}, 1\right)\right)}+h_{i}^{-1 / 2} T^{2 / \alpha-1}$ and $p_{i}=\left(\eta_{i}^{-1} h_{i}\right)^{1 / 2}$.

Theorem 3.2 provides a higher order approximation for $\widehat{\theta}_{i}-\theta_{i 0}$ with leading term $S_{i, M, \eta_{i}}\left(\theta_{i 0}\right)$, which generalizes Shang (2010) and Shang and Cheng (2013) to panel data settings. The rate of the remainder term $a_{M}$ can be shown to be $O\left(N^{-1 / 2}\right)$ or $O\left(T^{-1 / 2}\right)$ if we choose $h_{i} \asymp h_{i}^{\star}$, where the values of $h_{i}^{\star}$ are summarized in Table 1 . Thus, the remainder term is asymptotically negligible compared to $S_{i, M, \eta_{i}}\left(\theta_{i 0}\right)$. This lemma can be used to prove the following joint asymptotic normality for $\widehat{\theta}_{i}$. The proof relies on a central limit theorem on $S_{i, M, \eta_{i}}\left(\theta_{i 0}\right)$. Let $\theta_{i 0}^{\star}=\left(\beta_{i 0}^{\star}, g_{i 0}^{\star}\right) \equiv\left(i d-P_{i}\right) \theta_{i 0}$.

Theorem 3.3. Suppose that Assumptions A1-A3 are all satisfied. Furthermore, $h_{i}=o(1)$, $\left(N h_{i}\right)^{-1}=o(1), a_{M}=o\left(T^{-1 / 2} h_{i}^{1 / 2}\right)$, and for $x_{0} \in \mathcal{X}_{i}, h_{i} V_{i}\left(K_{x_{0}}^{(i)}, K_{x_{0}}^{(i)}\right) \rightarrow \sigma_{x_{0}}^{2}, h_{i}^{1 / 2}\left(W_{i} A_{i}\right)\left(x_{0}\right) \rightarrow$ $\alpha_{x_{0}}$, and $h_{i}^{1 / 2} A_{i}\left(x_{0}\right) \rightarrow-\beta_{x_{0}}$, where $\sigma_{x_{0}}^{2}>0, \alpha_{x_{0}}, \beta_{x_{0}} \in \mathbb{R}^{q_{1}+d}$ are nonrandom constants. Then,

$$
\left(\begin{array}{c}
\sqrt{T}\left(\widehat{\beta}_{i}-\beta_{i 0}^{\star}\right)-\sqrt{T} E\left\{e_{i t} H_{U_{i t}}^{(i)}\right\} \\
\sqrt{T h_{i}}\left(\widehat{g}_{i}\left(x_{0}\right)-g_{i 0}^{\star}\left(x_{0}\right)\right)-\sqrt{T h_{i}} E\left\{e_{i t} T_{U_{i t}}^{(i)}\left(x_{0}\right)\right\}
\end{array}\right) \stackrel{d}{\rightarrow} N\left(0, \Psi^{\star}\right), \quad \text { as }(N, T) \rightarrow \infty,
$$

where

$$
\Psi^{\star}=\sigma_{\epsilon}^{2}\left(\begin{array}{cc}
\Omega_{i}^{-1} & \Omega_{i}^{-1}\left(\alpha_{x_{0}}+\beta_{x_{0}}\right) \\
\left(\alpha_{x_{0}}+\beta_{x_{0}}\right)^{\prime} \Omega_{i}^{-1} & \sigma_{x_{0}}^{2}+2 \beta_{x_{0}}^{\prime} \Omega_{i}^{-1} \alpha_{x_{0}}+\beta_{x_{0}}^{\prime} \Omega_{i}^{-1} \beta_{x_{0}}
\end{array}\right) \text {, and } \sigma_{\epsilon}^{2}=\operatorname{Var}\left(\epsilon_{i t}\right) .
$$

Theorem 3.3 proves joint asymptotic normality for $\widehat{\beta}_{i}$ and $\widehat{g}_{i}\left(x_{0}\right)$. The estimators are nonetheless not (asymptotically) unbiased, i.e., they do not converge to the truth $\beta_{i 0}$ and $g_{i 0}\left(x_{0}\right)$. To correct the bias, we need to assume $T / N=o(1)$ as in the following Theorem 3.4. This condition means that the number of observations within each individual unit is strictly smaller than the number of units, which can provide more cross section information. We expect that the bias cannot be corrected if $T \geq N$. Indeed, our theoretical analysis indicates a possibly sharp upper bound $\sqrt{T} E\left\{e_{i t} H_{U_{i t}}^{(i)}\right\}=$ $O_{P}(\sqrt{T / N})$. When $T \geq N$, this term will result in uncorrectable bias in estimating $\beta_{i}$. Relevant 
assumptions exist in literature for bias correction, e.g., $T / N^{2}=o(1)$ considered by Pesaran (2006) in parametric setting; $\kappa T / N=o(1)$ considered by $\mathrm{Su}$ and Jin (2012) in sieve estimation, where $\kappa$ represents the discrete truncation parameter (or, number of basis functions). Compared to the latter, our condition is weaker. Another condition for bias correction is that $G_{i}$ is sufficiently smooth, i.e., condition (3.9) in Theorem 3.4. Such condition holds if the conditional distribution of the factor variable $f_{1 t}$ given $X_{i t}$ is smooth. As a by-product, $\widehat{\beta}_{i}$ and $\widehat{g}_{i}\left(x_{0}\right)$ become asymptotically independent which facilitates the applications, e.g., one does not need to estimate the correlation between the two estimators. Define $G_{i}=\left(G_{i, 1}, \ldots, G_{i, q_{1}+d}\right)^{\prime}$.

Theorem 3.4. Suppose that the conditions in Theorem 3.3 hold, $T \eta_{i}=o(1)$ and $T / N=o(1)$. Furthermore, there exists a positive non-decreasing sequence $k_{\nu}$ with $\sum_{\nu \geq 1} 1 / k_{\nu}<\infty$ such that

$$
\sum_{\nu}\left|V_{i}\left(G_{i, k}, \varphi_{\nu}^{(i)}\right)\right|^{2} k_{\nu}<\infty, \quad \text { for } k=1, \ldots, q_{1}+d
$$

Then we have, for any $x_{0} \in \mathcal{X}_{i}$,

$$
\left(\begin{array}{c}
\sqrt{T}\left(\widehat{\beta}_{i}-\beta_{i 0}\right) \\
\sqrt{T h_{i}}\left(\widehat{g}_{i}\left(x_{0}\right)-g_{i 0}\left(x_{0}\right)\right)
\end{array}\right) \stackrel{d}{\rightarrow} N(0, \Psi), \quad \text { as }(N, T) \rightarrow \infty,
$$

where

$$
\Psi=\sigma_{\epsilon}^{2}\left(\begin{array}{cc}
\Omega_{i}^{-1} & 0 \\
0 & \sigma_{x_{0}}^{2}
\end{array}\right)
$$

An application of Theorem 3.4 is the construction of confidence interval for regression mean. Suppose $X_{i t+1}=x_{i 0}$ and $f_{1 t+1}=f_{10}$ with known $x_{i 0}$ and $f_{10}$, i.e., the predictor variables of each individual are observed at future time $t+1$. By (2.5), the conditional mean of $Y_{i t+1}$ is $\mu_{i 0} \equiv E\left\{Y_{i t+1} \mid X_{i t+1}=x_{i 0}, f_{1 t+1}=f_{10}\right\} \approx g_{i 0}\left(x_{i 0}\right)+z_{0}^{\prime} \beta_{i 0}$, where $z_{0}=\left(f_{10}^{\prime}, N^{-1} \sum_{i=1}^{N} x_{i 0}^{\prime}\right)^{\prime}$. We propose the following $1-\alpha$ confidence interval for $\mu_{i 0}$ :

$$
\widehat{\mu}_{i} \pm z_{1-\alpha / 2} \frac{\sigma_{x_{0}} \sigma_{\epsilon}}{\sqrt{T h_{i}}}, \quad \text { where } \widehat{\mu}_{i}=\widehat{g}_{i}\left(x_{i 0}\right)+z_{0}^{\prime} \widehat{\beta}_{i} .
$$

Here, $z_{1-\alpha / 2}$ is the $(1-\alpha / 2)$-percentile of standard normal distribution. The following Corollary 3.5 guarantees the asymptotic validity of (3.12).

Corollary 3.5. Under the conditions of Theorem 3.4, we have, as $(N, T) \rightarrow \infty$,

$$
\sqrt{T h_{i}}\left(\widehat{\mu}_{i}-\mu_{i 0}\right) \stackrel{d}{\rightarrow} N\left(0, \sigma_{x_{0}}^{2} \sigma_{\epsilon}^{2}\right) .
$$

Another application of Theorem 3.4 is to construct the prediction interval for $Y_{i t+1}$. By (2.5),

$$
Y_{i t+1}-\widehat{\mu}_{i}=\left(\mu_{i 0}-\widehat{\mu}_{i}\right)+\epsilon_{i t+1}-\gamma_{2 i}^{\prime}\left(\bar{\Gamma}_{2} \bar{\Gamma}_{2}^{\prime}\right)^{-1} \bar{\Gamma}_{2} \bar{v}_{t+1}
$$

The proof of Theorem 3.1 indicates that the last term of (3.14) is $O_{P}\left(N^{-1 / 2}\right)$, whereas the first term is $O_{P}\left(\left(T h_{i}\right)^{-1 / 2}\right)$ thanks to Corollary 3.5. If $T h_{i}=o(N)$, i.e., the last term of (3.14) is 
asymptotically negligible, then the asymptotic distribution of (3.14) is a convolution of $F_{\epsilon}$ and the distribution of $N\left(0, \sigma_{x_{0}}^{2} \sigma_{\epsilon}^{2} /\left(T h_{i}\right)\right)$, where $F_{\epsilon}$ is the c.d.f. of $\epsilon_{i t+1}$. Let $q_{\alpha / 2}$ and $q_{1-\alpha / 2}$ be the $\alpha / 2$ - and $(1-\alpha / 2)$-percentiles of the convolution, then a $1-\alpha$ prediction interval for $Y_{i t+1}$ is

$$
\left[\widehat{\mu}_{i}+q_{\alpha / 2}, \widehat{\mu}_{i}+q_{1-\alpha / 2}\right]
$$

In particular, if $\epsilon_{i t+1} \sim N\left(0, \sigma_{\epsilon}^{2}\right)$, then (3.15) becomes the following

$$
\widehat{\mu}_{i} \pm z_{1-\alpha / 2} \sigma_{\epsilon} \sqrt{\sigma_{x_{0}}^{2} /\left(T h_{i}\right)+1} .
$$

The asymptotic variance $\sigma_{x_{0}}^{2}$ has an explicit expression

$$
\sigma_{x_{0}}^{2}=h_{i} V_{i}\left(K_{x_{0}}^{(i)}, K_{x_{0}}^{(i)}\right)=\sum_{\nu \geq 1} \frac{h_{i}\left|\varphi_{\nu}^{(i)}\left(x_{0}\right)\right|^{2}}{\left(1+\eta_{i} \rho_{\nu}^{(i)}\right)^{2}} .
$$

In practice, we can estimate $\sigma_{x_{0}}^{2}$ by replacing $\varphi_{\nu}^{(i)}$ and $\rho_{\nu}^{(i)}$ with their empirical counterparts such as kernel eigenvalues and kernel eigenfunctions; see, e.g., Braun (2006). Meanwhile, we estimate $\sigma_{\epsilon}^{2}$ by the following

$$
\widehat{\sigma}_{\epsilon}^{2}=\sum_{t=1}^{T}\left\{Y_{i t}-\widehat{g}_{i}\left(X_{i t}\right)-Z_{t}^{\prime} \widehat{\beta}_{i}\right\}^{2} / T .
$$

The following result shows that (3.16) is a consistent estimator.

Proposition 3.6. Under conditions of Theorem 3.1, if $r_{i, M}=o_{P}\left(h_{i}^{1 / 2}\right)$ and $r_{i, M}=o_{P}\left(T^{-1 / \alpha}\right)$, then $\widehat{\sigma}_{\epsilon}^{2} \rightarrow \sigma_{\epsilon}^{2}$ in probability, as $(N, T) \rightarrow \infty$.

\section{Homogeneous Model}

In this section, we consider a homogeneous case, i.e., $g_{i}=g$ for all $i \in[N]$. Assuming homogeneity, model (2.1) becomes the following

$$
Y_{i t}=g\left(X_{i t}\right)+\gamma_{1 i}^{\prime} f_{1 t}+\gamma_{2 i}^{\prime} f_{2 t}+\epsilon_{i t}, i \in[N], t \in[T]
$$

By (2.2) and a similar statement as (2.5), we can rewrite (4.1) as the following

$$
Y_{i t}=g\left(X_{i t}\right)+Z_{t}^{\prime} \beta_{i}+e_{i t}, \quad i \in[N], t \in[T]
$$

where $Z_{t}, \beta_{i}$ and $e_{i t}$ are given in (2.5).

We will provide a procedure for estimating $g$ and explore its asymptotic property. Our theoretical results hold when $M \rightarrow \infty$. Here $M \rightarrow \infty$ means either $(N, T) \rightarrow \infty$ or $N \rightarrow \infty$, fixed $T$. Whereas the estimation of $\beta_{i}$ is inconsistent when $T$ is fixed due to insufficient data in each individual unit. The $\beta_{i}$ will be treated as nuisance parameters throughout the whole section. 


\subsection{Estimation Procedure}

Suppose $g$ belongs to an RKHS $\mathcal{H} \subset L^{2}(\mathcal{X})$ with inner product $\langle\cdot, \cdot\rangle_{\mathcal{H}}$ and kernel $\bar{K}(\cdot, \cdot)$, where $\mathcal{X} \subseteq \mathbb{R}^{d}$. Our estimation is based on profile least squares:

Step (a). For any $g \in \mathcal{H}$, we estimate $\beta_{i}$ through the following

$$
\min _{\beta_{i} \in \mathbb{R}^{q_{1}+d}} \sum_{t=1}^{T}\left(Y_{i t}-g\left(X_{i t}\right)-Z_{t}^{\prime} \beta_{i}\right)^{2}=\min _{\beta_{i} \in \mathbb{R}^{q_{1}+d}}\left(Y_{i}-\tau_{i} g-\Sigma^{\prime} \beta_{i}\right)^{\prime}\left(Y_{i}-\tau_{i} g-\Sigma^{\prime} \beta_{i}\right), i \in[N],
$$

where $Y_{i}=\left(Y_{i 1}, \ldots, Y_{i T}\right)^{\prime}, \tau_{i} g=\left(g\left(X_{i 1}\right), \ldots, g\left(X_{i T}\right)\right)^{\prime}$ and $\Sigma=\left(Z_{1}, \ldots, Z_{T}\right)$. Recall that $\Sigma$ is $\left(q_{1}+\right.$ $d) \times T$. Suppose $T \geq q_{1}+d$ so that $\left(\Sigma \Sigma^{\prime}\right)^{-1}$ exists. Then (4.3) has solution $\widehat{\beta}_{i}=\left(\Sigma \Sigma^{\prime}\right)^{-1} \Sigma\left(Y_{i}-\tau_{i} g\right)$.

Step (b). Plug the above $\widehat{\beta}_{i}$ into (4.3). The minimum value of (4.3) is equal to $\left(Y_{i}-\tau_{i} g\right)^{\prime}\left(I_{T}-\right.$ $\left.\Sigma^{\prime}\left(\Sigma \Sigma^{\prime}\right)^{-1} \Sigma\right)\left(Y_{i}-\tau_{i} g\right)$. Then we estimate $g$ by the following

$$
\widehat{g}=\arg \min _{g \in \mathcal{H}} \ell_{M, \eta}(g) \equiv \arg \min _{g \in \mathcal{H}}\left\{\frac{1}{2 N T} \sum_{i=1}^{N}\left(Y_{i}-\tau_{i} g\right)^{\prime} P\left(Y_{i}-\tau_{i} g\right)+\frac{\eta}{2}\|g\|_{\mathcal{H}}^{2}\right\},
$$

where $\eta>0$ is a penalty parameter and $P=I_{T}-\Sigma^{\prime}\left(\Sigma \Sigma^{\prime}\right)^{-1} \Sigma$ with $I_{T}$ the $T \times T$ identity.

The above Step (b) yields an explicit solution. Specifically, by representer theorem, $\widehat{g}$ satisfies

$$
g(x)=\sum_{i=1}^{N} \sum_{t=1}^{T} a_{i t} \bar{K}\left(X_{i t}, x\right)=a^{\prime} \bar{K}_{x}
$$

where $a=\left(a_{11}, \cdots, a_{1 T}, \cdots, a_{N 1}, \cdots, a_{N T}\right)^{\prime}$ are constant scalars, and

$$
\bar{K}_{x}=\left(\bar{K}\left(X_{11}, x\right), \cdots, \bar{K}\left(X_{1 T}, x\right), \cdots, \bar{K}\left(X_{N 1}, x\right), \cdots, \bar{K}\left(X_{N T}, x\right)\right)^{\prime} .
$$

Similar to (3.2), $\|g\|_{\mathcal{H}}^{2}=a^{\prime} \overline{\mathcal{K}} a$, where $\overline{\mathcal{K}}=\left(\bar{K}_{X_{11}}, \cdots, \bar{K}_{X_{1 T}}, \cdots, \bar{K}_{X_{N 1}}, \cdots, \bar{K}_{X_{N T}}\right) \in \mathbb{R}^{N T \times N T}$. Therefore, we can rewrite $\ell_{M, \eta}(g)$ as $\ell_{M, \eta}(g)=(Y-\overline{\mathcal{K}} a)^{\prime} P_{N}(Y-\overline{\mathcal{K}} a) /(2 N T)+\eta a^{\prime} \overline{\mathcal{K}} a / 2$, where $Y=\left(Y_{11}, \cdots, Y_{1 T}, \cdots, Y_{N 1}, \cdots, Y_{N T}\right)^{\prime}$ is an $N T$-vector and $P_{N}=I_{N} \otimes P$ is $N T \times N T$. The minimizer $\widehat{a}$ has an expression $\widehat{a}=\left(P_{N} \overline{\mathcal{K}}+N T \eta I_{N T}\right)^{-1} P_{N} Y$. Then $\widehat{g}(x)=\widehat{a}^{\prime} \bar{K}_{x}$ for any $x \in \mathcal{X}$.

Remark 4.1. We propose the following GCV method for choosing $\eta$ in the above estimation:

$$
\widehat{\eta}=\arg \min _{\eta>0} G C V(\eta) \equiv \arg \min _{\eta>0} \frac{\left\|\left(I_{N T}-B_{\eta}\right) Y\right\|_{2}^{2}}{N T\left[1-\operatorname{tr}\left(B_{\eta}\right) /(N T)\right]^{2}},
$$

where $B_{\eta}$ is the $N T \times N T$ smoothing matrix defined similar to Remark 3.1.

\subsection{Rate of Convergence}

To derive the rate of convergence for $\widehat{g}$, let us adapt the framework of Section 3 to the homogeneous setting. Define $V(g, \widetilde{g})=\sum_{i=1}^{N} E\left\{\left(\tau_{i} g\right)^{\prime} P\left(\tau_{i} \widetilde{g}\right) \mid \mathcal{F}_{1}^{T}\right\} /(N T)$ for any $g, \widetilde{g} \in \mathcal{H}$. Suppose that $V(\cdot, \cdot)$ and $\langle\cdot, \cdot\rangle_{\mathcal{H}}$ are simultaneously diagonalizable. 
Assumption A4. There exist eigenfunctions $\varphi_{\nu} \in \mathcal{H}$ and a nondecreasing positive sequence of eigenvalues $\rho_{\nu}$ such that $V\left(\varphi_{\nu}, \varphi_{\mu}\right)=\delta_{\nu \mu},\left\langle\varphi_{\nu}, \varphi_{\mu}\right\rangle_{\mathcal{H}}=\rho_{\nu} \delta_{\nu \mu}$, for any $\nu, \mu \geq 1$. Furthermore, $1 \notin \mathcal{H}$ and any function $g \in \mathcal{H}$ admits a generalized Fourier expansion $g=\sum_{\nu \geq 1} V\left(g, \varphi_{\nu}\right) \varphi_{\nu}$. Both $\varphi_{\nu}$ and $\rho_{\nu}$ are $\mathcal{F}_{1}^{T}$-measurable and $c_{\varphi} \equiv \sup _{\nu \geq 1}\left\|\varphi_{\nu}\right\|_{\text {sup }}=O_{P}(1)$, i.e., $\varphi_{\nu}$ are stochastic uniformly bounded.

A4 type conditions are commonly used in literature to derive the rate of convergence for smoothing splines or kernel ridge regression; see Gu and Qiu (1993); Shang and Cheng (2013); Cheng and Shang (2015); Zhao et al. (2016). Classic ways to verify such conditions rely on variational methods; see Weinberger (1974). Nevertheless, Assumption A4 differs from literature in that the functional $V$ and the eigenpairs $\left(\rho_{\nu}, \varphi_{\nu}\right)$ are random. Fortunately, we can still verify Assumption A4 by adapting the classic variational method to this new setting. The exact verification is deferred to Lemma S.2 in appendix.

Define $\Sigma_{\star}=\left(Z_{1}^{\star}, \ldots, Z_{T}^{\star}\right)$, a square matrix of dimension $q_{1}+d$, where $Z_{t}^{\star}=\left(f_{1 t}^{\prime},\left(\bar{X}_{t}^{\star}\right)^{\prime}\right)^{\prime}$ and $\bar{X}_{t}^{\star}=\bar{X}_{t}-\bar{v}_{t}$ for $t \in[T]$. By (2.3) and Assumption A2, $\Sigma_{\star}$ is independent of the variables $v_{i t}$. Hence, $\Sigma_{\star}$ can be viewed as a "noiseless" analogy of $\Sigma$. In the below we impose a moment condition on the spectral norms of various matrices.

Assumption A5. There exist constants $\zeta>4$ and $c>0$ such that

$$
\begin{aligned}
& E\left(\left\|\left(\Sigma_{\star} \Sigma_{\star}^{\prime} / T\right)^{-1}\right\|_{o p}^{\zeta}\right) \leq c, \quad E\left(\left\|\left(\Sigma \Sigma^{\prime} / T\right)^{-1}\right\|_{o p}^{\zeta}\right) \leq c \text { and } \\
& E\left(\left\|\Sigma_{\star} \Sigma_{\star}^{\prime} / T\right\|_{o p}^{2 \zeta /(\zeta-4)}\right) \leq c, \quad E\left(\left\|\sum_{t=1}^{T} f_{2 t} f_{2 t}^{\prime} / T\right\|_{o p}^{2 \zeta /(\zeta-4)}\right) \leq c,
\end{aligned}
$$

where $\|\cdot\|_{\text {op }}$ represents the operator norm of square matrices.

For any $g, \widetilde{g} \in \mathcal{H}$, define $\langle g, \widetilde{g}\rangle=V(g, \widetilde{g})+\eta\langle g, \widetilde{g}\rangle_{\mathcal{H}}$. Following Cheng and Shang $(2015),(\mathcal{H},\langle\cdot, \cdot\rangle)$ is an RKHS with reproducing kernel denoted $K$. For convenience, define $\mathbb{X}_{i}=\left(X_{i 1}, \ldots, X_{i T}\right)^{\prime}$ and $K_{\mathbb{X}_{i}}=\left(K_{X_{i 1}}, \ldots, K_{X_{i T}}\right)^{\prime}$ for $i \in[N]$. Similar to Section 2.2, there exists a positive definite self-adjoint operator $W_{\eta}: \mathcal{H} \rightarrow \mathcal{H}$ such that $\left\langle W_{\eta} g, \widetilde{g}\right\rangle=\eta\langle g, \widetilde{g}\rangle_{\mathcal{H}}, g, \widetilde{g} \in \mathcal{H}$. Then the Fréchet derivatives of $\ell_{M, \eta}(g)$ have the following expressions

$$
\begin{aligned}
D \ell_{M, \eta}(g) \Delta \theta & =\left\langle-\frac{1}{N T} \sum_{i=1}^{N}\left(Y_{i}-\left\langle K_{\mathbb{X}_{i}}, g\right\rangle\right)^{\prime} P K_{\mathbb{X}_{i}}+W_{\eta} g, \Delta g\right\rangle \equiv\left\langle S_{M, \eta}(g), \Delta g\right\rangle, \\
D S_{M, \eta}(g) \Delta g & =\frac{1}{N T} \sum_{i=1}^{N}\left\langle K_{\mathbb{X}_{i}}, \Delta g\right\rangle^{\prime} P K_{\mathbb{X}_{i}}+W_{\eta} \Delta g, \\
D^{2} S_{M, \eta}(g) & =0 .
\end{aligned}
$$

For $p, \delta>0$, define $\mathcal{G}(p)=\left\{g \in \mathcal{H}:\|g\|_{\text {sup }} \leq 1,\|g\|_{\mathcal{H}} \leq p\right\}$ and an entropy integral

$$
J(p, \delta)=\int_{0}^{\delta} \psi_{2}^{-1}\left(D\left(\varepsilon, \mathcal{G}(p),\|\cdot\|_{\text {sup }}\right)\right) d \varepsilon+\delta \psi_{2}^{-1}\left(D\left(\delta, \mathcal{G}(p),\|\cdot\|_{\text {sup }}\right)^{2}\right)
$$


where recall that $D\left(\varepsilon, \mathcal{G}(p),\|\cdot\|_{\text {sup }}\right)$ is the $\varepsilon$-packing number of $\mathcal{G}(p)$ in terms of $\|\cdot\|_{\text {sup }}$-metric. Define

$$
h=\left(\sum_{\nu \geq 1} \frac{1}{1+\eta \rho_{\nu}}\right)^{-1}, \quad b_{N, p}=\sqrt{\log \log (N J(p, 1))} J(p, 1), \quad p=\left(c_{\varphi} \sqrt{h^{-1} \eta}\right)^{-1} .
$$

Theorem 4.1. Suppose that Assumptions A2, A4 and A5 are satisfied. Furthermore, $b_{N, p}=$ $o_{P}\left(N^{1 / 2} h\right)$. Then, as $M \rightarrow \infty,\left\|\widehat{g}-g_{0}\right\|=O_{P}\left(r_{M}\right)$, where $r_{M}=(N T h)^{-1 / 2}+\left(N h^{1 / 2}\right)^{-1}+\eta^{1 / 2}$.

Theorem 4.1 provides a rate of convergence for $\widehat{g}$. Like in Theorem 3.1, to yield optimal rate of convergence (denoted $r_{M}^{\star}$ ), the optimal choice of $h$ (denoted $h^{\star}$ ) relies on kernels and relationship of $N, T$. The following Table 2 summarizes the values of $h^{\star}$ and $r_{M}^{\star}$ in PDK and EDK. Interestingly, when $N \geq T, r_{M}^{\star}$ depends on $N T$; whereas $N<T, r_{M}^{\star}$ only depends on $N$. The latter implies that, when $N<T$, increasing time points will not change convergence rate. Moreover, it can be examined that the condition $b_{N, p}=o_{P}\left(N^{1 / 2} h\right)$ in Theorem 4.1 holds true when $h \asymp h^{\star}$.

\begin{tabular}{ccccc} 
& \multicolumn{2}{c}{$N \geq T$} & \multicolumn{2}{c}{$N<T$} \\
\cline { 2 - 5 } & PDK & EDK & PDK & EDK \\
\hline$h^{\star}$ & $(N T)^{-1 /(2 k+1)}$ & $(\log (N T))^{-1 / k}$ & $N^{-2 /(2 k+1)}$ & $(\log N)^{-1 / k}$ \\
$r_{M}^{\star}$ & $(N T)^{-k /(2 k+1)}$ & $(N T)^{-1 / 2}(\log (N T))^{1 /(2 k)}$ & $N^{-2 k /(2 k+1)}$ & $N^{-1}(\log N)^{1 /(2 k)}$ \\
\hline \multicolumn{4}{c}{ TABLE 2} \\
\multicolumn{4}{c}{ A summary of $h^{\star}$ and $r_{M}^{\star}$ in $k$-order PDK and $k$-order EDK. }
\end{tabular}

\subsection{Asymptotic Normality}

In this section, we will derive the asymptotic normality for $\widehat{g}$ in the proposed RKHS framework which can be used to construct the confidence interval for $g(x)$ at any $x \in \mathcal{X}$. Our results are applicable in a general class of models including nonparametric models, semiparametric models or additive models. This is in sharp contrast to Su and Jin (2012) whose results were obtained in nonparametric sieve estimation. Our asymptotic normality result relies on the following theorem which characterizes the leading term of $\widehat{g}-g_{0}$. Define $D_{m}=\sum_{\nu=m+1}^{\infty} 1 /\left(1+\eta \rho_{\nu}\right)$ for any $m \geq 0$.

Theorem 4.2 (FBR for Homogeneous Model). Suppose that Assumptions A2, A4 and A5 hold, and $h^{-1}=o_{P}\left(N^{1 / 2}\right)$. Furthermore, there exists a sequence of positive integers $m=m_{M}$ such that $D_{m}=o_{P}(1)$. Then for any $x_{0} \in \mathcal{X}$, we have

$$
\sqrt{N T} A_{N T}\left|\widehat{g}_{0}\left(x_{0}\right)-g_{0}\left(x_{0}\right)+S_{M, \eta}\left(g_{0}\right)\left(x_{0}\right)-W_{\eta} g_{0}\left(x_{0}\right)\right|=O_{P}\left(\frac{b_{N, p}}{h}\left(\frac{1}{\sqrt{N} h}+\frac{\sqrt{T}}{N h}+\sqrt{\frac{T \eta}{h}}\right)\right) .
$$

Furthermore, if $b_{N, p}=o_{P}\left(N^{1 / 2} h^{2}\right), b_{N, p}=o_{P}\left(N h^{2} T^{-1 / 2}\right)$ and $b_{N, p}=o_{P}\left(h^{3 / 2}(T \eta)^{-1 / 2}\right)$, then

$$
\sqrt{N T} A_{N T}\left|\widehat{g}_{0}\left(x_{0}\right)-g_{0}\left(x_{0}\right)+S_{M, \eta}\left(g_{0}\right)\left(x_{0}\right)-W_{\eta} g_{0}\left(x_{0}\right)\right|=o_{P}(1) .
$$


It follows from Theorem 4.2 that $\widehat{g}\left(x_{0}\right)-g_{0}\left(x_{0}\right)$ and $S_{M, \eta}\left(g_{0}\right)\left(x_{0}\right)-W_{\eta} g_{0}\left(x_{0}\right)$ are asymptotically equivalent. The latter will be used to derive the limit distribution of $\widehat{g}\left(x_{0}\right)$, i.e., Theorem 4.3 below. Let $\gamma_{2}=\left(\gamma_{21}^{\prime}, \gamma_{22}^{\prime}, \ldots, \gamma_{2 N}^{\prime}\right)^{\prime}$, a $q_{2} N$-vector of unknown factor loadings.

Theorem 4.3. Suppose that Assumptions A2, A4 and A5 hold, and $h^{-1}=o_{P}\left(N^{1 / 2}\right)$. Furthermore, there exists a sequence of positive integers $m=m_{M}$ with $m=o\left(N^{1 / 2}\right)$ and $m T \lambda_{\max }\left(\gamma_{2} \gamma_{2}^{\prime}\right)=$ $o(N)$ such that $D_{m}=o_{P}\left(h^{1 / 2}\right)$ and $D_{m}^{2} T=o_{P}(N h)$. Then, for all $x_{0} \in \mathcal{X}$, it follows that

$$
\sqrt{N T} A_{N T}\left(\widehat{g}\left(x_{0}\right)-g_{0}\left(x_{0}\right)+W_{\eta} g_{0}\left(x_{0}\right)\right) \stackrel{d}{\rightarrow} N\left(0, \sigma_{\epsilon}^{2}\right), \text { as } M \rightarrow \infty
$$

where $A_{N T}=A_{N T}\left(x_{0}\right) \equiv\left(\frac{1}{N T} \sum_{i=1}^{N} K_{\mathbb{X}_{i}}^{\prime}\left(x_{0}\right) P K_{\mathbb{X}_{i}}\left(x_{0}\right)\right)^{-1 / 2}$.

Theorem 4.3 shows that $\widehat{g}\left(x_{0}\right)$ is asymptotically normal at any $x_{0} \in \mathcal{X}$. The rate conditions $h=o_{P}\left(N^{1 / 2}\right), m=o\left(N^{1 / 2}\right), D_{m}=o_{P}\left(h^{1 / 2}\right)$ and $D_{m}^{2} T=o_{P}(N h)$ are reasonable and can be verified in concrete settings. For instance, when $T \leq N$, for $k$-order PDK, the conditions hold if $h \asymp(N T)^{-1 /(2 k+1)}, m=N^{1 / 2} / \log (N)$, and correspondingly, $D_{m} \asymp h^{-2 k} m^{-2 k+1}$. For $k$-order EDK, the conditions hold if $h \asymp(\log (N T))^{-1 / k}, m=N^{1 / 4}$, and correspondingly, $D_{m} \asymp$ $e^{-c N^{k / 4}} T N^{(5-k) / 4}$. The condition $m T \lambda_{\max }\left(\gamma_{2} \gamma_{2}^{\prime}\right)=o(N)$ says that the signal of the unobserved factors is not strong so that the asymptotic normal part from $\widehat{g}$ can be filtered out.

Nonetheless, $\widehat{g}\left(x_{0}\right)$ does not converge to the truth due to the bias $W_{\eta} g_{0}\left(x_{0}\right)$. Following Shang and Cheng (2015), it can be verified that $W_{\eta} g_{0}\left(x_{0}\right)=o_{P}\left((\eta / h)^{1 / 2}\right)$. Therefore, we need to assume $N T \eta A_{N T}^{2}=O_{P}(h)$ for bias correction, a version of "undersmoothing condition."

Corollary 4.4. Suppose that the conditions in Theorem 4.3 are satisfied and $N T \eta A_{N T}^{2}=O_{P}(h)$. Then, as $M \rightarrow \infty, \sqrt{N T} A_{N T}\left(\widehat{g}\left(x_{0}\right)-g_{0}\left(x_{0}\right)\right) \stackrel{d}{\rightarrow} N\left(0, \sigma_{\epsilon}^{2}\right)$.

Corollary 4.4 provides asymptotic normality for $\widehat{g}\left(x_{0}\right)$ where the estimator converges to the truth. We can show that the undersmoothing condition $N T \eta A_{N T}^{2}=o_{P}(h)$ holds true when we properly choose $h$ with $h=o\left(h^{\star}\right)$ in both PDK and EDK. A direct consequence is the following $1-\alpha$ confidence interval for $g\left(x_{0}\right)$ :

$$
\widehat{g}\left(x_{0}\right) \pm z_{1-\alpha / 2} \frac{\sigma_{\varepsilon}}{\sqrt{N T} A_{N T}} .
$$

In practice, we estimate $\sigma_{\varepsilon}^{2}$ by $\widehat{\sigma}_{\varepsilon}^{2}=\frac{1}{N\left(T-q_{1}-d\right)} \sum_{i=1}^{N}\left(Y_{i}-\tau_{i} \widehat{g}\right)^{\prime} P\left(Y_{i}-\tau_{i} \widehat{g}\right)$, which is consistent as demonstrated in the following result. The rate conditions in Proposition 4.5 hold true when $h \asymp h^{\star}$ in both PDK and EDK.

Proposition 4.5. Suppose that Assumptions A2, A4, A5 hold. Moreover, $b_{N, p}=o_{P}\left(N^{1 / 2} h\right), h^{-1}=$ $o_{P}\left((N T)^{1 / 2}\right), h^{-1}=o_{P}(N)$ and $\eta=o_{P}(h)$. Then $\widehat{\sigma}_{\varepsilon}^{2} \rightarrow \sigma_{\epsilon}^{2}$ in probability, as $M \rightarrow \infty$.

Remark 4.2. It is of interest to apply our results to RKHS of delicate structures, e.g., additivity. Suppose that, for $l=1, \ldots, r, \mathcal{H}_{l}$ is an $R K H S$ with inner products and reproducing kernels denoted $\langle\cdot, \cdot\rangle_{\mathcal{H}_{l}}$ and $\bar{K}_{l}$, respectively. Define $\mathcal{H}=\left\{g_{1}\left(x_{1}\right)+\cdots+g_{r}\left(x_{r}\right): g_{1} \in \mathcal{H}_{1}, \cdots, g_{r} \in \mathcal{H}_{r}\right\}$. Then $\mathcal{H}$ 
is an Additive RKHS with kernel $\bar{K}\left(\left(x_{1}, \ldots, x_{r}\right),\left(y_{1}, \ldots, y_{r}\right)\right) \equiv \bar{K}_{1}\left(x_{1}, y_{1}\right)+\cdots+\bar{K}_{1}\left(x_{r}, y_{r}\right)$ and inner product $\langle f, g\rangle_{\mathcal{H}} \equiv\left\langle f_{1}, g_{1}\right\rangle_{\mathcal{H}_{1}}+\cdots+\left\langle f_{r}, g_{r}\right\rangle_{\mathcal{H}_{r}}$. In particular, $(\mathcal{H}, \bar{K})$ becomes Partial Linear if some of $\mathcal{H}_{l}$ 's are generated by linear kernels, i.e., 1-order polynomial.

Remark 4.3. When $g$ is partial linear, i.e., $g\left(x_{1}, x_{2}\right)=x_{1} \beta+g_{2}\left(x_{2}\right)$, (4.7) can be used to construct a confidence interval for $\beta$. To illustrate this, suppose $x_{1}, x_{2}$ are univariate for simplicity. Choose $x_{2}$ such that $g_{2}\left(x_{2}\right)=0$, then $\beta=g\left(1, x_{2}\right)$. By (4.7), the $1-\alpha$ confidence interval for $\beta$ is

$$
\widehat{g}\left(1, x_{2}\right) \pm z_{1-\alpha / 2} \frac{\widehat{\sigma}_{\varepsilon}}{\sqrt{N T} A_{N T}} .
$$

Extensions can be easily done when $x_{1}, x_{2}$ are multidimensional.

\section{Numerical Study}

We examine our methods using simulated datasets and a real dataset.

\subsection{Simulation}

A comparison will be first performed between our estimation procedure and Su and Jin's sieve estimation. We considered the same data generating process as Su and Jin (2012) for fair comparison. That is, the data were generated as follows: for $i \in[N], t \in[T]$,

$$
\begin{aligned}
y_{i t} & =g_{i}\left(x_{i t, 1}, x_{i t, 2}\right)+\gamma_{1 i}+\gamma_{2 i, 1} f_{2 t, 1}+\gamma_{2 i, 2} f_{2 t, 2}+\epsilon_{i t}, \\
g_{i}\left(x_{i t, 1}, x_{i t, 2}\right) & =\exp \left(x_{i t, 1}\right) /\left(1+\exp \left(x_{i t, 1}\right)\right)+\delta_{i}\left(0.5 x_{i t, 2}-0.25 x_{i t, 2}^{2}\right), \\
x_{i t, s} & =\Gamma_{1 i, s}+\Gamma_{2 i, s 1} f_{2 t, 1}+\Gamma_{2 i, s 2} f_{2 t, 2}+v_{i t, s}, s=1,2,
\end{aligned}
$$

where $\epsilon_{i t}=\rho_{i} \epsilon_{i, t-1}+\sigma_{i}\left(1-\rho_{i}^{2}\right) \xi_{i t}$ with $\rho_{i}, \sigma_{i}^{2} \stackrel{\text { iid }}{\sim} U n i f[0,0.95]$ and $\xi_{i t} \stackrel{i i d}{\sim} N(0,1) ; v_{i t, 1}, v_{i t, 2}$ were generated similar to $\epsilon_{i t} ; \delta_{i} \stackrel{i i d}{\sim} U n i f[0,1] ; f_{2 t, s}=0.5 f_{2, t-1, s}+\left(1-0.5^{2}\right)^{1 / 2} \zeta_{t, s}$ with $\zeta_{t, s} \stackrel{i i d}{\sim} N(0,1)$ for $s=1,2 ; \gamma_{1 i}=0.5 T^{-1} \sum_{t=1}^{T} x_{i t, 1}+0.5 T^{-1} \sum_{t=1}^{T} x_{i t, 2}$ and

$$
\gamma_{21}, \ldots, \gamma_{2 N}, \Gamma_{11}, \ldots, \Gamma_{1 N} \stackrel{i i d}{\sim} N\left(\left(\begin{array}{l}
0 \\
0
\end{array}\right),\left(\begin{array}{cc}
1 & 0.5 \\
0.5 & 1
\end{array}\right)\right)
$$

the entries of $\Gamma_{2 i}$ were generated from a multivariate normal distribution with mean $(1,0,0,1)$ and identity covariance matrix.

We chose an additive RKHS with kernel $\bar{K}\left(\left(x_{1}, x_{2}\right),\left(y_{1}, y_{2}\right)\right)=\bar{K}_{1}\left(x_{1}, y_{1}\right)+\bar{K}_{2}\left(x_{2}, y_{2}\right)$, where $\bar{K}_{1}$ is Gaussian kernel and $\bar{K}_{2}$ is a 2-order polynomial kernel. See Section 2.2 for definitions of these kernels. The smoothing parameter $\eta$ was chosen by the proposed GCV; see Remark 3.1. We considered $N=25,50,100$ in both heterogeneous and homogeneous cases, whereas $T=25,50,100$ in heterogeneous case and $T=8,25,100$ in homogeneous case. Mean squared errors (MSE) were computed based on 1000 replications. 
Table 3 compares our estimator $\widehat{g}$ with Su and Jin's sieve estimator $\widehat{g}_{\text {sieve }}$. We observe that, in heterogeneous setting, our estimator yields slightly smaller MSE when $N, T \leq 50$, and becomes comparable with $\widehat{g}_{\text {sieve }}$ when $N$ or $T$ is 100 . In homogeneous setting, it can be seen that our estimator yields slightly smaller MSE when $T=8$, and becomes comparable when $T=25,100$.

TABLE 3

MSE of two estimators in various settings.

\begin{tabular}{|c|c|c|c|c|c|c|c|c|}
\hline \multirow{3}{*}{$\begin{array}{l}\text { Estimator } \\
\widehat{g}\end{array}$} & \multicolumn{4}{|c|}{ Heterogeneous Setting } & \multicolumn{4}{|c|}{ Homogeneous Setting } \\
\hline & $\mathrm{N} / \mathrm{T}$ & 25 & 50 & 100 & $\mathrm{~N} / \mathrm{T}$ & 8 & 25 & 100 \\
\hline & 25 & 0.813 & 0.536 & 0.419 & 25 & 0.291 & 0.219 & 0.035 \\
\hline \multirow{5}{*}{$\widehat{g}_{\text {sieve }}$} & 50 & 0.793 & 0.518 & 0.394 & 50 & 0.171 & 0.133 & 0.030 \\
\hline & 100 & 0.980 & 0.545 & 0.402 & 100 & 0.123 & 0.118 & 0.019 \\
\hline & 25 & 1.061 & 0.736 & 0.538 & 25 & 0.528 & 0.245 & 0.143 \\
\hline & 50 & 0.932 & 0.646 & 0.457 & 50 & 0.344 & 0.164 & 0.095 \\
\hline & 100 & 0.996 & 0.674 & 0.47 & 100 & 0.245 & 0.115 & 0.065 \\
\hline
\end{tabular}

Next, we examined the proposed confidence interval (4.7). We only considered the homogeneous setting (4.2) with $N=25,50,100, T=8,25$, and "true" function $g(x)=0.6 \beta_{30,17}(x)+0.4 \beta_{3,11}(x)$, where $\beta_{a, b}(\cdot)$ is the beta density function with shape and scale $a$ and $b ; \epsilon_{i t}, v_{i t} \stackrel{\text { iid }}{\sim} N(0,1) ; f_{1 t, s}=$ $0.5 f_{1 t-1, s}+\left(1-0.5^{2}\right)^{1 / 2} \zeta_{t, s}$ for $s=1,2$, where $\zeta_{t, 1}, \zeta_{t, 2} \stackrel{i i d}{\sim} N(0,1) ; f_{2 t, s}$ was generated the same way as $f_{1 t, s} ; \gamma_{1 i}=T^{-1} \sum_{t=1}^{T} x_{i t}$ and $\Gamma_{1 i} \stackrel{i i d}{\sim} N(0,1) ; \gamma_{2 i} \stackrel{i i d}{\sim} N(0,1)$ and $\Gamma_{2 i} \stackrel{i i d}{\sim} N(1,1)$.

Confidence intervals for $g(x)$ at $x \in[0,1]$ were constructed based on Gaussian kernel and a 10-order polynomial kernel. The smoothing parameter was selected by the proposed GCV; see Remark 4.1. The coverage probabilities (CP) of the intervals were examined based on 1000 independent replications. Figures 1 and 2 display the $\mathrm{CP}$ of the $95 \%$ confidence intervals for $g(x)$ at 100 evenly spaced points in [0,1] based on Gaussian kernel and polynomial kernel, respectively. It can be seen that, when $N=100$ or $N=50, T=25$, the $\mathrm{CP}$ approaches the $95 \%$ nominal level at any $x \in[0,1]$, demonstrating the validity of the confidence intervals. When $N=25$ or $N=50, T=8$, the $\mathrm{CP}$ is significantly less than the nominal level at $x \in[0,0.2]$. This is due to the peaks/trouts of the true function which affect the small sample performance of the intervals. Such effect quickly vanishes in large sample setting, e.g., $N=100$ or $N=50, T=25$.

\subsection{Export Productivity Premium}

In this section, we apply our method to examine the firm-level productivity difference between exporters and non-exporters based on a real dataset from computer and peripheral equipment manufacturing industry of Chinese Industrial Enterprises Database. The data include observations collected from $N=100$ continuously operating firms in $T=9$ years (1998 to 2007). For firm $i$ in year $t, Y_{i t}$ is the log gross output, $X_{i t}=\left(X_{i t 1}, X_{i t 2}, X_{i t 3}, X_{i t 4}\right)$ with $X_{i t 1}$ the log capital defined as the net fixed asset, $X_{i t 2}$ the $\log$ materials defined as the value of the intermediate inputs, $X_{i t 3}$ the log labor defined as the total wage bill plus benefits, and $X_{i t 4}$ the export intensity defined as 

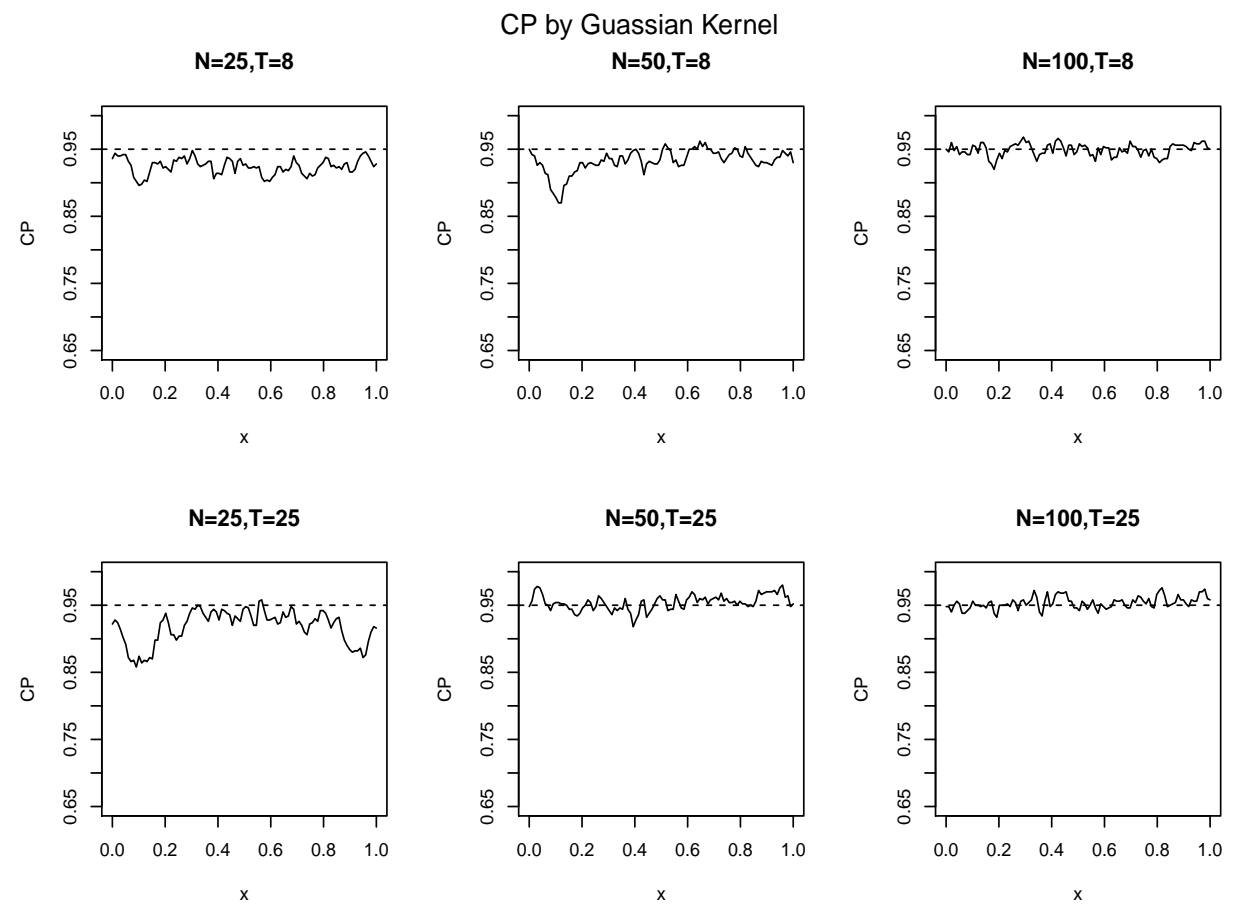

FIG 1. CP of the 95\% confidence intervals for $g(x)$ at $x \in[0,1]$ based on Gaussian kernel. Dashed lines indicate $95 \%$ nominal level.

the ratio of the export value to the gross output value. The aim is to investigate a relationship between $X_{i t}$ and $Y_{i t}$.

To enhance model flexibility, suppose that the log gross output and the export intensity are nonlinearly related. This leads us to consider the following model

$$
Y_{i t}=\beta_{1} X_{i t 1}+\beta_{2} X_{i t 2}+\beta_{3} X_{i t 3}+f\left(X_{i t 4}\right)+\gamma_{1 i}+\gamma_{2 i} f_{2 t}+\text { error }
$$

where $\beta_{1}, \beta_{2}, \beta_{3}$ are unknown regression coefficients and $f$ is unknown belonging to an RKHS $\mathcal{H}$ which represents productivity difference between exporters and non-exporters. The variables $f_{2 t}$ represent the unobserved common shocks, such as unobserved policy changes, and $\gamma_{1 i}, \gamma_{2 i}$ represent the individual specific responses to factor $f_{1 t}=1$ and $f_{2 t}$. The semiparametric structure of the regression function $g\left(X_{i t}\right) \equiv X_{i t 1} \beta_{1}+\beta_{2} X_{i t 2}+\beta_{3} X_{i t 3}+f\left(X_{i t 4}\right)$ can be naturally incorporated in an additive RKHS generated by a polynomial kernel and a general RKHS $\mathcal{H}$; see Remark 4.2. In practice, we chose $\mathcal{H}$ as generated by linear kernel or polynomial kernel.

Table 4 summarizes the estimates and $95 \%$ confidence intervals of $\beta_{1}, \beta_{2}, \beta_{3}$. The intervals were calculated based on (4.8). Overall, the results based on linear kernel and polynomial kernel are quite similar. The confidence intervals all exclude zero indicating the significance of the linear predictors, consistent with literature about Chinese manufacturing industries (e.g. Hashiguchi, 


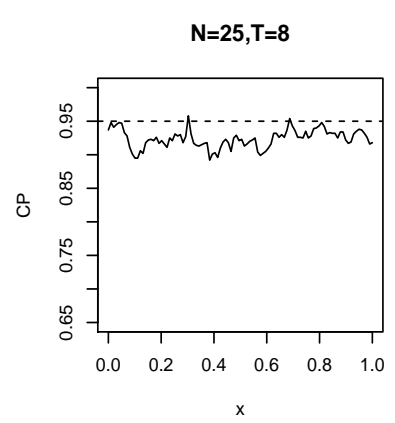

\section{CP by Polynomial Kernel}

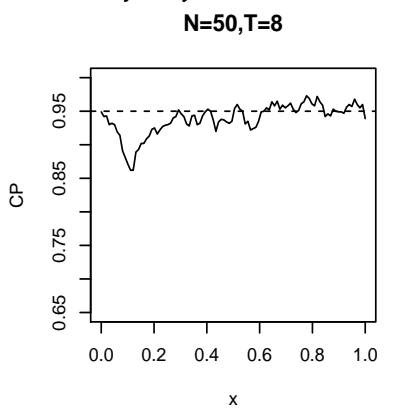

$\mathrm{N}=25, \mathrm{~T}=25$

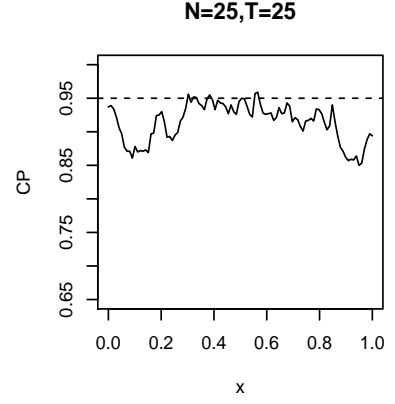

$\mathrm{N}=50, \mathrm{~T}=25$

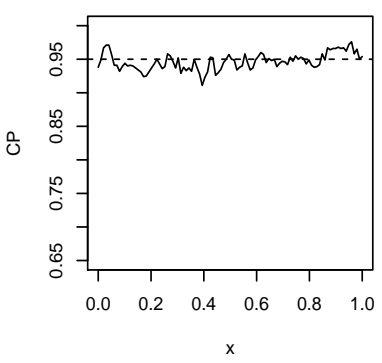

$\mathrm{N}=100, \mathrm{~T}=8$

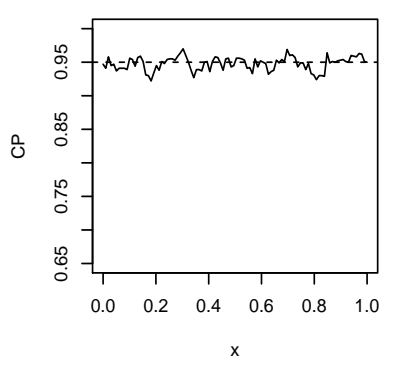

$\mathrm{N}=\mathbf{1 0 0 , T}=\mathbf{2 5}$

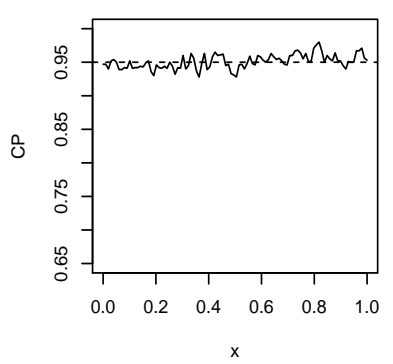

FIG 2. CP of the $95 \%$ confidence intervals for $g(x)$ at $x \in[0,1]$ based on polynomial kernel. Dashed lines indicate $95 \%$ nominal level.

TABLE 4

Estimation and $95 \%$ confidence intervals for $\beta_{1}, \beta_{2}, \beta_{3}$ based on two kernels.

\begin{tabular}{llllll}
\hline \hline \multicolumn{3}{c}{ Linear Kernel } & \multicolumn{3}{c}{ Polynomial Kernel } \\
& Estimate & $95 \%$ CI & & Estimate & $95 \%$ CI \\
$\beta_{1}$ & 0.1022 & {$[0.0624,0.1420]$} & $\beta_{1}$ & 0.1010 & {$[0.0606,0.1414]$} \\
$\beta_{2}$ & 0.0994 & {$[0.0672,0.1316]$} & $\beta_{2}$ & 0.0989 & {$[0.0663,0.1315]$} \\
$\beta_{3}$ & 0.7300 & {$[0.6958,0.7642]$} & $\beta_{3}$ & 0.7395 & {$[0.7049,0.7741]$} \\
\hline \hline
\end{tabular}

2015). Figure 3 displays the $95 \%$ confidence intervals for export productivity premium versus export intensity, based on linear kernel (left panel) and polynomial kernel (right panel). The red dashed lines display the upper and lower bounds of the intervals, and the central dark lines demonstrate the estimations of $f$. Overall, the estimations of $f$ are both increasing, consistent with the folklore that "exports stimulate productivity," e.g., Melitz (2003). The red dashed lines are above zero, indicating the significance of the export intensity effect on productivity.

\section{APPENDIX}

This appendix contains the proofs of the main results. In Section A.1, a proof of convergence rate in heterogeneous model is provided (Theorem 3.1) and some auxiliary lemmas are stated. In Section A.2, we prove FBR for heterogeneous model (Theorem 3.2) and joint asymptotic distributions 

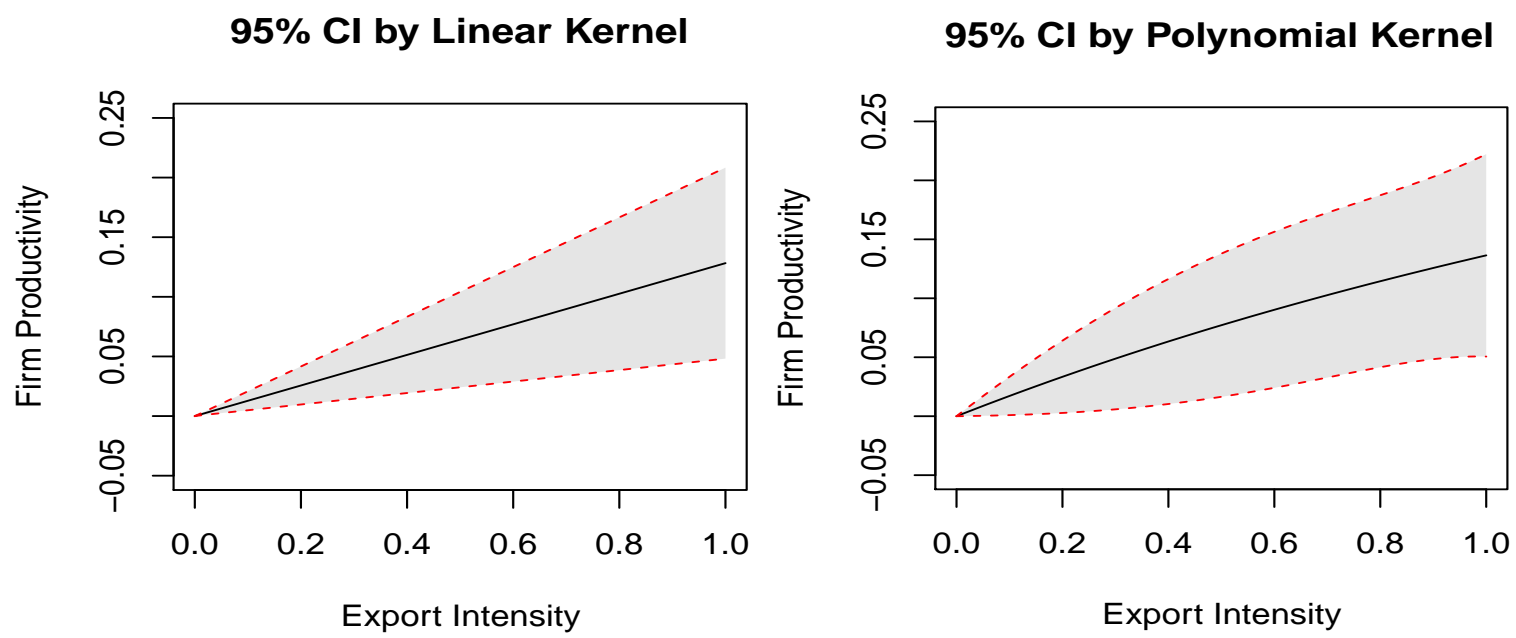

FIG 3. 95\% confidence intervals for firm productivity versus export intensity.

of our estimators (Theorems 3.3 and 3.4). Section A.3 includes the proof of convergence rate in homogeneous model (Theorem 4.1) as well as some auxiliary lemmas. In Section A.4, proofs of FBR in homogeneous model (Theorem 4.2) and corresponding asymptotic normality (Theorem 4.3) are given. We also show that the variance estimator is consistent.

\section{A.1. Proofs in Section 3.2}

In this section, we derive the rate of convergence for our estimator in the heterogeneous setting, i.e., Theorem 3.1. Before proving the results, we provide some preliminary results.

Lemma A.1. For any $\theta \in \Theta_{i}, D S_{i, M, \eta_{i}}^{\star}(\theta)=i d$, where $S_{i, M, \eta_{i}}^{\star}(\theta)=E\left\{S_{i, M, \eta_{i}}(\theta)\right\}$.

Lemma A.2. There exist universal constants $C_{1}, C_{2}, \ldots, C_{N}$ such that,

$$
\begin{aligned}
\left\|R_{i} U_{i t}\right\|_{i}^{2} & \leq C_{i}^{2}\left(h_{i}^{-1}+Z_{t}^{\prime} Z_{t}\right), \quad \text { for any } i \in[N], t \in[N], \\
\|\theta\|_{i, \text { sup }} & \leq C_{i}\left(1+h_{i}^{-1 / 2}\right)\|\theta\|_{i}, \quad \text { for any } \theta \in \Theta_{i} .
\end{aligned}
$$

Proposition A.1. Under Assumption A2, as $T \rightarrow \infty, \max _{1 \leq t \leq T}\left\|Z_{t}\right\|_{2}=O_{P}\left(T^{1 / \alpha}\right)$.

The following proposition holds for both (1) $T, N \rightarrow \infty$; (2) $N \rightarrow \infty, T$ is fixed. That is, the result holds for $M \rightarrow \infty$.

Proposition A.2. Let Assumptions A2-A3 hold. For $i \in[N]$ and $t \in[T]$, let $p_{i}=p_{i}(M) \geq 1$ be a deterministic sequence indexed by $M$, and let $\psi_{i, M, t}\left(U_{i t} ; \theta\right)$ be a real-valued function defined on $\Theta_{i}$ such that $\psi_{i, M, t}\left(U_{i t} ; 0\right) \equiv 0$, and for any $\theta_{1}, \theta_{2} \in \Theta_{i}$,

$$
\left\|\left(\psi_{i, M, t}\left(U_{i t} ; \theta_{1}\right)-\psi_{i, M, t}\left(U_{i t} ; \theta_{2}\right)\right) R_{i} U_{i t}\right\|_{i} \leq\left\|\theta_{1}-\theta_{2}\right\|_{i, \text { sup }}
$$


Then there exists a universal constant $C_{0}>0$ such that, as $N \rightarrow \infty$,

$$
P\left(\max _{i \in[N]} \sup _{\theta \in \mathcal{G}_{i}\left(p_{i}\right)} \frac{\sqrt{T}\left\|\mathbb{Z}_{i M}(\theta)\right\|_{i}}{\sqrt{T} J_{i}\left(p_{i},\|\theta\|_{i, \text { sup }}\right)+1} \geq C_{0} \sqrt{\log N+\log \log \left(T J_{i}\left(p_{i}, 1\right)\right)}\right) \rightarrow 0
$$

where

$$
\mathbb{Z}_{i M}(\theta)=\frac{1}{\sqrt{T}} \sum_{t=1}^{T}\left[\psi_{i, M, t}\left(U_{i t} ; \theta\right) R_{i} U_{i t}-E\left(\psi_{i, M, t}\left(U_{i t} ; \theta\right) R_{i} U_{i t}\right)\right], \theta \in \Theta_{i} .
$$

Proofs of Lemmas A.1 and A.2, Propositions A.1 and A.2 can be found in supplement document.

Proof of Theorem 3.1. Since $Y_{i t}=g_{i 0}\left(X_{i t}\right)+Z_{t}^{\prime} \beta_{i 0}+e_{i t}$, it follows that

$$
S_{i, M, \eta_{i}}^{\star}\left(\theta_{i 0}\right)=E\left\{S_{i, M, \eta_{i}}\left(\theta_{i 0}\right)\right\}=E\left\{-\frac{1}{T} \sum_{t=1}^{T} e_{i t} R_{i} U_{i t}+P_{i} \theta_{i 0}\right\}
$$

Also $e_{i t}=\epsilon_{i t}-\gamma_{2 i}^{\prime}\left(\bar{\Gamma}_{2} \bar{\Gamma}_{2}^{\prime}\right)^{-1} \bar{\Gamma}_{2} \bar{v}_{t}=\epsilon_{i t}-\Delta_{i} \bar{v}_{t}$, so we have

$$
\begin{aligned}
\left\|S_{i, M, \eta_{i}}^{\star}\left(\theta_{i 0}\right)\right\|_{i} & =\left\|E\left\{\left(\epsilon_{i t}-\Delta_{i} \bar{v}_{t}\right) R_{i} U_{i t}-P_{i} \theta_{i 0}\right\}\right\|_{i} \\
& \leq \| E\left\{\left(\epsilon_{i t}-\Delta_{i} \bar{v}_{t}\right) R_{i} U_{i t}\left\|_{i}+\right\| P_{i} \theta_{i 0} \|_{i}\right. \\
& =\sup _{\|\theta\|_{i}=1}\left|\left\langle E\left\{\left(\epsilon_{i t}-\Delta_{i} \bar{v}_{t}\right) R_{i} U_{i t}\right\}, \theta\right\rangle_{i}\right|+\left\|P_{i} \theta_{i 0}\right\|_{i} \\
& =\sup _{\|\theta\|_{i}=1}\left|E\left\{\left(\epsilon_{i t}-\Delta_{i} \bar{v}_{t}\right)\left(g\left(X_{i t}\right)+Z_{t}^{\prime} \beta_{i}\right)\right\}\right|+\left\|P_{i} \theta_{i 0}\right\|_{i} \\
& =\sup _{\|\theta\|_{i}=1}\left|E\left\{\Delta_{i} \bar{v}_{t}\left(g\left(X_{i t}\right)+Z_{t}^{\prime} \beta_{i}\right)\right\}\right|+\left\|P_{i} \theta_{i 0}\right\|_{i} .
\end{aligned}
$$

Since

$$
\left|g\left(X_{i t}\right)+Z_{t}^{\prime} \beta_{i}\right| \leq\left(1+\left\|Z_{t}\right\|_{2}\right)\|\theta\|_{i, \text { sup }} \leq C_{i}\left(1+\left\|Z_{t}\right\|_{2}\right)\left(1+h_{i}^{-1 / 2}\right)\|\theta\|_{i}
$$

and

$$
E\left\{\Delta_{i} \bar{v}_{t} h_{i}^{-1 / 2}\right\} \leq E\left\{\left(\Delta_{i} \bar{v}_{t}\right)^{2}\right\}^{1 / 2} h_{i}^{-1 / 2}=O\left(\left(N h_{i}\right)^{-1 / 2}\right),
$$

there exists a constant $C^{\prime}$, such that

$$
\sup _{\|\theta\|_{i}=1}\left|E\left\{\Delta_{i} \bar{v}_{t}\left(g\left(X_{i t}\right)+Z_{t}^{\prime} \beta_{i}\right)\right\}\right| \leq \frac{C^{\prime}}{\left(N h_{i}\right)^{1 / 2}}
$$

In the meantime, we have

$$
\left\|P_{i} \theta_{i 0}\right\|_{i}=\sup _{\|\theta\|_{i}=1}\left|\left\langle P_{i} \theta_{i 0}, \theta\right\rangle_{i}\right|=\sup _{\|\theta\|_{i}=1}\left|\eta_{i}\left\langle g_{i 0}, g\right\rangle_{i}\right| \leq \sqrt{\eta_{i}}\left\|g_{i 0}\right\|_{\mathcal{H}_{i}}, \quad i \in[N] .
$$

Consider an operator

$$
T_{1 i}(\theta)=\theta-S_{i, M, \eta_{i}}^{\star}\left(\theta+\theta_{i 0}\right), \quad \theta \in \Theta_{i} .
$$

By Lemma A.1 we have for any $\theta \in \Theta_{i}$,

$$
T_{1 i}(\theta)=\theta-D S_{i, M, \eta_{i}}^{\star}\left(\theta_{i 0}\right) \theta-S_{i, M, \eta_{i}}^{\star}\left(\theta_{i 0}\right)=-S_{i, M, \eta_{i}}^{\star}\left(\theta_{i 0}\right) .
$$


Since $T_{1 i}$ takes constant value and by (A.3) and (A.4), $T_{1 i}$ is a contraction mapping from $\mathbb{B}_{i}\left(\sqrt{\eta_{i}}\left\|g_{i 0}\right\|_{\mathcal{H}_{i}}+\frac{C^{\prime}}{\left(N h_{i}\right)^{1 / 2}}\right)$ to itself, where $\mathbb{B}_{i}(r)$ represents the $r$-ball in $\left(\Theta_{i},\|\cdot\|_{i}\right)$. By Contraction mapping theorem, there exists a unique fixed point $\theta^{\prime} \in \mathbb{B}_{i}\left(\sqrt{\eta_{i}}\left\|g_{i 0}\right\|_{\mathcal{H}_{i}}+\frac{C^{\prime}}{\left(N h_{i}\right)^{1 / 2}}\right)$ such that $T_{1 i}\left(\theta^{\prime}\right)=\theta^{\prime}$. Let $\theta_{\eta_{i}}=\theta^{\prime}+\theta_{i 0}$, then $S_{i, M, \eta_{i}}^{\star}\left(\theta_{\eta_{i}}\right)=0$. Obviously, $\left\|\theta_{\eta_{i}}-\theta_{i 0}\right\|_{i} \leq \sqrt{\eta_{i}}\left\|g_{i 0}\right\|_{\mathcal{H}_{i}}+\frac{C^{\prime}}{\left(N h_{i}\right)^{1 / 2}}$.

We fix an $i \in[N]$ and assume both $T, N$ to approach infinity. Let $\mathcal{E}_{M}=\left\{\max _{1 \leq t \leq T}\left\|Z_{t}\right\|_{2} \leq\right.$ $\left.\widetilde{C} T^{1 / \alpha}\right\}$. Proposition A.1 says that when $\widetilde{C}$ is large, $\mathcal{E}_{M}$ has probability approaching one. Write $\mathcal{E}_{M, t}=\left\{\left\|Z_{t}\right\|_{2} \leq \widetilde{C} T^{1 / \alpha}\right\}$. Then $\mathcal{E}_{M}=\cap_{t=1}^{T} \mathcal{E}_{M, t}$. By Lemma A.2, $\mathcal{E}_{M, t}$ implies that $\left\|R_{i} U_{i t}\right\|_{i} \leq$ $C_{i}\left(h_{i}^{-1 / 2}+\widetilde{C} T^{1 / \alpha}\right)$.

Consider another operator

$$
T_{2 i}(\theta)=\theta-S_{i, M, \eta_{i}}\left(\theta_{\eta_{i}}+\theta\right), \quad \theta \in \Theta_{i} .
$$

For $i \in[N], t \in[T]$, define

$$
\psi_{i, M, t}\left(U_{i t} ; \theta\right)=\frac{\left\langle R_{i} U_{i t}, \theta\right\rangle_{i} I_{\mathcal{E}_{M, t}}}{\widetilde{C} C_{i} T^{1 / \alpha}\left(h_{i}^{-1 / 2}+\widetilde{C} T^{1 / \alpha}\right)}, \theta \in \Theta_{i} .
$$

It is easy to see that on $\mathcal{E}_{M}$, for any $\theta_{1}=\left(\beta_{1}, g_{1}\right), \theta_{2}=\left(\beta_{2}, g_{2}\right) \in \Theta_{i}$, by Proposition 2.1,

$$
\begin{aligned}
& \left\|\left(\psi_{i, M, t}\left(U_{i t} ; \theta_{1}\right)-\psi_{i M t}\left(U_{i t} ; \theta_{2}\right)\right) R_{i} U_{i t}\right\|_{i} \\
= & \frac{\left|\left\langle R_{i} U_{i t}, \theta_{1}-\theta_{2}\right\rangle_{i}\right| \times\left\|R_{i} U_{i t}\right\|_{i}}{C_{i} \widetilde{C} T^{1 / \alpha}\left(h_{i}^{-1 / 2}+\widetilde{C} T^{1 / \alpha}\right)} I_{\mathcal{E}_{M, t}} \\
= & \frac{\left|\left(g_{1}-g_{2}\right)\left(X_{i t}\right)+Z_{t}^{\prime}\left(\beta_{1}-\beta_{2}\right)\right| \times\left\|R_{i} U_{i t}\right\|_{i}}{C_{i} \widetilde{C} T^{1 / \alpha}\left(h_{i}^{-1 / 2}+\widetilde{C} T^{1 / \alpha}\right)} I_{\mathcal{E}_{M, t}} \\
\leq & \frac{\left\|\theta_{1}-\theta_{2}\right\|_{i, \text { sup }} \widetilde{C} T^{1 / \alpha} C_{i}\left(h_{i}^{-1 / 2}+\widetilde{C} T^{1 / \alpha}\right)}{C_{i} \widetilde{C} T^{1 / \alpha}\left(h_{i}^{-1 / 2}+\widetilde{C} T^{1 / \alpha}\right)} I_{\mathcal{E}_{M, t}} \leq\left\|\theta_{1}-\theta_{2}\right\|_{i, \text { sup }} .
\end{aligned}
$$

Notice the following decomposition:

$$
\begin{aligned}
T_{2 i}(\theta) & =\theta-S_{i, M, \eta_{i}}\left(\theta+\theta_{\eta_{i}}\right)+S_{i, M, \eta_{i}}\left(\theta_{\eta_{i}}\right)-S_{i, M, \eta_{i}}\left(\theta_{\eta_{i}}\right) \\
& =\theta-D S_{i, M, \eta_{i}}\left(\theta_{\eta_{i}}\right) \theta-S_{i, M, \eta_{i}}\left(\theta_{\eta_{i}}\right) .
\end{aligned}
$$

We first examine $S_{i, M, \eta_{i}}\left(\theta_{\eta_{i}}\right)$ as follows:

$$
\begin{aligned}
S_{i, M, \eta_{i}}\left(\theta_{\eta_{i}}\right)= & S_{i, M, \eta_{i}}\left(\theta_{\eta_{i}}\right)-E\left(S_{i, M, \eta_{i}}\left(\theta_{\eta_{i}}\right)\right) \\
= & -\frac{1}{T} \sum_{t=1}^{T}\left[\left(Y_{i t}-\left\langle R_{i} U_{i t}, \theta_{\eta_{i}}\right\rangle_{i}\right) R_{i} U_{i t}-E\left(\left(Y_{i t}-\left\langle R_{i} U_{i t}, \theta_{\eta_{i}}\right\rangle_{i}\right) R_{i} U_{i t}\right)\right] \\
= & -\frac{1}{T} \sum_{t=1}^{T}\left[e_{i t} R_{i} U_{i t}-E\left(e_{i t} R_{i} U_{i t}\right)\right] \\
& +\frac{1}{T} \sum_{t=1}^{T}\left[\left\langle R_{i} U_{i t}, \theta_{\eta_{i}}-\theta_{i 0}\right\rangle_{i} R_{i} U_{i t}-E\left(\left\langle R_{i} U_{i t}, \theta_{\eta_{i}}-\theta_{i 0}\right\rangle_{i} R_{i} U_{i t}\right)\right]
\end{aligned}
$$


Define $\xi_{i t}=e_{i t} R_{i} U_{i t}$. Following Dehling (1983, eqn. (3.2)) and Bradley (2005, eqn. (1.11)),

$$
\begin{aligned}
& E\left\|\sum_{t=1}^{T}\left[e_{i t} R_{i} U_{i t}-E\left(e_{i t} R_{i} U_{i t}\right)\right]\right\|_{i}^{2} \\
= & E\left\|\sum_{t=1}^{T}\left[\xi_{i t}-E\left(\xi_{i t}\right)\right]\right\|_{i}^{2} \\
= & \sum_{t, t^{\prime}=1}^{T}\left[E\left(\left\langle\xi_{i t}, \xi_{i t^{\prime}}\right\rangle_{i}\right)-\left\langle E\left(\xi_{i t}\right), E\left(\xi_{i t^{\prime}}\right)\right\rangle_{i}\right] \\
\leq & \sum_{t, t^{\prime}=1}^{T} 15\left(\phi\left(\left|t-t^{\prime}\right|\right) / 2\right)^{1-4 / \alpha} E\left(\left\|\xi_{i t}\right\|_{i}^{\alpha / 2}\right)^{4 / \alpha} .
\end{aligned}
$$

It follows from Assumption A2 (a), (c), (d), and Lemma A.2 that

$$
\begin{aligned}
E\left(\left\|\xi_{i t}\right\|_{i}^{\alpha / 2}\right)^{2} & =E\left(\left|e_{i t}\right|^{\alpha / 2}\left\|R_{i} U_{i t}\right\|_{i}^{\alpha / 2}\right)^{2} \\
& \leq E\left(\left|e_{i t}\right|^{\alpha / 2}\right) E\left(\left\|R_{i} U_{i t}\right\|_{i}^{\alpha}\right) \leq c_{0} h_{i}^{-\alpha / 2}
\end{aligned}
$$

where $c_{0}$ is an absolute constant. The existence of such $c_{0}$ is due to the fact $E\left(\left|e_{i t}\right|^{\alpha}\right)<\infty$ and $E\left(\left\|Z_{t}\right\|_{2}^{\alpha}\right)<\infty$. Therefore, it follows from Assumption A2 (b) that there exists an absolute constant $c_{1}$ such that

$$
E\left\|\sum_{t=1}^{T}\left[e_{i t} R_{i} U_{i t}-E\left(e_{i t} R_{i} U_{i t}\right)\right]\right\|_{i}^{2} \leq c_{1} T h_{i}^{-1} .
$$

Similarly, it can be shown that

$$
\begin{aligned}
& E\left\|\sum_{t=1}^{T}\left[\left\langle R_{i} U_{i t}, \theta_{\eta_{i}}-\theta_{i 0}\right\rangle_{i} R_{i} U_{i t}-E\left(\left\langle R_{i} U_{i t}, \theta_{\eta_{i}}-\theta_{i 0}\right\rangle_{i} R_{i} U_{i t}\right)\right]\right\|_{i}^{2} \\
\leq & \sum_{t, t^{\prime}=1}^{T} 15\left(\phi\left(\left|t-t^{\prime}\right|\right) / 2\right)^{1-4 / \alpha} E\left(\left\|R_{i} U_{i t}\right\|_{i}^{\alpha}\right)^{4 / \alpha}\left\|\theta_{\eta_{i}}-\theta_{i 0}\right\|_{i}^{2} \\
\leq & c_{1}^{\prime} T h_{i}^{-1},
\end{aligned}
$$

where $c_{1}^{\prime}$ is an absolute constant. The last step follows from Proposition A.2, i.e.,

$$
E\left(\left\|R_{i} U_{i t}\right\|_{i}^{\alpha}\right)=O\left(h_{i}^{-\alpha / 2}\right)
$$

and the fact $\left\|\theta_{\eta_{i}}-\theta_{i 0}\right\|_{i}^{2}=O\left(\eta_{i}+\frac{1}{N h_{i}}\right)$, and the condition $\eta_{i}+\frac{1}{N h_{i}}=O\left(h_{i}\right)$.

Therefore, we can choose $c_{2}$ to be large such that, with probability approaching one,

$$
\left\|S_{i, M, \eta_{i}}\left(\theta_{\eta_{i}}\right)\right\|_{i} \leq c_{2}\left(T h_{i}\right)^{-1 / 2}
$$

On $\mathcal{E}_{M, t}$, for any unequal $\theta_{1}, \theta_{2} \in \Theta_{i}$, define

$$
\theta=\frac{\theta_{1}-\theta_{2}}{C_{i}\left(1+h_{i}^{-1 / 2}\right)\left\|\theta_{1}-\theta_{2}\right\|_{i}} .
$$


Write $\theta=(\beta, g)$. Hence, by Lemma A.2 (A.2),

$$
\begin{aligned}
\|\theta\|_{i, \text { sup }} & \leq 1 \\
\eta_{i}\|g\|_{\mathcal{H}_{i}}^{2} & \leq\|\theta\|_{i}^{2}=\frac{\left\|\theta_{1}-\theta_{2}\right\|_{i}^{2}}{C_{i}^{2}\left(1+h_{i}^{-1 / 2}\right)^{2}\left\|\theta_{1}-\theta_{2}\right\|_{i}^{2}} \leq C_{i}^{-2} h_{i} .
\end{aligned}
$$

This means that $\theta \in \mathcal{G}_{i}\left(p_{i}\right)$ with $p_{i}=C_{i}^{-1}\left(\eta_{i}^{-1} h_{i}\right)^{1 / 2}$. Since $\eta_{i}^{-1} h_{i}$ tends to infinity as $(N, T)$ does, it is not of loss of generality to assume that $p_{i} \geq 1$. Define

$$
\mathbb{Z}_{i M}(\theta)=\frac{1}{\sqrt{T}} \sum_{t=1}^{T}\left[\psi_{i, M, t}\left(U_{i t} ; \theta\right) R_{i} U_{i t}-E\left(\psi_{i, M, t}\left(U_{i t} ; \theta\right) R_{i} U_{i t}\right)\right] .
$$

It follows from (A.5) and Proposition A.2 that, with probability approaching one,

$$
\sup _{\theta \in \mathcal{G}_{i}\left(p_{i}\right)} \frac{\sqrt{T}\left\|Z_{i M}(\theta)\right\|_{i}}{\sqrt{T} J_{i}\left(p_{i},\|\theta\|_{i, \text { sup }}\right)+1} \leq C_{0} \sqrt{\log N+\log \log \left(T J_{i}\left(p_{i}, 1\right)\right)} .
$$

Since $h_{i}=o(1)$, assume that $h_{i}^{-1} \geq 1$. It follows from Lemma A.2 (A.1) that

$$
\begin{aligned}
& \left\|E\left(\left\langle R_{i} U_{i t}, \theta\right\rangle_{i} I_{\mathcal{E}_{M, t}^{c}} R_{i} U_{i t}\right)\right\|_{i} \\
\leq & E\left(\left|\left\langle R_{i} U_{i t}, \theta\right\rangle_{i}\right| I_{\mathcal{E}_{M, t}^{c}}\left\|R_{i} U_{i t}\right\|_{i}\right) \\
\leq & E\left(\left(1+\left\|Z_{t}\right\|_{2}\right) I_{\mathcal{E}_{M, t}^{c}} C_{i}\left(h_{i}^{-1 / 2}+\left\|Z_{t}\right\|_{2}\right)\right) \\
\leq & C_{i} h_{i}^{-1 / 2} E\left(\left(1+\left\|Z_{t}\right\|_{2}\right)^{2} I_{\mathcal{E}_{M, t}^{c}}\right) \\
\leq & C_{i} h_{i}^{-1 / 2} E\left(\left(1+\left\|Z_{t}\right\|_{2}\right)^{\alpha}\right)^{2 / \alpha} P\left(\mathcal{E}_{M, t}^{c}\right)^{1-2 / \alpha} \\
\leq & C_{i} h_{i}^{-1 / 2} E\left(\left(1+\left\|Z_{t}\right\|_{2}\right)^{\alpha}\right)^{2 / \alpha}\left(\frac{1}{\widetilde{C}^{\alpha} T} E\left(\left\|Z_{t}\right\|_{2}^{\alpha}\right)\right)^{1-2 / \alpha} .
\end{aligned}
$$

Consequently, with probability approaching one, for any unequal $\theta_{1}, \theta_{2} \in \Theta_{i}$ On $\mathcal{E}_{M, t}$, it follows 
from (A.6) and (A.7) that

$$
\begin{aligned}
& \left\|T_{2 i}\left(\theta_{1}\right)-T_{2 i}\left(\theta_{2}\right)\right\|_{i} \\
= & \left\|-\frac{1}{T} \sum_{t=1}^{T}\left[\left\langle R_{i} U_{i t}, \theta_{1}-\theta_{2}\right\rangle_{i} R_{i} U_{i t}-E\left(\left\langle R_{i} U_{i t}, \theta_{1}-\theta_{2}\right\rangle_{i} R_{i} U_{i t}\right)\right]\right\|_{i} \\
= & \left\|-\frac{1}{T} \sum_{t=1}^{T}\left[\left\langle R_{i} U_{i t}, \theta\right\rangle_{i} R_{i} U_{i t}-E\left(\left\langle R_{i} U_{i t}, \theta\right\rangle_{i} R_{i} U_{i t}\right)\right] \times\right\| \theta_{1}-\theta_{2}\left\|_{i} C_{i}\left(1+h_{i}^{-1 / 2}\right)\right\|_{i} \\
= & \left\|\theta_{1}-\theta_{2}\right\|_{i} C_{i}\left(1+h_{i}^{-1 / 2}\right) \|\left(-\frac{1}{T} \sum_{t=1}^{T}\left[\left\langle R_{i} U_{i t}, \theta\right\rangle_{i} I_{\mathcal{E}_{M, t}} R_{i} U_{i t}-E\left(\left\langle R_{i} U_{i t}, \theta\right\rangle_{i} I_{\mathcal{E}_{M, t}} R_{i} U_{i t}\right)\right]\right. \\
& \left.+E\left(\left\langle R_{i} U_{i t}, \theta\right\rangle_{i} I_{\mathcal{E}_{M, t}^{c}} R_{i} U_{i t}\right)\right)\|\|_{i} \\
= & \left\|\theta_{1}-\theta_{2}\right\|_{i} C_{i}\left(1+h_{i}^{-1 / 2}\right)\left\|\left(-T^{-1 / 2} C_{i} \widetilde{C} T^{1 / \alpha}\left(h_{i}^{-1 / 2}+\widetilde{C} T^{1 / \alpha}\right) \mathbb{Z}_{i M}(\theta)+E\left(\left\langle R_{i} U_{i t}, \theta\right\rangle_{i} I_{\mathcal{E}_{M, t}^{c}} R_{i} U_{i t}\right)\right)\right\|_{i} \\
\leq & \left\|\theta_{1}-\theta_{2}\right\|_{i} C_{i}\left(1+h_{i}^{-1 / 2}\right)\left(T^{-1 / 2} C_{0} C_{i} \widetilde{C} T^{1 / \alpha}\left(h_{i}^{-1 / 2}+\widetilde{C} T^{1 / \alpha}\right)\left(J_{i}\left(p_{i}, 1\right)+T^{-1 / 2}\right)\right. \\
& \left.\times \sqrt{\log N+\log \log \left(T J_{i}\left(p_{i}, 1\right)\right)}+C_{i} h_{i}^{-1 / 2} E\left(\left(1+\left\|Z_{t}\right\|_{2}\right)^{\alpha}\right)^{2 / \alpha}\left(\frac{1}{\widetilde{C}^{\alpha} T} E\left(\left\|Z_{t}\right\|_{2}^{\alpha}\right)\right)^{1-2 / \alpha}\right) \\
\leq & c_{3}\left\|\theta_{1}-\theta_{2}\right\|_{i},
\end{aligned}
$$

where $c_{3}$ is a constant in $(0,1 / 2)$. Note that (A.8) holds also for $\theta_{1}=\theta_{2}$. The existence of such $c_{3}$ follows by condition $b_{N, p}=o_{P}(\sqrt{N} h)$.

In particular, letting $\theta_{2}=0$, one gets that for any $\theta_{1} \in \mathbb{B}\left(2 c_{2}\left(T h_{i}\right)^{-1 / 2}\right)$,

$$
\begin{aligned}
\left\|T_{2 i}\left(\theta_{1}\right)\right\|_{i} & \leq\left\|T_{2 i}\left(\theta_{1}\right)-T_{2 i}(0)\right\|_{i}+\left\|T_{2 i}(0)\right\|_{i} \\
& \leq c_{3}\left\|\theta_{1}\right\|_{i}+\left\|S_{i, M, \eta_{i}}\left(\theta_{\eta_{i}}\right)\right\|_{i} \\
& \leq 2 c_{2} c_{3}\left(T h_{i}\right)^{-1 / 2}+c_{2}\left(T h_{i}\right)^{-1 / 2}<2 c_{2}\left(T h_{i}\right)^{-1 / 2} .
\end{aligned}
$$

This implies that, with probability approaching one, $T_{2 i}$ is a contraction mapping from $\mathbb{B}\left(2 c_{2}\left(T h_{i}\right)^{-1 / 2}\right)$ to itself. By contraction mapping theorem, there exists uniquely a $\theta^{\prime \prime} \in \mathbb{B}\left(2 c_{2}\left(T h_{i}\right)^{-1 / 2}\right)$ such that $T_{2 i}\left(\theta^{\prime \prime}\right)=\theta^{\prime \prime}$, implying that $S_{i, M, \eta_{i}}\left(\theta_{\eta_{i}}+\theta^{\prime \prime}\right)=0$. Thus, $\widehat{\theta}_{i}=\theta_{\eta_{i}}+\theta^{\prime \prime}$ is the penalized MLE of $\ell_{i, M, \eta_{i}}$. This further shows that $\left\|\widehat{\theta}_{i}-\theta_{\eta_{i}}\right\|_{i} \leq 2 c_{2}\left(T h_{i}\right)^{-1 / 2}$. Combined with $\left\|\theta_{\eta_{i}}-\theta_{i 0}\right\|_{i}=$ $O\left(\eta_{i}^{1 / 2}+\left(N h_{i}\right)^{-1 / 2}\right)$, we have $\left\|\widehat{\theta}_{i}-\theta_{i 0}\right\|_{i}=O_{P}\left(\left(T h_{i}\right)^{-1 / 2}+\eta_{i}^{1 / 2}+\left(N h_{i}\right)^{-1 / 2}\right)$.

\section{A.2. Proofs in Section 3.3}

In this section, we prove Theorems 3.2, 3.3 and 3.4, and Corollary 3.6.

Proof of Theorem 3.2. Define

$$
S_{i, M}(\theta) \equiv-\frac{1}{T} \sum_{t=1}^{T}\left(Y_{i t}-\left\langle R_{i} U_{i t}, \theta\right\rangle_{i}\right) R_{i} U_{i t}
$$


and

$$
S_{i}(\theta) \equiv E\left\{S_{i, M}(\theta)\right\}=E\left\{-\frac{1}{T} \sum_{t=1}^{T}\left(Y_{i t}-\left\langle R_{i} U_{i t}, \theta\right\rangle_{i}\right) R_{i} U_{i t}\right\} .
$$

Recall $S_{i, M, \eta_{i}}=S_{i, M}+P_{i} \theta$ and $S_{i, M, \eta_{i}}^{\star}(\theta)=S_{i}(\theta)+P_{i} \theta$. Denote $\theta_{i}=\widehat{\theta}_{i}-\theta_{i 0}$. Since $S_{i, M, \eta_{i}}\left(\widehat{\theta}_{i}\right)=0$, we have $S_{i, M, \eta_{i}}\left(\theta_{i}+\theta_{i 0}\right)=0$. Therefore,

$$
\begin{aligned}
& \left\|S_{i, M}\left(\theta_{i}+\theta_{i 0}\right)-S_{i}\left(\theta_{i}+\theta_{i 0}\right)-\left(S_{i, M}\left(\theta_{i 0}\right)-S_{i}\left(\theta_{i 0}\right)\right)\right\|_{i} \\
= & \left\|S_{i, M, \eta_{i}}\left(\theta_{i}+\theta_{i 0}\right)-S_{i, M, \eta_{i}}^{\star}\left(\theta_{i}+\theta_{i 0}\right)-\left(S_{i, M, \eta_{i}}\left(\theta_{i 0}\right)-S_{i, M, \eta_{i}}^{\star}\left(\theta_{i 0}\right)\right)\right\|_{i} \\
= & \left\|S_{i, M, \eta_{i}}^{\star}\left(\theta_{i}+\theta_{i 0}\right)+S_{i, M, \eta_{i}}\left(\theta_{i 0}\right)-S_{i, M, \eta_{i}}^{\star}\left(\theta_{i 0}\right)\right\|_{i} \\
= & \left\|D S_{i, M, \eta_{i}}^{\star}\left(\theta_{i 0}\right) \theta_{i}+S_{i, M, \eta_{i}}\left(\theta_{i 0}\right)\right\|_{i} \\
= & \left\|\theta_{i}+S_{i, M, \eta_{i}}\left(\theta_{i 0}\right)\right\|_{i} .
\end{aligned}
$$

Consider an event $B_{i, M}=\left\{\|\theta\|_{i} \leq r_{i, M} \equiv C_{B}\left(\left(T h_{i}\right)^{-1 / 2}\right)+\eta_{i}^{1 / 2}+\left(N h_{i}\right)^{-1 / 2}\right\}$. For some $C_{B}$ large enough, $B_{i, M}$ has probability approaching one. Let $d_{i, M}=C_{i} r_{i, M}\left(1+h_{i}^{-1 / 2}\right)$, where $C_{i}$ is defined in lemma A.2. We have $d_{i, M}=o(1)$. For any $\theta \in \Theta_{i}$, we further define $\bar{\theta}=(\bar{\beta}, \bar{g})=d_{i, M}^{-1} \theta / 2$, where $\bar{\beta}=d_{i, M}^{-1} \beta / 2$ and $\bar{g}=d_{i, M}^{-1} g / 2$. Then, on event $B_{i, M}$, we have

$$
\|\bar{\theta}\|_{i, \text { sup }} \leq C_{i}\left(1+h_{i}^{-1 / 2}\right)\|\bar{\theta}\|_{i}=C_{i}\left(1+h_{i}^{-1 / 2}\right) d_{i, M}^{-1}\|\theta\|_{i} / 2 \leq \frac{1}{2} .
$$

Meanwhile,

$$
\|\bar{g}\|_{H_{i}}^{2}=\frac{d_{i, M}^{-2}}{4} \eta_{i}^{-1}\left(\eta_{i}\|g\|_{H_{i}}^{2}\right) \leq \frac{d_{i, M}^{-2}}{4} \eta_{i}^{-1}\|\theta\|_{i}^{2} \leq \frac{d_{i, M}^{-2}}{4} \eta_{i}^{-1} r_{i, M}^{2} \leq C_{i}^{-2} h_{i} \eta_{i}^{-1} .
$$

Let $p_{i}=C_{i}^{-1}\left(h_{i} \eta_{i}^{-1}\right)^{1 / 2}$. Then $\|\bar{g}\|_{H_{i}} \leq p_{i}$. Therefore $\bar{\theta} \in \mathcal{G}_{i}\left(p_{i}\right)$. Since $\left(\eta_{i} h_{i}^{-1}\right) \rightarrow \infty$ as $(N, T) \rightarrow$ $\infty, p_{i}>1$ in general.

Recall $\mathcal{E}_{M, t}=\left\{\left\|Z_{t}\right\|_{2} \leq \widetilde{C} T^{1 / \alpha}\right\}$, as defined in the proof of Theorem 3.1. Let

$$
\psi_{i, M, t}^{d}\left(U_{i t} ; \bar{\theta}\right)=\frac{\left\langle R_{i} U_{i t}, \theta\right\rangle_{i} I_{\mathcal{E}_{M, t}}}{2 d_{M} \widetilde{C} C_{i} T^{1 / \alpha}\left(h_{i}^{-1 / 2}+\widetilde{C} T^{1 / \alpha}\right)}, \theta \in \Theta_{i}
$$

Following the proof of Theorem 3.1, on $\mathcal{E}_{M}$, for any $\theta_{1}=\left(\beta_{1}, g_{1}\right), \theta_{2}=\left(\beta_{2}, g_{2}\right) \in \Theta_{i}$, we have

$$
\left\|\left(\psi_{i, M, t}^{d}\left(U_{i t} ; \bar{\theta}_{1}\right)-\psi_{i M t}^{d}\left(U_{i t} ; \bar{\theta}_{2}\right)\right) R_{i} U_{i t}\right\|_{i} \leq\left\|\bar{\theta}_{1}-\bar{\theta}_{2}\right\|_{i, \text { sup }}
$$

Define

$$
\mathbb{Z}_{i M}^{d}(\bar{\theta})=\frac{1}{\sqrt{T}} \sum_{t=1}^{T}\left[\psi_{i, M, t}^{d}\left(U_{i t} ; \bar{\theta}\right) R_{i} U_{i t}-E\left(\psi_{i, M, t}^{d}\left(U_{i t} ; \bar{\theta}\right) R_{i} U_{i t}\right)\right] .
$$

It follows from Proposition A.2 that, with probability approaching one,

$$
\sup _{\theta \in \mathcal{G}_{i}\left(p_{i}\right)} \frac{\sqrt{T}\left\|Z_{i M}^{d}(\bar{\theta})\right\|_{i}}{\sqrt{T} J_{i}\left(p_{i},\|\bar{\theta}\|_{i, \text { sup }}\right)+1} \leq C_{0} \sqrt{\log N+\log \log \left(T J_{i}\left(p_{i}, 1\right)\right)} .
$$


Therefore

$$
\begin{aligned}
& \left\|\theta_{i}+S_{i, M, \eta_{i}}\left(\theta_{i 0}\right)\right\|_{i} \\
= & \left\|S_{i, M}\left(\theta_{i}+\theta_{i 0}\right)-S_{i}\left(\theta_{i}+\theta_{i 0}\right)-\left(S_{i, M}\left(\theta_{i 0}\right)-S_{i}\left(\theta_{i 0}\right)\right)\right\|_{i} \\
= & \left\|\frac{1}{T} \sum_{t=1}^{T}\left[\left\langle R_{i} U_{i t}, \theta_{i}\right\rangle_{i} R_{i} U_{i t}-E\left\{\left\langle R_{i} U_{i t}, \theta_{i}\right\rangle_{i} R_{i} U_{i t}\right\}\right]\right\|_{i} \\
= & \left\|\frac{1}{T} \sum_{t=1}^{T}\left[\left\langle R_{i} U_{i t}, \theta_{i}\right\rangle_{i} I_{\mathcal{E}_{M, t}} R_{i} U_{i t}-E\left(\left\langle R_{i} U_{i t}, \theta_{i}\right\rangle_{i} I_{\mathcal{E}_{M, t}} R_{i} U_{i t}\right)\right]-E\left(\left\langle R_{i} U_{i t}, \theta_{i}\right\rangle_{i} I_{\mathcal{E}_{M, t}^{c}} R_{i} U_{i t}\right)\right\|_{i} \\
= & \left\|2 d_{M} \widetilde{C} C_{i} T^{1 / \alpha-1}\left(h_{i}^{-1 / 2}+\widetilde{C} T^{1 / \alpha}\right)\left(\sqrt{T} Z_{i, M}^{d}(\bar{\theta})\right)-E\left(\left\langle R_{i} U_{i t}, \theta_{i}\right\rangle_{i} I_{\mathcal{E}_{M, t}^{c}} R_{i} U_{i t}\right)\right\|_{i} \\
\leq & 2 d_{M} C_{0} \widetilde{C} C_{i} T^{1 / \alpha-1}\left(h_{i}^{-1 / 2}+\widetilde{C} T^{1 / \alpha}\right)\left(\sqrt{T} J_{i}\left(p_{i},\|\bar{\theta}\|_{i, \mathrm{sup}}\right)+1\right) \sqrt{\log N+\log \log \left(T J_{i}\left(p_{i}, 1\right)\right)} \\
& +C_{i} h_{i}^{-1 / 2} E\left(\left(1+\left\|Z_{t}\right\|_{2}\right)^{\alpha}\right)^{2 / \alpha}\left(\frac{1}{\widetilde{C}^{\alpha} T} E\left(\left\|Z_{t}\right\|_{2}^{\alpha}\right)\right)^{1-2 / \alpha} .
\end{aligned}
$$

Proof of Theorem 3.3. Define $\widehat{\theta}_{i}^{h}=\left(\widehat{\beta}_{i}, h_{i}^{1 / 2} \widehat{g}_{i}\right), \theta_{i 0}^{* h}=\left(\beta_{i 0}^{\star}, h_{i}^{1 / 2} g_{i 0}^{\star}\right)$, and $R_{i}^{h} u=\left(H_{u}^{(i)}, h_{i}^{1 / 2} T_{u}^{(i)}\right)$, where $\theta_{i 0}^{\star}=\left(i d-P_{i}\right) \theta_{i 0}$. From Theorem 3.2, we have

$$
\left\|\widehat{\theta}_{i}-\theta_{i 0}+S_{i, M, \eta_{i}}\left(\theta_{i 0}\right)\right\|_{i}=O_{P}\left(a_{M}\right)
$$

Since

$$
S_{i, M, \eta_{i}}\left(\theta_{i 0}\right)=-\frac{1}{T} \sum_{t=1}^{T}\left(Y_{i t}-\left\langle R_{i} U_{i t}, \theta_{i 0}\right\rangle_{i}\right) R_{i} U_{i t}+P_{i} \theta_{i 0}=-\frac{1}{T} \sum_{t=1}^{T} e_{i t} R_{i} U_{i t}+P_{i} \theta_{i 0}
$$

Theorem 3.2 can be re-written as

$$
\left\|\widehat{\theta}_{i}-\theta_{i 0}^{\star}-\frac{1}{T} \sum_{t=1}^{T} e_{i t} R_{i} U_{i t}\right\|_{i}=O_{P}\left(a_{M}\right) .
$$

It implies $\left\|\widehat{\beta}_{i}-\beta_{i 0}^{\star}-\frac{1}{T} \sum_{t=1}^{T} e_{i t} H_{i t}^{(i)}\right\|_{2}=O_{P}\left(a_{M}\right)$. Further, we define $R e m=\widehat{\theta}_{i}-\theta_{i 0}^{\star}-\frac{1}{T} \sum_{t=1}^{T} e_{i t} R_{i} U_{i t}$ and $\operatorname{Rem}^{h}=\widehat{\theta}_{i}^{h}-\theta_{i 0}^{* h}-\frac{1}{T} \sum_{t=1}^{T} e_{i t} R_{i}^{h} U_{i t}$. Then

$$
\begin{aligned}
\left\|\operatorname{Rem}^{h}-h_{i}^{1 / 2} \operatorname{Rem}\right\|_{i} & =\left\|\left(\left(1-h_{i}^{1 / 2}\right)\left(\widehat{\beta}_{i}-\beta_{i 0}^{\star}-\frac{1}{T} \sum_{t=1}^{T} e_{i t} H_{U_{i t}}^{(i)}\right), 0\right)\right\|_{i} \\
& \left.\leq\left(1-h_{i}^{1 / 2}\right) O\left(\| \widehat{\beta}_{i}-\beta_{i 0}^{\star}-\frac{1}{T} \sum_{t=1}^{T} e_{i t} H_{U_{i t}}^{(i)}\right) \|_{2}\right)=O_{P}\left(a_{M}\right) .
\end{aligned}
$$

Therefore, $\left\|\operatorname{Rem}^{h}\right\|_{i} \leq\left\|\operatorname{Rem}^{h}-h_{i}^{1 / 2} \operatorname{Rem}\right\|_{i}+\left\|h_{i}^{1 / 2} \operatorname{Rem}\right\|_{i}=O_{P}\left(a_{M}\right)$.

The idea is to employ the Cramér-Wold device. For any $z$, we will obtain the limiting distribution of $T^{1 / 2} z^{\prime}\left(\widehat{\beta}_{i}-\beta_{i 0}^{\star}\right)+\left(T h_{i}\right)^{1 / 2}\left(\widehat{g}_{i}\left(x_{0}\right)-g_{i 0}^{\star}\left(x_{0}\right)\right)$, which is $T^{1 / 2}\left\langle R_{i} u, \widehat{\theta}_{i}^{h}-\theta_{i 0}^{* h}\right\rangle_{i}$ by Proposition 2.1 , where $u=\left(x_{0}, z\right)$. 
Since $T^{1 / 2} h^{-1 / 2} a_{M}=o(1)$, we have

$$
\begin{aligned}
& \left|T^{1 / 2}\left\langle R_{i} u, \widehat{\theta}_{i}^{h}-\theta_{i 0}^{* h}-\frac{1}{T} \sum_{t=1}^{T} e_{i t} R_{i}^{h} U_{i t}\right\rangle_{i}\right| \\
& \leq T^{1 / 2}\left\|R_{i} u\right\|_{i}\left\|R e m^{h}\right\|_{i} \\
& =O_{P}\left(T^{1 / 2} h^{-1 / 2} a_{M}\right)=o_{P}(1) .
\end{aligned}
$$

Then, to find the limiting distribution of $T^{1 / 2}\left\langle R_{i} u, \widehat{\theta}_{i}^{h}-\theta_{i 0}^{* h}\right\rangle_{i}$, we only need to find the limiting distribution of $T^{1 / 2}\left\langle R_{i} u, \frac{1}{T} \sum_{t=1}^{T} e_{i t} R_{i}^{h} U_{i t}\right\rangle_{i}=T^{-1 / 2} \sum_{t=1}^{T} e_{i t}\left(z^{\prime} H_{U_{i t}^{(i)}}+h^{1 / 2} T_{U_{i t}}^{(i)}\left(x_{0}\right)\right)$. Next we will use CLT to find its limiting distribution.

Define $L\left(U_{i t}\right)=z^{\prime} H_{U_{i t}}^{(i)}+h^{1 / 2} T_{U_{i t}}^{(i)}\left(x_{0}\right)$. Since $\epsilon_{i t}$ and $v_{i t}$ are i.i.d. across $t$, we have

$$
\begin{aligned}
s_{T}^{2} & =\operatorname{Var}\left(\sum_{t=1}^{T} e_{i t}\left(z^{\prime} H_{U_{i t}}^{(i)}+h^{1 / 2} T_{U_{i t}}^{(i)}\left(x_{0}\right)\right)\right) \\
& =T \cdot \operatorname{Var}\left(e_{i t} L\left(U_{i t}\right)\right)+\sum_{t_{1} \neq t_{2}}^{T} \operatorname{Cov}\left(e_{i t_{1}} L\left(U_{i t_{1}}\right), e_{i t_{2}} L\left(U_{i t_{2}}\right)\right) \\
& =T \cdot E\left\{e_{i t}^{2} L\left(U_{i t}\right)^{2}\right\}-T \cdot E\left\{e_{i t} L\left(U_{i t}\right)\right\}^{2}+\sum_{t_{1} \neq t_{2}}^{T} \operatorname{Cov}\left(e_{i t_{1}} L\left(U_{i t_{1}}\right), e_{i t_{2}} L\left(U_{i t_{2}}\right)\right) .
\end{aligned}
$$

For the first term,

$$
E\left\{e_{i t}^{2} L\left(U_{i t}\right)^{2}\right\}=E\left\{\left(\epsilon_{i t}-\Delta_{i} \bar{v}_{t}\right)^{2} L\left(U_{i t}\right)^{2}\right\}=\sigma_{\epsilon}^{2} E\left\{L\left(U_{i t}\right)^{2}\right\}+E\left\{\left(\Delta_{i} \bar{v}_{t}\right)^{2} L\left(U_{i t}\right)^{2}\right\} .
$$

From Cauchy-Schwarz and Hölder's inequality, we can show that

$$
\begin{aligned}
E\left\{\left(\Delta_{i} \bar{v}_{t}\right)^{2} L\left(U_{i t}\right)^{2}\right\} & \leq\left\|\Delta_{i}\right\|_{2}^{2} E\left\{\left\|\bar{v}_{t}\right\|_{2}^{2} L\left(U_{i t}\right)^{2}\right\} \\
& \leq\left\|\Delta_{i}\right\|_{2}^{2}\left(E\left\{\left\|\bar{v}_{t}\right\|_{2}^{\alpha}\right\}\right)^{2 / \alpha}\left(E\left\{\left|L\left(U_{i t}\right)\right|^{\frac{2 \alpha}{\alpha-2}}\right\}\right)^{\frac{\alpha-2}{\alpha}} .
\end{aligned}
$$

We next will find the upper bound of $\left|L\left(U_{i t}\right)\right|$.

$$
\begin{aligned}
L\left(U_{i t}\right)= & z^{\prime} H_{U_{i t}}^{(i)}+h^{1 / 2} T_{U_{i t}}^{(i)}\left(x_{0}\right) \\
= & z^{\prime}\left(\Omega_{i}+\Sigma_{i}\right)^{-1} Z_{t}-z^{\prime}\left(\Omega_{i}+\Sigma_{i}\right)^{-1} A_{i}\left(X_{i t}\right)+h_{i}^{1 / 2} K_{X_{i t}}^{(i)}\left(x_{0}\right) \\
& -h_{i}^{1 / 2} A_{i}^{\prime}\left(x_{0}\right)\left(\Omega_{i}+\Sigma_{i}\right)^{-1} Z_{t}+h_{i}^{1 / 2} A_{i}^{\prime}\left(x_{0}\right)\left(\Omega_{i}+\Sigma_{i}\right)^{-1} A_{i}\left(X_{i t}\right) .
\end{aligned}
$$


By the proof of lemma A.2, we have

$$
\begin{aligned}
\left|z^{\prime}\left(\Omega_{i}+\Sigma_{i}\right)^{-1} Z_{t}\right| & \leq\left|c_{1}^{-1} z^{\prime} Z_{t}\right| \leq c_{1 z}\left\|Z_{t}\right\|_{2} \\
\left|z^{\prime}\left(\Omega_{i}+\Sigma_{i}\right)^{-1} A_{i}\left(X_{i t}\right)\right| & =\left|z^{\prime}\left(\Omega_{i}+\Sigma_{i}\right)^{-1 / 2}\left(\Omega_{i}+\Sigma_{i}\right)^{-1 / 2} A_{i}\left(X_{i t}\right)\right| \\
& \leq \sqrt{z^{\prime}\left(\Omega_{i}+\Sigma_{i}\right)^{-1} z} \sqrt{A_{i}^{\prime}\left(X_{i t}\right)\left(\Omega_{i}+\Sigma_{i}\right)^{-1} A_{i}\left(X_{i t}\right)} \\
& \leq c_{2 z} h_{i}^{-1 / 2}, \\
\left|A_{i}^{\prime}\left(x_{0}\right)\left(\Omega_{i}+\Sigma_{i}\right)^{-1} Z_{t}\right| & \leq c_{3 z} h_{i}^{-1 / 2}\left\|Z_{t}\right\|_{2}, \\
\left|K_{X_{i t}}^{(i)}\left(x_{0}\right)\right| & \leq C_{\varphi, i}^{2} h_{i}^{-1}, \\
\left|A_{i}^{\prime}\left(x_{0}\right)\left(\Omega_{i}+\Sigma_{i}\right)^{-1} A_{i}\left(X_{i t}\right)\right| & \leq c_{1}^{-1} C_{\varphi, i}^{2} C_{G_{i}}^{2} h_{i}^{-1},
\end{aligned}
$$

where $c_{1 z}=c_{1}^{-1}\|z\|_{2}, c_{2 z}=c_{1}^{-1} C_{\varphi, i} C_{G_{i}}\|z\|_{2}$, and $c_{3 z}=c_{1}^{-1} C_{\varphi, i} C_{G_{i}}$. Combine all these inequality together, we have

$$
\left|L\left(U_{i t}\right)\right| \leq c_{4 z}\left\|Z_{t}\right\|_{2}+c_{5 z} h_{i}^{-1 / 2}
$$

where $c_{4 z}=c_{1 z}+c_{3 z}$ and $c_{5 z}=c_{2 z}+C_{\varphi, i}^{2}+c_{1}^{-1} C_{\varphi, i}^{2} C_{G_{i}}^{2}$. Therefore, there exists a constant $c_{6 z}$, such that

$$
\begin{aligned}
& E\left\{\left|L\left(U_{i t}\right)\right|^{\frac{2 \alpha}{\alpha-2}}\right\}=E\left\{\left|c_{4 z}\left\|Z_{t}\right\|_{2}+c_{5 z} h_{i}^{-1 / 2}\right|^{\frac{2 \alpha}{\alpha-2}}\right\} \\
& \leq c_{6 z}\left(E\left\{\left\|Z_{t}\right\|_{2}^{\frac{2 \alpha}{\alpha-2}}\right\}+h_{i}^{-\frac{\alpha}{\alpha-2}}\right) \\
& \leq c_{6 z}\left(E\left\{\left\|Z_{t}\right\|_{2}^{\alpha}\right\}^{\frac{2}{\alpha-2}}+h_{i}^{-\frac{\alpha}{\alpha-2}}\right) \text {. }
\end{aligned}
$$

Since $Z_{t}=\left(f_{1 t}^{\prime}, \bar{X}_{t}^{\prime}\right)^{\prime}$ and $\bar{X}_{t}=\bar{\Gamma}_{1}^{\prime} f_{1 t}+\bar{\Gamma}_{2}^{\prime} f_{2 t}+\bar{v}_{t}$, we have

$$
\begin{aligned}
E\left\{\left\|Z_{t}\right\|_{2}^{\alpha}\right\} & =E\left\{\left(\left\|f_{1 t}\right\|_{2}^{2}+\left\|\bar{X}_{t}\right\|_{2}^{2}\right)^{\alpha / 2}\right\} \\
& \leq c_{7}\left(E\left\{\left\|f_{1 t}\right\|_{2}^{\alpha}\right\}+E\left\{\left\|\bar{X}_{t}\right\|_{2}^{\alpha}\right\}\right) \\
& \leq c_{8}\left(E\left\{\left\|f_{1 t}\right\|_{2}^{\alpha}\right\}+E\left\{\left\|f_{1 t}\right\|_{2}^{\alpha}\right\}+E\left\{\left\|f_{2 t}\right\|_{2}^{\alpha}\right\}+E\left\{\left\|\bar{v}_{t}\right\|_{2}^{\alpha}\right\}\right),
\end{aligned}
$$

where $c_{7}$ and $c_{8}$ are constants. By Assumption A2, $E\left\{\left\|f_{1 t}\right\|_{2}^{\alpha}\right\}, E\left\{\left\|f_{2 t}\right\|_{2}^{\alpha}\right\}$, and $E\left\{\left|v_{i t}\right|^{\alpha}\right\}$ are 
finite. Using Marcinkiewicz and Zygmund inequality in Shao (2003), we can show that

$$
\begin{aligned}
E\left\{\left\|\bar{v}_{t}\right\|_{2}^{\alpha}\right\} & =\frac{1}{N^{\alpha}} E\left\{\left(\sum_{l=1}^{d}\left(\sum_{i=1}^{N} v_{i l t}\right)^{2}\right)^{\alpha / 2}\right\} \\
& \leq c_{9} \frac{1}{N^{\alpha}} \sum_{l=1}^{d} E\left\{\left(\left(\sum_{i=1}^{N} v_{i l t}\right)^{2}\right)^{\alpha / 2}\right\} \\
& =c_{9} \frac{1}{N^{\alpha}} \sum_{l=1}^{d} E\left\{\left(\sum_{i=1}^{N} v_{i l t}\right)^{\alpha}\right\} \\
& \leq c_{10} \frac{d}{N^{\alpha}} \frac{1}{N^{1-\alpha / 2}} \sum_{i=1}^{N} E\left\{v_{i l t}^{\alpha}\right\} \\
& =O_{P}\left(\frac{1}{N^{\alpha / 2}}\right)
\end{aligned}
$$

where $c_{9}$ and $c_{10}$ are two constants. As $N \rightarrow \infty, E\left\{\left\|Z_{t}\right\|_{2}^{\alpha}\right\}=O_{P}(1)$ and $E\left\{\left|L\left(U_{i t}\right)\right|^{\frac{2 \alpha}{\alpha-2}}\right\}=$ $O_{P}\left(h_{i}^{-\frac{\alpha}{\alpha-2}}\right)$. Thus, we have

$$
E\left\{\left(\Delta_{i} \bar{v}_{t}\right)^{2} L\left(U_{i t}\right)^{2}\right\} \leq\left\|\Delta_{i}\right\|_{2}^{2}\left(E\left\{\left\|\bar{v}_{t}\right\|_{2}^{\alpha}\right\}\right)^{2 / \alpha}\left(E\left\{\mid L\left(U_{i t}\right)^{\frac{2 \alpha}{\alpha-2}}\right\}\right)^{\frac{\alpha-2}{\alpha}}=O_{P}\left(\frac{1}{N h_{i}}\right)
$$

By assumption $\left(N h_{i}\right)^{-1}=o_{P}(1)$, we have $E\left\{\left(\Delta_{i} \bar{v}_{t}\right)^{2} L\left(U_{i t}\right)^{2}\right\}=o_{P}(1)$.

Next, we will find the order of $E\left\{L\left(U_{i t}\right)^{2}\right\}$. As it is shown in Cheng and Shang (2015), as $\eta_{i} \rightarrow 0$ and $T \rightarrow \infty, E\left(\left|L\left(U_{i t}\right)\right|^{2}\right) \rightarrow \alpha_{x_{0}}^{2}+2\left(z+\beta_{x_{0}}\right)^{\prime} \Omega_{i}^{-1} \alpha_{x_{0}}+\left(z+\beta_{x_{0}}\right)^{\prime} \Omega_{i}^{-1}\left(z+\beta_{x_{0}}\right)$ for any given $z$.

From above derivatives, we have found the leading term of the first term in equation (A.15). Now, we turn to the second term. It is straightforward to obtain that

$$
E\left\{e_{i t} L\left(U_{i t}\right)\right\}^{2}=E\left\{\left(\epsilon_{i t}-\Delta_{i} \bar{v}_{t}\right) L\left(U_{i t}\right)\right\}^{2} \leq\left\|\Delta_{i}\right\|_{2}^{2} E\left\{\left\|\bar{v}_{t}\right\|_{2}^{2}\right\} E\left\{L\left(U_{i t}\right)^{2}\right\},
$$

where $E\left\{\left\|\bar{v}_{t}\right\|_{2}^{2}\right\} \leq E\left\{\left\|\bar{v}_{t}\right\|_{2}^{\alpha}\right\}^{2 / \alpha}=O_{P}(1 / N)$ and $E\left\{L\left(U_{i t}\right)^{2}\right\}=O_{P}(1)$. So $E\left\{e_{i t} L\left(U_{i t}\right)\right\}^{2}=$ $O_{P}(1 / N)$. So the second term is of a smaller order than the first term. 
For the last term of equation (A.15), we can show that

$$
\begin{aligned}
& \sum_{t_{1} \neq t_{2}}^{T} \operatorname{Cov}\left(e_{i t_{1}} L\left(U_{i t_{1}}\right), e_{i t_{2}} L\left(U_{i t_{2}}\right)\right) \\
\leq & \sum_{t_{1} \neq t_{2}}^{T}\left|\operatorname{Cov}\left(\Delta_{i} \bar{v}_{t_{1}} L\left(U_{i t_{1}}\right), \Delta_{i} \bar{v}_{t_{2}} L\left(U_{i t_{2}}\right)\right)\right| \\
\leq & 8 \sum_{t_{1} \neq t_{2}}^{T} \alpha\left(\left|t_{1}-t_{2}\right|\right)^{1-4 / \alpha} E\left\{\left|\Delta_{i} \bar{v}_{t_{1}} L\left(U_{i t_{1}}\right)\right|^{\alpha / 2}\right\}^{4 / \alpha} \\
\leq & 8 \cdot 2^{1-4 / \alpha} \sum_{t_{1}=1}^{T} \sum_{t_{2}=1, t_{2} \neq t_{1}}^{T} \phi\left(\left|t_{1}-t_{2}\right|\right)^{1-4 / \alpha} E\left\{\left|\Delta_{i} \bar{v}_{t_{1}} L\left(U_{i t_{1}}\right)\right|^{\alpha / 2}\right\}^{4 / \alpha},
\end{aligned}
$$

where $\alpha\left(\left|t_{1}-t_{2}\right|\right)$ and $\phi\left(\left|t_{1}-t_{2}\right|\right)$ are the $\alpha-$ mixing and $\phi-$ mixing coefficients for $\Delta_{i} \bar{v}_{t} L\left(U_{i t}\right)$. We have $2 \cdot \alpha\left(\left|t_{1}-t_{2}\right|\right)<\phi\left(\left|t_{1}-t_{2}\right|\right)$. The second inequality is from Proposition 2.5 in Fan and Yao (2003). Similar to equation (A.16), we can show that $E\left\{\mid \Delta_{i} \bar{v}_{t_{1}} L\left(U_{i t_{1}}\right)^{\alpha}\right\}=O_{P}\left(\left(N h_{i}\right)^{-\alpha / 2}\right)$. So $E\left\{\left|\Delta_{i} \bar{v}_{t_{1}} L\left(U_{i t_{1}}\right)\right|^{\alpha / 2}\right\}^{4 / \alpha}=O_{P}\left(\frac{1}{N h_{i}}\right)$. From Assumption A2, we have $\sum_{t_{2}=1, t_{2} \neq t_{1}}^{\infty} \phi\left(\left|t_{1}-t_{2}\right|\right)^{1-4 / \alpha}<$ $\infty$. Thus,

$$
\sum_{t_{1} \neq t_{2}}^{T} \operatorname{Cov}\left(e_{i t_{1}} L\left(U_{i t_{1}}\right), e_{i t_{2}} L\left(U_{i t_{2}}\right)\right)=O_{P}\left(\frac{T}{N h_{i}}\right) .
$$

Again, the third term of equation (A.15) is of a smaller order than the first term.

To combine all above equations together, we have

$$
\frac{1}{T} s_{T}^{2} \rightarrow \sigma_{s}^{2} \quad \text { or } \quad \frac{1}{T} s_{T}^{2} \rightarrow\left(z^{\prime}, 1\right) \Psi^{\star}\left(z^{\prime}, 1\right)^{\prime},
$$

where $\sigma_{s}^{2}=\sigma_{\epsilon}^{2}\left(\alpha_{x_{0}}^{2}+2\left(z+\beta_{x_{0}}\right)^{\prime} \Omega_{i}^{-1} \alpha_{x_{0}}+\left(z+\beta_{x_{0}}\right)^{\prime} \Omega_{i}^{-1}\left(z+\beta_{x_{0}}\right)\right)$.

In order to use the CLT with mixing conditions, we need to show $E\left\{\left|e_{i t} L\left(U_{i t}\right)\right|^{\alpha / 2}\right\}$ is finite.

$$
\begin{aligned}
E\left\{\left|e_{i t} L\left(U_{i t}\right)\right|^{\alpha / 2}\right\} & \leq E\left\{\left|e_{i t}\right|^{\alpha}\right\}^{1 / 2} E\left\{\left|L\left(U_{i t}\right)\right|^{\alpha}\right\}^{1 / 2} \\
& \leq c_{6 z} E\left\{\left|e_{i t}\right|^{\alpha}\right\}^{1 / 2} E\left\{\left\|Z_{t}\right\|_{2}^{\alpha}+h_{i}^{-\alpha / 2}\right\}^{1 / 2}
\end{aligned}
$$

Since $E\left\{\left\|Z_{t}\right\|_{2}^{\alpha}\right\}=O_{P}(1)$ and $E\left\{\left|e_{i t}\right|^{\alpha}\right\} \leq \infty$, then $E\left\{\left|e_{i t} L\left(U_{i t}\right)\right|^{\alpha / 2}\right\} \leq \infty$. From the above proof, we have $E\left\{e_{i t} L\left(U_{i t}\right)\right\}^{2}=O_{P}(1 / N)$, which implies

$$
E\left\{e_{i t} L\left(U_{i t}\right)\right\}=O_{P}(1 / \sqrt{N}) .
$$

Then $E\left\{\left|e_{i t} L\left(U_{i t}\right)-E\left\{e_{i t} L\left(U_{i t}\right)\right\}\right|^{\alpha / 2}\right\}<\infty$. Since $\phi$-mixing condition is stronger than $\alpha$-mixing, by the Theorem 2.21 of Fan and Yao (2003), we have

$$
\frac{1}{\sqrt{T}}\left(\sum_{t=1}^{T}\left(e_{i t} L\left(U_{i t}\right)-E\left\{e_{i t} L\left(U_{i t}\right)\right\}\right)\right) \stackrel{d}{\rightarrow} N\left(0, \sigma_{s}^{2}\right) .
$$


Proof of Theorem 3.4. Notice

$$
\theta_{i 0}-\theta_{i 0}^{\star}=P_{i} \theta_{i 0}=\left(\begin{array}{c}
-\left(\Omega_{i}+\Sigma_{i}\right)^{-1} V_{i}\left(G_{i}, W_{i} g_{i 0}\right) \\
W_{i} g_{i 0}+A_{i}^{\prime}\left(\Omega_{i}+\Sigma_{i}\right)^{-1} V_{i}\left(G_{i}, W_{i} g_{i 0}\right)
\end{array}\right) .
$$

Hence the result of the theorem holds if we can show that, for any $x \in \mathcal{X}_{i}$,

$$
\begin{gathered}
\sqrt{T} E\left(e_{i t} U_{i t}^{(i)}\right)=o_{P}(1), \\
\sqrt{T h_{i}} E\left(e_{i t} H_{i t}^{(i)}(x)\right), \\
\sqrt{T}\left(\Omega_{i}+\Sigma_{i}\right)^{-1} V_{i}\left(G_{i}, W_{i} g_{i 0}\right)=o_{P}(1), \\
\sqrt{T h_{i}} A_{i}^{\prime}(x)\left(\Omega_{i}+\Sigma_{i}\right)^{-1} V_{i}\left(G_{i}, W_{i} g_{i 0}\right)=o_{P}(1), \\
\alpha_{x}=\beta_{x}=0, \\
\lim _{T \rightarrow \infty} \sqrt{T h_{i}} W_{i} g_{i 0}(x)=0 .
\end{gathered}
$$

First by (A.17), we can see that the follow hold true:

$$
\begin{aligned}
\sqrt{T} E\left(e_{i t} U_{i t}^{(i)}\right) & =O_{P}(\sqrt{T / N})=o_{P}(1), \\
\sqrt{T h_{i}} E\left(e_{i t} H_{i t}^{(i)}(x)\right) & =O_{P}(\sqrt{T / N})=o_{P}(1) .
\end{aligned}
$$

Similar to Shang and Cheng (2013), we have

$$
W_{i} \varphi_{\nu}^{(i)}=\frac{\eta_{i} \rho_{\nu}^{(i)}}{1+\eta_{i} \rho_{\nu}^{(i)}} \varphi_{\nu}^{(i)}, \nu \geq 1
$$

Now we see from A3 and (A.18)

$$
V_{i}\left(G_{i, k}, W_{i} g_{i 0}\right)=\sum_{\nu \geq 1} V_{i}\left(G_{i, k}, \varphi_{\nu}^{(i)}\right) V_{i}\left(g_{i 0}, \varphi_{\nu}^{(i)}\right) \frac{\eta_{i} \rho_{\nu}^{(i)}}{1+\eta_{i} \rho_{\nu}^{(i)}} .
$$

So by Cauchy's inequality,

$$
\begin{aligned}
\left|V_{i}\left(G_{i, k}, W_{i} g_{i 0}\right)\right|^{2} & \leq \sum_{\nu \geq 1}\left|V_{i}\left(G_{i, k}, \varphi_{\nu}^{(i)}\right)\right|^{2} \frac{\eta_{i} \rho_{\nu}^{(i)}}{1+\eta_{i} \rho_{\nu}^{(i)}} \sum_{\nu \geq 1}\left|V_{i}\left(g_{i 0}, \varphi_{\nu}^{(i)}\right)\right|^{2} \frac{\eta_{i} \rho_{\nu}^{(i)}}{\left(1+\eta_{i} \rho_{\nu}^{(i)}\right)} \\
& \leq \eta_{i} \text { const } \sum_{\nu \geq 1}\left|V_{i}\left(G_{i, k}, \varphi_{\nu}^{(i)}\right)\right|^{2} \frac{\eta_{i} \rho_{\nu}^{(i)}}{1+\eta_{i} \rho_{\nu}^{(i)}} \\
& \leq \eta_{i} \text { const } \sum_{\nu \geq 1}\left|V_{i}\left(G_{i, k}, \varphi_{\nu}^{(i)}\right)\right|^{2} k_{\nu} \frac{\eta_{i} \rho_{\nu}^{(i)}}{\left(1+\eta_{i} \rho_{\nu}^{(i)}\right) k_{\nu}} \\
& \leq \eta_{i} \text { const },
\end{aligned}
$$

For $\left\|A_{i, k}\right\|_{\text {sup }}$, by definition,

$$
A_{i, k}(x)=\left\langle A_{i, k}, K_{x}^{(i)}\right\rangle_{\star, i} \leq V_{i}\left(G_{i, k}, K_{x}^{(i)}\right)=\sum_{\nu \geq 1} \frac{V_{i}\left(G_{i, k}, \varphi_{\nu}^{(i)}\right)}{1+\eta_{i} \rho_{\nu}^{(i)}} \varphi_{\nu}^{(i)}(x) .
$$


By boundedness condition of $\varphi_{\nu}^{(i)}$ (Assumption A3) and Cauchy's inequality, we have

$$
\begin{aligned}
\left|A_{i, k}(x)\right|^{2} & \leq \sum_{\nu \geq 1}\left|V_{i}\left(G_{i, k}, \varphi_{\nu}^{(i)}\right)\right|^{2} k_{\nu}\left|\varphi_{\nu}^{(i)}(x)\right|^{2} \sum_{\nu \geq 1} \frac{1}{k_{\nu}\left(1+\eta_{i} \rho_{\nu}^{(i)}\right)^{2}} \\
& \leq \text { const } \sum_{\nu \geq 1}\left|V_{i}\left(G_{i, k}, \varphi_{\nu}^{(i)}\right)\right|^{2} k_{\nu} \sum_{\nu \geq 1} \frac{1}{k_{\nu}}=O(1) .
\end{aligned}
$$

The above holds uniformly for all $x \in \mathcal{X}_{i}$. So

$$
\sqrt{T}\left(\Omega_{i}+\Sigma_{i}\right)^{-1} V_{i}\left(G_{i}, W_{i} g_{i 0}\right)=O\left(\sqrt{T \eta_{i}}\right)=o(1)
$$

and

$$
\sqrt{T h_{i}} A_{i}^{\prime}(x)\left(\Omega_{i}+\Sigma_{i}\right)^{-1} V_{i}\left(G_{i}, W_{i} g_{i 0}\right)=O\left(\sqrt{T h_{i} \eta_{i}}\right)=o(1)
$$

By the above uniform boundedness of $A_{i, k}$, we have $\beta_{x}=0$. Similarly, by (A.18), we have

$$
W_{i} A_{i, k}(x)=\sum_{\nu \geq 1} \frac{V_{i}\left(G_{i, k}, \varphi_{\nu}^{(i)}\right)}{1+\eta_{i} \rho_{\nu}^{(i)}} \eta_{i} \rho_{\nu}^{(i)} \varphi_{\nu}^{(i)}(x) .
$$

Hence

$$
\begin{aligned}
\left|W_{i} A_{i, k}(x)\right|^{2} & \leq \sum_{\nu \geq 1}\left|V_{i}\left(G_{i, k}, \varphi_{\nu}^{(i)}\right)\right|^{2} k_{\nu}\left|\varphi_{\nu}^{(i)}(x)\right|^{2} \sum_{\nu \geq 1} \frac{\left(\eta_{i} \rho_{\nu}^{(i)}\right)^{2}}{k_{\nu}\left(1+\eta_{i} \rho_{\nu}^{(i)}\right)^{2}} \\
& \left.\leq \text { const } \sum_{\nu \geq 1}\left|V_{i}\left(G_{i, k}, \varphi_{\nu}^{(i)}\right)\right|^{2} k_{\nu} \sum_{\nu \geq 1} \frac{1}{k_{\nu}}=O_{(} 1\right),
\end{aligned}
$$

and this rate is uniform for all $x \in \mathcal{X}_{i}$. So $\alpha_{x}=0$. By definition of RKHS and $W_{i}$,

$$
\begin{aligned}
\left|W_{i} g_{i 0}(x)\right| & =\left|\left\langle W_{i} g_{i 0}, K_{x}^{(i)}\right\rangle_{\star, i}\right| \\
& =\left|\eta_{i}\left\langle g_{i 0}, K_{x}^{(i)}\right\rangle_{\mathcal{H}_{i}}\right| \\
& \leq\left\|g_{i 0}\right\|_{\mathcal{H}_{i}}\left\|K_{x}^{(i)}\right\|_{\mathcal{H}_{i}} \\
& \leq \sqrt{\eta_{i}}\left\|g_{i 0}\right\|_{\mathcal{H}_{i}} \sqrt{\left\langle K_{x}^{(i)}, K_{x}^{(i)}\right\rangle_{\star, i}} \\
& \leq \sqrt{\eta_{i}}\left\|g_{i 0}\right\|_{\mathcal{H}_{i}} O\left(h_{i}^{-1 / 2}\right) \\
& =O\left(\sqrt{\eta_{i} / h_{i}}\right) .
\end{aligned}
$$

Thus $\sqrt{T h_{i}} W_{i} g_{i 0}(x)=o(1)$.

Proof of Corollary 3.6. By (2.5),

$$
Y_{i t}-\widehat{g}_{i}\left(X_{i t}\right)-Z_{t}^{\prime} \widehat{\beta}_{i}=\epsilon_{i t}-\Delta_{i} \bar{v}_{t}+\left(g_{i 0}\left(X_{i t}\right)-\widehat{g}_{i}\left(X_{i t}\right)\right)+Z_{t}^{\prime}\left(\beta_{i 0}-\widehat{\beta}_{i}\right) .
$$

Hence $\sum_{t=1}^{T}\left\{Y_{i t}-\widehat{g}_{i}\left(X_{i t}\right)-Z_{t}^{\prime} \widehat{\beta}_{i}-\epsilon_{i t}\right\}^{2} / T \leq 4 \sum_{t=1}^{T}\left(A_{1 t}+A_{2 t}+A_{3 t}\right) / T$, where $A_{1 t}=\left\|\Delta_{i}\right\|_{2}^{2}\left\|\bar{v}_{t}\right\|_{2}^{2}$, $A_{2 t}=\left(g_{i 0}\left(X_{i t}\right)-\widehat{g}_{i}\left(X_{i t}\right)\right)^{2}, A_{3 t}=\left\|Z_{t}\right\|_{2}^{2}\left\|\widehat{\beta}_{i}-\beta_{i 0}\right\|_{2}^{2}$. By uniform boundedness of $\Delta_{i}$ and i.i.d. of 
$v_{i t}$ in Assumption A2, we have $E\left(A_{t 1}\right) \leq\left\|\Delta_{i}\right\|_{2}^{2} \operatorname{tr}\left(\Sigma_{v}\right) / N$, where $\Sigma_{v}$ is the covariance matrix of $v_{i t}$. So $\sum_{t=1}^{T} A_{1 t}=O_{P}(1 / N)=o_{P}(1)$. Also, by Lemma A.2 and Theorem 3.1,

$$
\left\|g_{i 0}-\widehat{g}_{i}\right\|_{\mathrm{sup}}=O_{P}\left(h_{i}^{-1 / 2}\left\|\theta_{i 0}-\widehat{\theta}_{i}\right\|_{i}\right)=O_{P}\left(h_{i}^{-1 / 2} r_{i, M}\right)
$$

So it follows that $\sum_{t=1}^{T} A_{2 t} / T=O_{P}\left(h_{i}^{-1} r_{i, M}^{2}\right)=o_{P}(1)$. By Proposition A.1, Lemma A.2 and Theorem 3.1, we have $A_{3 t}=O_{P}\left(T^{2 / \alpha} r_{i, M}^{2}\right)$ uniformly for all $t=1,2, \ldots, T$. So $\sum_{t=1}^{T} A_{3 t} / T=$ $O_{P}\left(T^{2 / \alpha} r_{i, M}^{2}\right)=o_{P}(1)$. Hence $\sum_{t=1}^{T}\left\{Y_{i t}-\widehat{g}_{i}\left(X_{i t}\right)-Z_{t}^{\prime} \widehat{\beta}_{i}\right\}^{2} / T-\sum_{t=1}^{T} \epsilon_{i t}^{2} / T=o_{P}(1)$. The result follows by applying Law of Large Number: $\sum_{t=1}^{T} \epsilon_{i t}^{2} / T \rightarrow \sigma_{\epsilon}^{2}$, in probability.

\section{A.3. Proofs in Section 4.2}

We prove Theorem 4.1. Let us first introduce some notation. Define

$$
\begin{array}{r}
F_{1}^{\prime}=\left(f_{11}, f_{12}, \ldots, f_{1 T}\right), F_{2}^{\prime}=\left(f_{21}, f_{22}, \ldots, f_{2 T}\right), \\
\epsilon_{i}=\left(\epsilon_{i 1}, \epsilon_{i 2}, \ldots, \epsilon_{i T}\right)^{\prime}, e_{i}=\left(e_{i 1}, e_{i 2}, \ldots, e_{i T}\right), \\
\bar{X}=\left(\bar{X}_{1}, \bar{X}_{2}, \ldots, \bar{X}_{T}\right), \bar{v}=\left(\bar{v}_{1}, \bar{v}_{2}, \ldots, \bar{v}_{T}\right), \bar{X}^{\star}=\bar{X}-\bar{v}, \\
P_{\star}=I_{T}-\Sigma_{\star}^{\prime}\left(\Sigma_{\star} \Sigma_{\star}^{\prime}\right)^{-1} \Sigma_{\star}, \widetilde{\Sigma}=\Sigma-\Sigma_{\star} .
\end{array}
$$

We can rewrite $(2.1)$ as $\left(Y_{i}-\left\langle K_{\mathbb{X}_{i}}, g_{0}\right\rangle\right)^{\prime}=\gamma_{1 i}^{\prime} F_{1}^{\prime}+\gamma_{2, i}^{\prime} F_{2}^{\prime}+\epsilon_{i}^{\prime},(2.2)$ as $\bar{X}^{*}=\bar{X}-\bar{v}=\bar{\Gamma}_{1}^{\prime} F_{1}^{\prime}+\bar{\Gamma}_{2}^{\prime} F_{2}^{\prime}$ and $(2.5)$ as $Y_{i}-\left\langle K_{\mathbb{X}_{i}}, g_{0}\right\rangle=\Sigma^{\prime} \beta_{i}+e_{i}$. Notice that we have $\Sigma=\left(F_{1}, \bar{X}^{\prime}\right)^{\prime}, \Sigma_{\star}=\left(F_{1}, \bar{X}^{* \prime}\right)^{\prime}$. By definition, we have $\Sigma P=0, \Sigma_{\star} P_{\star}=0$. Hence $F_{1}^{\prime} P=0, \bar{X} P=0, F_{1}^{\prime} P_{\star}=0, \bar{X}^{\star} P_{\star}=0, F_{2}^{\prime} P_{\star}=0$. Define $S_{M, \eta}^{\star}(g)=E\left\{S_{M, \eta}(g) \mid \mathcal{F}_{1}^{T}\right\}$. By the proof of Lemma A.1, it can be easily shown that $D S_{M, \eta}^{\star}(g)=i d$, for any $g \in \mathcal{H}$. We also have

$$
\left\|K_{x}\right\|^{2} \leq C_{\varphi}^{2} h^{-1}, \sup _{x \in \mathcal{X}}\|g(x)\| \leq C_{\varphi} h^{-1 / 2}\|g\| .
$$

The proof of Theorem 4.1 also relies on the following Lemmas A.3, A.4 and A.5. Proofs of these lemmas are provided in supplement document.

Lemma A.3. Suppose that Assumptions A2, A4 and A5 hold. Then the following holds:

$$
\begin{gathered}
E\left(\left\|P-P_{\star}\right\|_{o p}\right)=O\left(N^{-1 / 2}\right), \\
\max _{1 \leq i \leq N}\left\|E\left\{\gamma_{2 i}^{\prime} F_{2}^{\prime}\left(P-P_{\star}\right) K_{\mathbb{X}_{i}} \mid \mathcal{F}_{1}^{T}\right\}\right\|=O_{P}\left(\sqrt{\frac{T}{N h}}+\frac{T}{N \sqrt{h}}\right), \\
E\left(\left\|\widetilde{\Sigma} \widetilde{\Sigma}^{\prime}\right\|_{o p}\right)=O(T / N) .
\end{gathered}
$$

where $F_{2}=\left(f_{21}, \ldots, f_{2 T}\right)^{\prime}$. 
Lemma A.4. Suppose that Assumptions A2, A4 and A5 hold. Let $p=p(M) \geq 1$ be an $\mathcal{F}_{1}^{T}$ measurable sequence indexed by $M$ and let $\psi(\mathbb{X}, g): \mathbb{R}^{T} \times \mathcal{H} \rightarrow \mathbb{R}^{T}$ be a measurable function satisfying $\psi(\mathbb{X}, 0) \equiv 0$ and the following Lipschitz condition:

$$
\left\|\psi\left(\mathbb{X}, g_{1}\right)-\psi\left(\mathbb{X}, g_{2}\right)\right\|_{2} \leq L \sqrt{h / T}\left\|g_{1}-g_{2}\right\|_{\text {sup }}, \text { for any } g_{1}, g_{2} \in \mathcal{H},
$$

where $L>0$ is a constant. Then with $M \rightarrow \infty$,

$$
\sup _{g \in \mathcal{G}(p)}\left\|Z_{M}(g)\right\|=O_{P}\left(1+\sqrt{\log \log (N J(p, 1))}\left(J(p, 1)+N^{-1 / 2}\right)\right),
$$

where

$$
Z_{M}(g)=\frac{1}{\sqrt{N}} \sum_{i=1}^{N}\left[\psi\left(\mathbb{X}_{i}, g\right)^{\prime} P K_{\mathbb{X}_{i}}-E\left\{\psi\left(\mathbb{X}_{i}, g\right)^{\prime} P K_{\mathbb{X}_{i}} \mid \mathcal{F}_{1}^{T}\right\}\right] .
$$

Lemma A.5. Under conditions in Theorem 4.1,

$$
\left\|S_{M, \eta}\left(g_{\eta}\right)\right\|=O_{P}\left(\frac{1}{\sqrt{N T h}}+\frac{1}{N \sqrt{h}}\right)+o_{P}(\sqrt{\eta}) .
$$

Proof of Theorem 4.1. The proof consists of two parts.

Part one: Define $T_{1}(g)=g-S_{M, \eta}^{\star}\left(g+g_{0}\right)$. So

$$
T_{1}(g)=g-D S_{M, \eta}^{\star}\left(g_{0}\right) g-S_{M, \eta}^{\star}\left(g_{0}\right)=-S_{M, \eta}^{\star}\left(g_{0}\right)
$$

So we have

$$
\begin{aligned}
\left\|S_{M, \eta}^{\star}\left(g_{0}\right)\right\| & =\left\|E\left\{-\frac{1}{N T} \sum_{i=1}^{N}\left(Y_{i}-\left\langle K_{\mathbb{X}_{i}} g_{0}\right\rangle\right)^{\prime} P K_{\mathbb{X}_{i}}+W_{\eta} g_{0} \mid \mathcal{F}_{1}^{T}\right\}\right\| \\
& =\left\|E\left\{-\frac{1}{N T} \sum_{i=1}^{N} e_{i}^{\prime} P K_{\mathbb{X}_{i}}-W_{\eta} g_{0} \mid \mathcal{F}_{1}^{T}\right\}\right\| \\
& =\left\|E\left\{-\frac{1}{N T} \sum_{i=1}^{N}\left(\gamma_{1 i}^{\prime} F_{1}^{\prime}+\gamma_{2, i}^{\prime} F_{2}^{\prime}+\epsilon_{i}^{\prime}\right) P K_{\mathbb{X}_{i}}-W_{\eta} g_{0} \mid \mathcal{F}_{1}^{T}\right\}\right\| \\
& =\left\|E\left\{-\frac{1}{N T} \sum_{i=1}^{N} \gamma_{2, i}^{\prime} F_{2}^{\prime} P K_{\mathbb{X}_{i}}-W_{\eta} g_{0} \mid \mathcal{F}_{1}^{T}\right\}\right\|, \\
& =\left\|E\left\{-\frac{1}{N T} \sum_{i=1}^{N} \gamma_{2, i}^{\prime} F_{2}^{\prime}\left(P-P_{\star}\right) K_{\mathbb{X}_{i}}-W_{\eta} g_{0} \mid \mathcal{F}_{1}^{T}\right\}\right\|,
\end{aligned}
$$

where the second last equation is using independence of $\epsilon_{i}$ and $\mathbb{X}_{i}, F_{1}, F_{2}$. By directly calculations,

$$
\left\|W_{\eta} g_{0}\right\|=\sup _{\|g\|=1}\left|\left\langle W_{\eta} g_{0}, g\right\rangle\right|=\sup _{\|g\|=1}\left|\eta\left\langle g_{0}, g\right\rangle_{\mathcal{H}}\right| \leq \sup _{\|g\|=1} \sqrt{\eta}\left\|g_{0}\right\|_{\mathcal{H}} \sqrt{\eta}\|g\|_{\mathcal{H}} \leq \sqrt{\eta}\left\|g_{0}\right\|_{\mathcal{H}}
$$

For the first term, by Lemma A.3,

$$
\left\|E\left\{-\frac{1}{N T} \sum_{i=1}^{N} \gamma_{2, i}^{\prime} F_{2}^{\prime}\left(P-P_{\star}\right) K_{\mathbb{X}_{i}} \mid \mathcal{F}_{1}^{T}\right\}\right\|=O_{P}\left(\frac{1}{\sqrt{N T h}}+\frac{1}{N \sqrt{h}}\right) .
$$


As a consequence, $\left\|S_{M, \eta}^{\star}\left(g_{0}\right)\right\|=O_{P}(1 / \sqrt{N T h}+1 /(N \sqrt{h})+\sqrt{\eta})$. Hence with probability approaching one, we have

$$
\left\|T_{1}(g)\right\| \leq C\left(\frac{1}{\sqrt{N T h}}+\frac{1}{N \sqrt{h}}+\sqrt{\eta}\right) \equiv r,
$$

for some constant $C>0$. Notice that $T_{1}$ is also a contraction mapping from $\bar{B}(0, r)$ to $\bar{B}(0, r)$, so there exists a $\widetilde{g}_{1} \in \bar{B}(0, r) \subset \mathcal{H}$ such that, $T_{1}\left(\widetilde{g}_{1}\right)=\widetilde{g}_{1}$. By Taylor expansion,

$$
\widetilde{g}_{1}=T_{1}\left(\widetilde{g}_{1}\right)=\widetilde{g}_{1}-S_{M, \eta}^{\star}\left(\widetilde{g}_{1}+g_{0}\right)
$$

and hence it follows that $S_{M, \eta}^{\star}\left(\widetilde{g}_{1}+g_{0}\right)=0$. Let $g_{\eta}=\widetilde{g}_{1}+g_{0}$, we have with probability approaching one,

$$
\left\|g_{\eta}-g_{0}\right\| \leq C\left(\frac{1}{\sqrt{N T h}}+\frac{1}{N \sqrt{h}}+\sqrt{\eta}\right), \quad S_{M, \eta}^{\star}\left(g_{\eta}\right)=0 .
$$

Part two: Define $T_{2}(g)=g-S_{M, \eta}\left(g_{\eta}+g\right)$, for any $g, \Delta g \in \mathcal{H}$. Since

$$
\begin{aligned}
\Delta g & =D S_{M, \eta}^{\star}(g) \Delta g \\
& =E\left(D S_{M, \eta}(g) \Delta g \mid \mathcal{F}_{1}^{T}\right)=\frac{1}{N T} \sum_{i=1}^{N} E\left\{\tau_{i} \Delta g^{\prime} P K_{\mathbb{X}_{i}}+W_{\eta} \Delta g \mid \mathcal{F}_{1}^{T}\right\},
\end{aligned}
$$

we have that

$$
\begin{aligned}
\left\|T_{2}\left(g_{1}\right)-T_{2}\left(g_{2}\right)\right\|= & \left\|\left(g_{1}-g_{2}\right)-\left(S_{M, \eta}\left(g_{\eta}+g_{1}\right)-S_{M, \eta}\left(g_{\eta}+g_{2}\right)\right)\right\| \\
= & \left\|D S_{M, \eta}^{\star}(g)\left(g_{1}-g_{2}\right)-\left(S_{M, \eta}\left(g_{\eta}+g_{1}\right)-S_{M, \eta}\left(g_{\eta}+g_{2}\right)\right)\right\| \\
= & \| \frac{1}{N T} \sum_{i=1}^{N}\left\{\tau_{i}\left(g_{1}-g_{2}\right)^{\prime} P K_{\mathbb{X}_{i}}+W_{\eta}\left(g_{1}-g_{2}\right)\right. \\
& \left.-E\left(\tau_{i}\left(g_{1}-g_{2}\right)^{\prime} P K_{\mathbb{X}_{i}}+W_{\eta}\left(g_{1}-g_{2}\right) \mid \mathcal{F}_{1}^{T}\right)\right\} \| \\
= & \left\|\frac{1}{N T} \sum_{i=1}^{N}\left\{\tau_{i}\left(g_{1}-g_{2}\right)^{\prime} P K_{\mathbb{X}_{i}}-E\left(\tau_{i}\left(g_{1}-g_{2}\right)^{\prime} P K_{\mathbb{X}_{i}} \mid \mathcal{F}_{1}^{T}\right)\right\}\right\| \\
\equiv & \left\|\kappa\left(g_{1}-g_{2}\right)\right\|,
\end{aligned}
$$

where $\kappa(g)=\sum_{i=1}^{N}\left(\tau_{i} g^{\prime} P K_{\mathbb{X}_{i}}-E\left\{\tau_{i} g^{\prime} P K_{\mathbb{X}_{i}} \mid \mathcal{F}_{1}^{T}\right\}\right) /(N T)$. Let $\Psi\left(\mathbb{X}_{i}, g\right)=\sqrt{h} \tau_{i} g / T$. It follows that, for any $g_{1}, g_{2} \in \mathcal{H}$,

$$
\begin{aligned}
\left\|\Psi\left(\mathbb{X}_{i}, g_{1}\right)-\Psi\left(\mathbb{X}_{i}, g_{1}\right)\right\|_{2} & \leq \frac{\sqrt{h}}{T}\left\|\tau_{i}\left(g_{1}-g_{2}\right)\right\|_{2} \\
& \leq \frac{\sqrt{h}}{T} \sqrt{\sum_{t=1}^{T}\left|g_{1}\left(X_{i t}\right)-g_{2}\left(X_{i t}\right)\right|^{2}} \leq \sqrt{\frac{h}{T}}\left\|g_{1}-g_{2}\right\|_{\text {sup }} .
\end{aligned}
$$

Let $\widetilde{g} \equiv\left(g_{1}-g_{2}\right) /\left(c_{\varphi} h^{-1 / 2}\left\|g_{1}-g_{2}\right\|\right)$, then

$$
\|\widetilde{g}\|_{\text {sup }}=\frac{\left\|g_{1}-g_{2}\right\|_{\text {sup }}}{c_{\varphi} h^{-1 / 2}\left\|g_{1}-g_{2}\right\|} \leq 1
$$


and

$$
\left\|\widetilde{g}_{\eta}\right\|_{\mathcal{H}}=\frac{\left\|g_{1}-g_{2}\right\|_{\mathcal{H}}}{c_{\varphi} h^{-1 / 2}\left\|g_{1}-g_{2}\right\|} \leq \frac{1}{c_{\varphi} \sqrt{h^{-1} \eta}} .
$$

So $\widetilde{g} \in \mathcal{G}(p)$, with $p=1 /\left(c_{\varphi} \sqrt{h^{-1} \eta}\right)$. By Lemma A.4,

$$
\frac{\left\|Z_{M}\left(g_{1}-g_{2}\right)\right\|}{c_{\varphi} h^{-1 / 2}\left\|g_{1}-g_{2}\right\|}=\left\|Z_{M}(\widetilde{g})\right\|=O_{P}\left(1+\sqrt{\log \log (N J(p, 1))}\left(J(p, 1)+N^{-1 / 2}\right)\right)=O_{P}\left(b_{N, p}\right) .
$$

So by assumption $b_{N, p}=o_{P}(\sqrt{N} h)$, we have

$$
\begin{aligned}
\left\|\kappa\left(g_{1}-g_{2}\right)\right\| & =\left\|\frac{1}{\sqrt{N h}} Z_{M}\left(g_{1}-g_{2}\right)\right\| \\
& =c_{\varphi} \frac{1}{\sqrt{N h}}\left\|g_{1}-g_{2}\right\| O_{P}\left(b_{N, p}\right)=O_{P}\left(\frac{b_{N, p}}{\sqrt{N h}}\right)\left\|g_{1}-g_{2}\right\|=o_{P}(1)\left\|g_{1}-g_{2}\right\|,
\end{aligned}
$$

where the terms $O_{P}, o_{P}$ do not depend on $g_{1}, g_{2}$. Hence with probability approaching one, uniformly for any $g_{1}, g_{2},\left\|T_{2}\left(g_{1}\right)-T_{2}\left(g_{2}\right)\right\| \leq \frac{1}{2}\left\|g_{1}-g_{2}\right\|$. Also with probability approaching one, uniformly for $g$,

$$
\left\|T_{2}(g)\right\| \leq\left\|T_{2}(g)-T_{2}(0)\right\|+\left\|T_{2}(0)\right\| \leq \frac{1}{2}\|g\|+\left\|S_{M, \eta}\left(g_{\eta}\right)\right\| .
$$

By Lemma A.5, with probability approaching one,

$$
\left\|S_{M, \eta}\left(g_{\eta}\right)\right\| \leq C\left(\frac{1}{\sqrt{N T h}}+\frac{1}{N \sqrt{h}}+\sqrt{\eta}\right) \equiv R / 2
$$

for some $C>0$. Hence with probability approaching one, it follows that $\sup _{\|g\| \leq R}\left\|T_{2}(g)\right\| \leq$ $R / 2+R / 2=R$. The above implies that $T_{2}$ is a contraction mapping from $\bar{B}(0, R)$ to itself. By contraction mapping theorem, there exists $\widetilde{g}_{2} \in \bar{B}(0, R)$ such that $\widetilde{g}_{2}=T_{2}\left(\widetilde{g}_{2}\right)=\widetilde{g}_{2}-S_{M, \eta}\left(g_{\eta}+\widetilde{g}_{2}\right)$. Hence $S_{M, \eta}\left(g_{\eta}+\widetilde{g}_{2}\right)=0$. So $\widehat{g}=g_{\eta}+\widetilde{g}_{2}$. Therefore,

$$
\left\|\widehat{g}-g_{0}\right\| \leq\left\|g_{\eta}-g_{0}\right\|+\left\|\widetilde{g}_{2}\right\|=O_{P}\left(\frac{1}{\sqrt{N T h}}+\frac{1}{N \sqrt{h}}+\sqrt{\eta}\right) .
$$

\section{A.4. Proofs in Section 4.3}

We will prove Theorems 4.2 and 4.3, and Proposition 4.5. Let us introduce some additional notation and preliminaries. Let $m$ be the increasing sequence of integers provided in Theorems 4.2 and 4.3. For any fixed $x_{0} \in \mathcal{X}$, define $V_{N T}=\frac{1}{N T} \sum_{i=1}^{N} K_{\mathbb{X}_{i}}\left(x_{0}\right)^{\prime} P K_{\mathbb{X}_{i}}\left(x_{0}\right), A_{N T}=V_{N T}^{-1 / 2}$. Let $V_{N T m}=\sum_{i=1}^{N} \phi_{m}^{\prime} \Phi_{i}^{\prime} P \Phi_{i} \phi_{m} /(N T), A_{N T m}=V_{N T m}^{-1 / 2}$ and $H_{N T m}=\sum_{i=1}^{N} \Phi_{i}^{\prime} P \Phi_{i} /(N T)$, where

$$
\Phi_{i}=\left(\begin{array}{cccc}
\varphi_{1}\left(X_{i 1}\right) & \varphi_{2}\left(X_{i 1}\right) & \cdots & \varphi_{m}\left(X_{i 1}\right) \\
\varphi_{1}\left(X_{i 2}\right) & \varphi_{2}\left(X_{i 2}\right) & \cdots & \varphi_{m}\left(X_{i 2}\right) \\
& & \cdots & \\
\varphi_{1}\left(X_{i T}\right) & \varphi_{2}\left(X_{i T}\right) & \cdots & \varphi_{m}\left(X_{i T}\right)
\end{array}\right), \quad \phi_{m}=\left(\frac{\varphi_{1}\left(x_{0}\right)}{1+\eta \rho_{1}}, \frac{\varphi_{2}\left(x_{0}\right)}{1+\eta \rho_{2}}, \cdots, \frac{\varphi_{m}\left(x_{0}\right)}{1+\eta \rho_{m}}\right)^{\prime} .
$$


The proof of Theorems 4.2 and 4.3 rely on the following Lemmas A.6, A.7 and A.8. Proofs of these lemmas can be found in supplement document.

Lemma A.6. Under Assumptions A2, A4 and A5, suppose $m=o(\sqrt{N})$, then $\left\|H_{N T m}-I_{m}\right\|_{F}=$ $o_{P}(1), \lambda_{\min }^{-1}\left(H_{N T m}\right)=O_{P}(1)$ and $\lambda_{\max }\left(H_{N T m}\right)=O_{P}(1)$.

Lemma A.7. Under Assumptions A2, A4 and A5, suppose $h^{-1}=o_{P}(\sqrt{N}), D_{m}=o_{P}(1)$, then for any $x_{0} \in \mathcal{X}, A_{N T}=O_{P}(1)$.

Lemma A.8. Under Assumptions A2, A4 and A5, suppose $D_{m}=o_{P}(\sqrt{N}), m=o_{P}(\sqrt{N})$, for ant $x_{0} \in \mathcal{X}$, we have

$$
\sqrt{N T} A_{N T m}\left(\frac{1}{N T} \sum_{i=1}^{N} \phi_{m}^{\prime} \Phi_{i}^{\prime} P \epsilon_{i}\right) \stackrel{d}{\rightarrow} N\left(0, \sigma_{\epsilon}^{2}\right) .
$$

Proof of Theorem 4.2. Let $g=\widehat{g}-g_{0}$. By the fact $D S_{M, \eta}^{\star}\left(g_{0}\right)=i d$ (see Section A.3) and $S_{M, \eta}(g+$ $\left.g_{0}\right)=0$, we have

$$
\begin{aligned}
\left\|g+S_{M, \eta}\left(g_{0}\right)\right\| & =\left\|D S_{M, \eta}^{\star}\left(g_{0}\right) g+S_{M, \eta}\left(g_{0}\right)\right\| \\
& =\left\|S_{M, \eta}^{\star}\left(g+g_{0}\right)-S_{M, \eta}^{\star}\left(g_{0}\right)-S_{M, \eta}\left(g+g_{0}\right)+S_{M, \eta}\left(g_{0}\right)\right\| \\
& =\left\|\frac{1}{N T} \sum_{i=1}^{N}\left(\tau_{i} g^{\prime} P K_{\mathbb{X}_{i}}-E\left(\tau_{i} g^{\prime} P K_{\mathbb{X}_{i}}\right)\right)+W_{\eta} g-E\left(W_{\eta} g \mid \mathcal{F}_{1}^{T}\right)\right\| \\
& \leq\|\kappa(g)\|,
\end{aligned}
$$

where $\kappa(g)$ is defined in Part two of the proof of Theorem 4.1. By (A.24) with $g_{1}-g_{2}$ therein replaced by $g$ we have $\|\kappa(g)\|=O_{P}\left(\frac{b_{N, p}}{\sqrt{N} h}\right)\|g\|$. Hence

$$
\left\|g+S_{M, \eta}\left(g_{0}\right)\right\|=O_{P}\left(\frac{b_{N, p}}{\sqrt{N h}}\right)\|g\|=O_{P}\left(\frac{b_{N, p}}{\sqrt{N h}}\left(\frac{1}{\sqrt{N T h}}+\frac{1}{N \sqrt{h}}+\sqrt{\eta}\right)\right) .
$$

For fixed $x_{0} \in \mathcal{X}$

$$
\begin{aligned}
A_{N T}\left|g\left(x_{0}\right)+S_{M, \eta}\left(g_{0}\right)\left(x_{0}\right)\right| & \leq A_{N T}\left\|g+S_{M, \eta}\left(g_{0}\right)\right\|_{\text {sup }} \\
& \leq c_{\varphi} h^{-1 / 2}\left\|g+S_{M, \eta}\left(g_{0}\right)\right\| \\
& =O_{P}\left(\frac{b_{N, p}}{\sqrt{N} h}\left(\frac{1}{\sqrt{N T} h}+\frac{1}{N h}+\sqrt{\frac{\eta}{h}}\right)\right) .
\end{aligned}
$$

Since $g_{0} \in \mathcal{H}$ is fixed,

$$
\left\|W_{\eta} g_{0}\right\|=\sup _{\|\widetilde{g}\|=1}\left\langle W_{\eta} g_{0}, \widetilde{g}\right\rangle=\sup _{\|\widetilde{g}\|=1} \eta\left\langle g_{0}, \widetilde{g}\right\rangle_{\mathcal{H}} \leq \sup _{\|\widetilde{g}\|=1} \eta\left\|g_{0}\right\|_{\mathcal{H}}\|\widetilde{g}\|_{\mathcal{H}} \leq \sqrt{\eta}\left\|g_{0}\right\|_{\mathcal{H}}
$$

It follows from $(\mathrm{A} .25)$ that $\left\|W_{\eta} g_{0}\right\| \leq \sqrt{\eta}\left\|g_{0}\right\|_{\mathcal{H}}=O_{P}(\sqrt{\eta})$, and hence

$$
\left|W_{\eta} g_{0}\left(x_{0}\right)\right| \leq c_{\varphi} h^{-1 / 2}\left\|W_{\eta} g_{0}\right\|=O_{P}(\sqrt{\eta / h}) .
$$


Hence, we have

$$
A_{N T}\left|g\left(x_{0}\right)+S_{M, \eta}\left(g_{0}\right)\left(x_{0}\right)-W_{\eta} g_{0}\left(x_{0}\right)\right|=O_{P}\left(\frac{b_{N, p}}{\sqrt{N h}}\left(\frac{1}{\sqrt{N T} h}+\frac{1}{N h}+\sqrt{\frac{\eta}{h}}\right)\right) .
$$

Proof of Theorem 4.3. By Theorem 4.2, the asymptotic distribution of $\sqrt{N T} A_{N T}\left(\widehat{g}\left(x_{0}\right)-g_{0}\left(x_{0}\right)+\right.$ $\left.W_{\eta} g_{0}\right)$ is the same as $\sqrt{N T} A_{N T}\left[-S_{M, \eta}\left(g_{0}\right)\left(x_{0}\right)+W_{\eta} g_{0}\left(x_{0}\right)\right]=\sqrt{N T} A_{N T}\left[\frac{1}{N T} \sum_{i=1}^{N}\left(Y_{i}-\tau_{i} g_{0}\right)^{\prime} P K_{\mathbb{X}_{i}}\left(x_{0}\right)\right]$.

By $Y_{i}=\tau_{i} g_{0}+\Sigma^{\prime} \beta_{i}+e_{i}(4.2), \Sigma P=0$ (see Section A.3) and $e_{i}=\epsilon_{i}-\bar{v}^{\prime} \Delta_{i}^{\prime}$, it yields that

$$
\begin{aligned}
& A_{N T}\left[\frac{1}{N T} \sum_{i=1}^{N}\left(Y_{i}-\tau_{i} g_{0}\right)^{\prime} P K_{\mathbb{X}_{i}}\left(x_{0}\right)\right] \\
= & A_{N T}\left(\frac{1}{N T} \sum_{i=1}^{N} K_{\mathbb{X}_{i}}^{\prime}\left(x_{0}\right) P \epsilon_{i}\right)-A_{N T}\left(\frac{1}{N T} \sum_{i=1}^{N} K_{\mathbb{X}_{i}}^{\prime}\left(x_{0}\right) P \bar{v}^{\prime} \Delta_{i}^{\prime}\right) \\
\equiv & A_{N T} \zeta-A_{N T} \xi .
\end{aligned}
$$

Let $\zeta_{m}=\sum_{i=1}^{N} \phi_{m}^{\prime} \Phi_{i}^{\prime} P \epsilon_{i} /(N T)$ and $\xi_{m}=\sum_{i=1}^{N} \phi_{m}^{\prime} \Phi_{i}^{\prime} P \bar{v}^{\prime} \Delta_{i}^{\prime} /(N T)$. To prove the result of the theorem, it is sufficient to prove the following:

$$
\begin{aligned}
A_{N T}-A_{N T m} & =o_{P}(1), \\
A_{N T} & =O_{P}(1), \\
\sqrt{N T} A_{N T m} \xi_{m} & =o_{P}(1), \\
\sqrt{N T}\left(\xi-\xi_{m}\right) & =o_{P}(1), \\
\sqrt{N T}\left(\zeta-\zeta_{m}\right) & =o_{P}(1), \\
\sqrt{N T} A_{N T m} \zeta_{m} & \stackrel{d}{\rightarrow} N(0,1) .
\end{aligned}
$$

(A.26) and (A.27) are guaranteed by Lemma A.7; (A.31) follows from Lemma A.8. For (A.28), by the expression of $A_{N T m}$ and Lemma A.6, we get,

$$
\begin{aligned}
\left|A_{N T m} \xi_{m}\right| & =\left|A_{N T m} \phi_{m}^{\prime} \frac{1}{N T} \sum_{i=1}^{N} \Phi_{i}^{\prime} P \bar{v}^{\prime} \Delta_{i}^{\prime}\right| \\
& \leq\left\|A_{N T m} \phi_{m}\right\|_{2} \times\left\|\frac{1}{N T} \sum_{i=1}^{N} \Phi_{i}^{\prime} P \bar{v}^{\prime} \Delta_{i}^{\prime}\right\|_{2} \\
& \leq \sqrt{\frac{\left\|\phi_{m}\right\|_{2}^{2}}{\phi_{m}^{\prime} H_{N T m} \phi_{m}}}\left\|\frac{1}{N T} \sum_{i=1}^{N} \Phi_{i}^{\prime} P \bar{v}^{\prime} \Delta_{i}^{\prime}\right\|_{2} \\
& \leq \lambda_{\min }^{-1 / 2}\left(H_{N T m}\right)\left\|\frac{1}{N T} \sum_{i=1}^{N} \Phi_{i}^{\prime} P \bar{v}^{\prime} \Delta_{i}^{\prime}\right\|_{2} .
\end{aligned}
$$

Recall $\Delta_{i}=\gamma_{2 i}^{\prime}\left(\bar{\Gamma}_{2} \bar{\Gamma}_{2}^{\prime}\right)^{-1} \bar{\Gamma}_{2} \equiv \gamma_{2 i}^{\prime} \widetilde{M}$ (see Assumption A2(d)), which leads to

$$
\left\|\sum_{i=1}^{N} \Phi_{i}^{\prime} P \bar{v}^{\prime} \Delta_{i}^{\prime}\right\|_{2}^{2}=\operatorname{Tr}\left\{\left(\sum_{i=1}^{N} \Phi_{i}^{\prime} P \bar{v}^{\prime} \Delta_{i}^{\prime}\right)\left(\sum_{i=1}^{N} \Delta_{i} \bar{v} P \Phi_{i}\right)\right\} .
$$


In matrix form, the above becomes

$$
\begin{aligned}
& \operatorname{Tr}\left\{\left(\Phi_{1}^{\prime}, \Phi_{2}^{\prime}, \ldots, \Phi_{N}^{\prime}\right) P_{N} \bar{v}_{N}^{\prime} \widetilde{M}_{N}^{\prime} \gamma_{2} \gamma_{2}{ }^{\prime} \widetilde{M}_{N} \bar{v}_{N} P_{N}\left(\Phi_{1}^{\prime}, \Phi_{2}^{\prime}, \ldots, \Phi_{N}^{\prime}\right)^{\prime}\right\} \\
\leq & \lambda_{\max }\left(\gamma_{2} \gamma_{2}^{\prime}\right) \lambda_{\max }\left(\widetilde{M}_{N}^{\prime} \widetilde{M}_{N}\right) \lambda_{\max }\left(\bar{v}_{N}^{\prime} \bar{v}_{N}\right) \operatorname{Tr}\left\{\sum_{i=1}^{N} \Phi_{i}^{\prime} P \Phi_{i}\right\},
\end{aligned}
$$

where $P_{N}$ is defined in Section 4.1, $\widetilde{M}_{N}=I_{N} \otimes \widetilde{M}, \bar{v}_{N}=I_{N} \otimes \bar{v}$.

By Lemma A.3, Assumption A2 and Lemma A.6 ,we have

$$
\lambda_{\max }\left(\bar{v}_{N}^{\prime} \bar{v}_{N}\right)=\left\|\widetilde{\Sigma} \widetilde{\Sigma}^{\prime}\right\|_{\text {op }}=O_{P}(T / N), \quad \lambda_{\max }\left(\widetilde{M}_{N}^{\prime} \widetilde{M}_{N}\right)=\lambda_{\max }\left(\left(\bar{\Gamma}_{2} \bar{\Gamma}_{2}^{\prime}\right)^{-1}\right)=O_{P}(1),
$$

and

$$
\operatorname{Tr}\left\{\sum_{i=1}^{N} \Phi_{i}^{\prime} P \Phi_{i}\right\}=N \operatorname{TTr}\left(H_{N T m}\right)=O_{P}(N T m) .
$$

So it can be seen that,

$$
\left|A_{N T m} \xi_{m}\right|=O_{P}\left(\frac{\sqrt{\lambda_{\max }\left(\gamma_{2} \gamma_{2}^{\prime}\right) m}}{N}\right)
$$

thus

$$
\sqrt{N T} A_{N T m} \xi_{m}=O_{P}\left(\sqrt{\frac{\lambda_{\max }\left(\gamma_{2} \gamma_{2}^{\prime}\right) m T}{N}}\right)=o_{P}(1)
$$

and hence (A.28) is true.

Similar to (S.20) in the proof of Lemma A.5 (see supplement document), it can be shown that

$$
\left|\xi-\xi_{m}\right|=O_{P}\left(\frac{D_{m}}{\sqrt{N T h}}+\frac{D_{m}}{N \sqrt{h}}\right) .
$$

More explicitly, the proof of (A.32) follows by replacing $K_{\mathbb{X}_{i}}$ in the expression of $T_{2}$ with $\Phi_{i} \phi_{m}$, and by a line-by-line check. Therefore, we have

$$
\sqrt{N T}\left|\xi-\xi_{m}\right|=O_{P}\left(\frac{D_{m}}{\sqrt{h}}+\frac{D_{m} \sqrt{T}}{\sqrt{N h}}\right)=o_{P}(1),
$$

i.e., (A.29) holds.

Let $\mathcal{D}_{1}^{T}=\sigma\left(f_{1 t}, f_{2 t}, X_{i t}: t \in[T], i \in[N]\right)$. By independence of $\epsilon_{i}$ and $\mathcal{D}_{1}^{T}$, we have $E(\mid \zeta-$ $\left.\left.\zeta_{m}\right|^{2} \mid \mathcal{D}_{1}^{T}\right)=\sum_{i=1}^{N} R_{i}^{\prime} P R_{i} /(N T)$, where $R_{i}=\left(K_{\mathbb{X}_{i}}\left(x_{0}\right)-\Psi_{i} \phi_{m}\right)^{\prime} P \epsilon_{i}$. Let $R_{x_{0}}(\cdot)=\sum_{\nu \geq m+1} \frac{\varphi_{\nu}\left(x_{0}\right) \varphi_{\nu}(\cdot)}{1+\eta \rho_{\nu}}$, since $\mathcal{F}_{1}^{T} \subset \mathcal{D}_{1}^{T}$, it follows that,

$$
\begin{aligned}
E\left(\left|\zeta-\zeta_{m}\right|^{2} \mid \mathcal{F}_{1}^{T}\right) & =E\left(\frac{1}{N^{2} T^{2}} \sum_{i=1}^{N} R_{i}^{\prime} P R_{i} \mid \mathcal{F}_{1}^{T}\right) \\
& =\frac{1}{N T} V\left(R_{x_{0}}, R_{x_{0}}\right) \\
& =\frac{1}{N T} \sum_{\nu=m+1}^{\infty} \frac{\varphi_{\nu}^{2}\left(x_{0}\right)}{\left(1+\eta \rho_{\nu}\right)^{2}} \\
& \leq \frac{1}{N T} c_{\varphi}^{2} D_{m} \\
& =O_{P}\left(\frac{D_{m}}{N T}\right)
\end{aligned}
$$


so $\zeta-\zeta_{m}=O_{P}\left(\sqrt{D_{m} /(N T)}\right)$ which implies that (A.30) is valid. Proof completed.

Proof of Proposition 4.5. By $Y_{i}=\tau_{i} g_{0}+\Sigma^{\prime} \beta_{i}+e_{i}, \Sigma P=0, e_{i}=\epsilon_{i}-\bar{v}^{\prime} \Delta_{i}^{\prime}$, we have

$$
\begin{aligned}
& \frac{1}{N T} \sum_{i=1}^{N}\left(Y_{i}-\tau_{i} \widehat{g}\right)^{\prime} P\left(Y_{i}-\tau_{i} \widehat{g}\right) \\
= & \frac{1}{N T} \sum_{i=1}^{N}\left(\tau_{i}\left(g_{0}-\widehat{g}\right)\right)^{\prime} P\left(\tau_{i}\left(g_{0}-\widehat{g}\right)\right)+\frac{2}{N T} \sum_{i=1}^{N}\left(\tau_{i}\left(g_{0}-\widehat{g}\right)\right)^{\prime} P e_{i}+\frac{1}{N T} \sum_{i=1}^{N} e_{i}^{\prime} P e_{i} \\
\equiv & T_{1}+T_{2}+T_{3} .
\end{aligned}
$$

By Theorem 4.1,

$$
\left\|\widehat{g}-g_{0}\right\|_{\sup } \leq c_{\varphi} h^{-1 / 2}\left\|\widehat{g}-g_{0}\right\|=O_{P}\left(\frac{1}{\sqrt{N T} h}+\frac{1}{N h}+\sqrt{\frac{\eta}{h}}\right)=o_{P}(1) .
$$

So $\left|T_{1}\right| \leq \sum_{i=1}^{N} \sum_{t=1}^{T}\left|g_{0}\left(X_{i t}-\widehat{g}\left(X_{i t}\right)\right)\right|^{2} /(N T) \leq\left\|\widehat{g}-g_{0}\right\|_{\text {sup }}^{2}=o_{P}(1)$.

By the definitions of $\bar{v}$ and $\widetilde{\Sigma}$ and by Lemma A.3, we have $E\left(\left\|\bar{v} \bar{v}^{\prime}\right\|_{\text {op }}\right)=E\left(\|\widetilde{\Sigma} \widetilde{\Sigma}\|_{\text {op }}\right)=$ $O(T / N))$. Hence it holds that

$$
\begin{aligned}
E\left(\left\|e_{i}\right\|_{2}^{2}\right) & =E\left(\epsilon_{i}^{\prime} \epsilon_{i}\right)+E\left(\Delta_{i}^{\prime} \bar{v} \bar{v}^{\prime} \Delta_{i}\right)-2 E\left(\epsilon_{i}^{\prime} \bar{v}^{\prime} \Delta_{i}\right) \\
& \leq T \sigma_{\epsilon}^{2}+E\left(\left\|\bar{v} \bar{v}^{\prime}\right\|_{\text {op }}\right) \sup _{1 \leq i \leq N}\left\|\Delta_{i}\right\|_{2}^{2}=O(T) .
\end{aligned}
$$

It then follows from Cauchy inequality that

$$
\begin{aligned}
\left|T_{2}\right| & \leq \frac{2}{N T} \sum_{i=1}^{N}\left\|\tau_{i}\left(g_{0}-\widehat{g}\right)\right\|_{2}\left\|e_{i}\right\|_{2} \\
& \leq \frac{2}{N T} \sum_{i=1}^{N}\left\|e_{i}\right\|_{2} \sqrt{T\left\|\widehat{g}-g_{0}\right\|_{\text {sup }}} \\
& =O_{P}(1) \sqrt{\left\|\widehat{g}-g_{0}\right\|_{\text {sup }}}=o_{P}(1) .
\end{aligned}
$$

Meanwhile, the following decomposition holds

$$
\begin{aligned}
T_{3} & =\frac{1}{N T} \sum_{i=1}^{N} \epsilon_{i}^{\prime} P \epsilon_{i}+\frac{1}{N T} \sum_{i=1}^{N} \Delta_{i}^{\prime} \bar{v} P \bar{v}^{\prime} \Delta_{i}-\frac{2}{N T} \sum_{i=1}^{N} \Delta_{i}^{\prime} \bar{v} P \epsilon_{i} \\
& =\frac{1}{N T} \sum_{i=1}^{N} \epsilon_{i}^{\prime} P_{\star} \epsilon_{i}+\frac{1}{N T} \sum_{i=1}^{N} \epsilon_{i}^{\prime}\left(P-P_{\star}\right) \epsilon_{i}+\frac{1}{N T} \sum_{i=1}^{N} \Delta_{i}^{\prime} \bar{v} P \bar{v}^{\prime} \Delta_{i}-\frac{2}{N T} \sum_{i=1}^{N} \Delta_{i}^{\prime} \bar{v} P \epsilon_{i} \\
& \equiv T_{31}+T_{32}+T_{33}-T_{34} .
\end{aligned}
$$

We handle the above terms $T_{31}, T_{32}, T_{33}, T_{34}$ respectively. By Lemma A.3, it follows that

$$
\left|T_{32}\right| \leq \frac{1}{N T}\left\|P-P_{\star}\right\|_{\text {op }} \sum_{i=1}^{N} \epsilon_{i}^{\prime} \epsilon_{i}=O_{P}\left(N^{-1 / 2}\right)=o_{P}(1) .
$$


In the meantime,

$$
\left|T_{33}\right| \leq \frac{1}{N T} \sup _{1 \leq i \leq N}\left\|\Delta_{i}\right\|_{2}^{2} \sum_{i=1}^{N}\left\|\bar{v} \bar{v}^{\prime}\right\|_{\mathrm{op}}=O_{P}\left(\frac{1}{N}\right)=o_{P}(1),
$$

and

$$
\left|T_{34}\right| \leq \frac{2}{N T}\left\|\epsilon_{i}\right\|_{2}\left\|\bar{v}^{\prime} \Delta_{i}\right\| \leq 2 \sqrt{\frac{\sum_{i=1}^{N} \epsilon_{i}^{\prime} \epsilon}{N T}} \sqrt{\frac{\sum_{i=1}^{N} \Delta_{i}^{\prime} \bar{v} \bar{v}^{\prime} \Delta_{i}}{N T}}=O_{P}\left(\frac{1}{\sqrt{N}}\right)=o_{P}(1) .
$$

Next we look at $T_{31}$. By direct examinations,

$$
E\left(T_{31} \mid \mathcal{F}_{1}^{T}\right)=\frac{1}{T} \operatorname{Tr}\left\{P_{\star} E\left(\epsilon_{i} \epsilon_{i}^{\prime} \mid \mathcal{F}_{1}^{T}\right)\right\}=\sigma_{\epsilon}^{2} \frac{1}{T} \operatorname{Tr}\left(P_{\star}\right)=\sigma_{\epsilon}^{2} \frac{T-\left(q_{1}+d\right)}{T},
$$

and by Chebyshev's inequality, for any $\epsilon>0$,

$$
\begin{aligned}
P\left(\left|T_{31}-E\left(T_{31} \mid \mathcal{F}_{1}^{T}\right)\right|>\epsilon \mid \mathcal{F}_{1}^{T}\right) & \leq \frac{1}{N} \frac{E\left\{\left|\epsilon_{1}^{\prime} P_{\star} \epsilon_{1} / T-E\left(T_{31} \mid \mathcal{F}_{1}^{T}\right)\right|^{2}\right\} \mid \mathcal{F}_{1}^{T}}{\epsilon^{2}} \\
& \leq \frac{1}{N \epsilon^{2}} E\left(\left|\epsilon_{1}^{\prime} P_{\star} \epsilon_{1} / T\right|^{2} \mid \mathcal{F}_{1}^{T}\right) \\
& \leq \frac{1}{N T^{2} \epsilon^{2}} E\left(\left|\epsilon_{1}^{\prime} \epsilon_{1}\right|^{2} \mid \mathcal{F}_{1}^{T}\right) \\
& \leq \frac{1}{N T^{2} \epsilon^{2}}\left(T E\left(\epsilon_{11}^{4}\right)+T^{2} \sigma_{\epsilon}^{4}\right) \\
& =o(1) .
\end{aligned}
$$

So $T_{31} \rightarrow \sigma_{\epsilon}^{2}\left(T-q_{1}-d\right) / T$ in probability. Since $T_{32}, T_{33}, T_{34}$ are all $o_{P}(1)$ as shown in the above, we have $T_{3} \rightarrow \sigma_{\epsilon}^{2}\left(T-q_{1}-d\right) / T$ in probability. Proof completed.

\section{References}

Bai, J. (2009). Panel data models with interactive fixed effects. Econometrica, 77(4):1229-1279.

Bai, J. and Ng, S. (2006). Confidence intervals for diffusion index forecasts and inference for factoraugmented regressions. Econometrica, 74(4):1133-1150.

Berlinet, A. and Thomas-Agnan, C. (2004). Reproducing Kernel Hilbert Spaces in Probability and Statistics. Kluwer Academic, Boston, MA.

Bradley, R. C. (2005). Basic properties of strong mixing conditions. a survey and some open questions. Probability Surveys, 2(2):107-144.

Braun, M. (2006). Accurate error bounds for the eigenvalues of the kernel matrix. Journal of Machine Learning Research, 7:2303-2328.

Cai, Z., Fang, Y., and Xu, q. (2016). Inferences for varying-coefficient panel data models with cross-sectional dependence. Working Paper.

Carneiro, P., Hansen, K., and Heckman, J. (2003). Estimating distributions of treatment effects with an application to the returns to schooling and measurement of the effects of uncertainty on college choice. International Economic Review, 44(2):361-422. 
Chen, X. (2007). Large sample sieve estimation of semi-nonparametric models. Handbook of Econometrics, 6:5549-5632.

Cheng, G. and Shang, Z. (2015). Joint asymptotics for semi-nonparametric regression models with partially linear structure. The Annals of Statistics, 43(3):1351-1390.

Craven, P. and Wahba, G. (1978). Smoothing noisy data with spline functions. Numerische Mathematik, 31(4):377-403.

Cucker, F. and Smale, S. (2001). On the mathematical foundations of learning. American Mathematical Society, 39(1):1-49.

Cunha, F., Heckman, J., and Navarro, S. (2005). Separating uncertainty from heterogeneity in life cycle earnings. Oxford Economic papers, 57(2):191-261.

Dehling, H. (1983). Limit theorems for sums of weakly dependent banach space valued random variables. Probability Fields and Related Fields, 63(3):393-432.

Dudley, R. M., Hahn, M. G., and Kuelbs, J. (1992). Probability in Banach space. Springer Science Business Media.

Eberhardt, M., Helmers, C., and Strauss, H. (2013). Do spillovers matter when estimating private returns to R\&D? Review of Economics and Statistics, 95(2):436-448.

Fan, J. and Yao, Q. (2003). Nonlinear Time Series: Nonparametric and Parametric Methods. Springer Science Business Media, LLC.

Freyberger, J. (2012). Nonparametric panel data models with interactive fixed effects. Working Paper.

Gu, C. (2013). Smoothing spline ANOVA models, vol 297. Springer Science Business Media, LLC.

Gu, C. and Qiu, C. (1993). Smoothing spline density estimation: Theory. The Annals of Statistics, pages $217-234$.

Hashiguchi, Y. (2015). Allocation efficiency in china: an extension of the dynamic olley-pakes productivity decomposition. Working Paper.

Hofmann, T., Schlkopf, B., and Smola, A. J. (2008). Kernel methods in machine learning. The Annals of Statistics, pages 1171-1220.

Holly, S., Pesaran, M. H., and Yamagata, T. (2010). A spatio-temporal model of house prices in the usa. Journal of Econometrics, 158(1):160-173.

Huang, X. (2013). Nonparametric estimation in large panels with cross-sectional dependence. Econometric Reviews, 32(5-6):754-777.

Jin, S. and Su, L. (2013). A nonparametric poolability test for panel data models with cross section dependence. Econometric Reviews, 32(4):469-512.

Kosorok, M. (2008). Introduction to empirical processes and semiparametric inference. Springer: New York.

Lu, J., Cheng, G., and Liu, H. (2016). Nonparametric heterogeneity testing for massive data. arXiv preprint arXiv:1601.

Ludvigson, S. C. and Ng, S. (2009). Macro factors in bond risk premia. Review of Financial Studies, 22(12):5027-5067.

Ludvigson, S. C. and Ng, S. (2016). A factor analysis of bond risk premia. Handbook of Empirical Economics and Finance, pages 313-372.

Mammen, E. and van de Geer, S. (1997). Penalized quasi-likelihood estimation in partial linear models. The Annals of Statistics, pages 1014-1035.

Melitz, M. J. (2003). The impact of trade on intra-industry reallocations and aggregate industry productivity. Econometrica, 71(6):1695-1725. 
Moon, H. R. and Weidner, M. (2010). Dynamic linear panel regression models with interactive fixed effects. Econometric Theory, pages 1-38.

Moon, H. R. and Weidner, M. (2015). Linear regression for panel with unknown number of factors as interactive fixed effects. Econometrica, 83(4):1543-1579.

O'Brien, G. (1974). The maximum term of uniformly mixing stationary processes. Probability Theory and Related Fields, 30(1):57-63.

Pesaran, M. H. (2006). Estimation and inference in large heterogeneous panels with a multifactor error structure. Econometrica, 74(4):967-1012.

Pinelis, I. (1994). Optimum bounds for the distributions of martingales in banach spaces. The Annals of Probability, pages 1679-1706.

Ramsay, J. O. and Silverman, B. W. (2005). Functional Data Analysis (2nd ED). Springer: New York.

Shang, Z. (2010). Convergence rate and Bahadur type representation of general smoothing spline Mestimates. Electronic Journal of Statistics, 4:1411-1442.

Shang, Z. and Cheng, G. (2013). Local and global asymptotic inference in smoothing spline models. The Annals of Statistics, 41(5):2608-2638.

Shang, Z. and Cheng, G. (2015). Nonparametric inference in generalized functional linear models. The Annals of Statistics, 43(4):1742-1773.

Shao, J. (2003). Mathematical Statistics. Springer: New York.

$\mathrm{Su}, \mathrm{L}$. and Jin, S. (2012). Sieve estimation of panel data models with cross section dependence. Journal of Econometrics, 169(1):34-47.

Su, L., Jin, S., and Zhang, Y. (2015). Specification test for panel data models with interactive fixed effects. Journal of Econometrics, 186(1):222-244.

$\mathrm{Su}$, L. and Zhang, Y. (2013). Nonparametric dynamic panel data models with interactive fixed effects: sieve estimation and specification testing. Working Paper.

Sun, H. (2005). Mercer theorem for RKHS on noncompact sets. Journal of Complexity, 21(3):337-349.

Wahba, G. (1990). Spline models for observational data. SIAM.

Wang, Y. (2011). Smoothing splines: methods and applications. CRC Press.

Weinberger, H. F. (1974). Variational methods for eigenvalue approximation, volume 15. SIAM.

Zhao, T., Cheng, G., and Liu, H. (2016). A partially linear framework for massive heterogeneous data. The Annals of Statistics, 44(4):1400-1437. 
Supplement to

\section{Statistical Inference on Panel Data Models: A Kernel Ridge Regression Method}

This supplement document contains proofs and other relevant results that were not included in the main text and appendix. In Section S.1, we prove Lemmas A.1 and A.2, Propositions A.1 and A.2. In Section S.2, we prove Lemmas A.3, A.4, A.5 A.6, A.7 and A.8. We also provide additional Lemmas S.1, S.2 and S.3 as well as their proofs. Lemmas S.1 and S.2 give mild conditions to guarantee the validity of Assumption A4; Lemma S.3 is useful for proving Lemma A.4.

\section{S.1. Additional Proofs or Other Relevant Results for Heterogeneous Model}

Proof of Lemma A.1. For any $\theta, \theta_{k}=\left(\beta_{k}, g_{k}\right) \in \Theta_{i}$ for $k=1,2$, it holds from (2.1) that

$$
\begin{aligned}
& \left\langle D S_{i, M, \eta_{i}}^{\star}(\theta) \theta_{1}, \theta_{2}\right\rangle_{i} \\
= & \left\langle E\left\{D S_{i, M, \eta_{i}}(\theta) \theta_{1}\right\}, \theta_{2}\right\rangle_{i} \\
= & \frac{1}{T} \sum_{t=1}^{T} E\left(\left\langle R_{i} U_{i t}, \theta_{1}\right\rangle_{i}\left\langle R_{i} U_{i t}, \theta_{2}\right\rangle_{i}\right)+\left\langle P_{i} \theta_{1}, \theta_{2}\right\rangle_{i} \\
= & \frac{1}{T} \sum_{t=1}^{T} E\left(\left(g_{1}\left(X_{i t}\right)+Z_{t}^{\prime} \beta_{1}\right)\left(g_{2}\left(X_{i t}\right)+Z_{t}^{\prime} \beta_{2}\right)\right)+\eta_{i}\left\langle g_{1}, g_{2}\right\rangle_{\mathcal{H}_{i}} \\
= & E\left(\left(g_{1}\left(X_{i}\right)+Z^{\prime} \beta_{1}\right)\left(g_{2}\left(X_{i}\right)+Z^{\prime} \beta_{2}\right)\right)+\eta_{i}\left\langle g_{1}, g_{2}\right\rangle_{\mathcal{H}_{i}} \\
= & \left\langle\theta_{1}, \theta_{2}\right\rangle_{i},
\end{aligned}
$$

which implies that $D S_{i, M, \eta_{i}}^{\star}(\theta)=i d$, the identity operator on $\Theta_{i}$.

Proof of Lemma A.2. It follows by Proposition 2.1 that

$$
\left\|R_{i} U_{i t}\right\|_{i}^{2}=K^{(i)}\left(X_{i t}, X_{i t}\right)+\left(Z_{t}-A_{i}\left(X_{i t}\right)\right)^{\prime}\left(\Omega_{i}+\Sigma_{i}\right)^{-1}\left(Z_{t}-A_{i}\left(X_{i t}\right)\right) .
$$

By $(2.6)$ and $\left\langle A_{i}, g\right\rangle_{\star, i}=V_{i}\left(G_{i}, g\right)$ (see Section 3.2),

$$
\begin{aligned}
A_{i}(x) & =\left\langle A_{i}, K_{x}^{(i)}\right\rangle_{\star, i}=V_{i}\left(G_{i}, K_{x}^{(i)}\right) \\
& =\sum_{\nu=1}^{\infty} \frac{\varphi_{\nu}^{(i)}(x)}{1+\eta_{i} \rho_{\nu}^{(i)}} V_{i}\left(G_{i}, \varphi_{\nu}^{(i)}\right) .
\end{aligned}
$$


It follows by Assumption A3 that $C_{\varphi, i} \equiv \sup _{\nu \geq 1} \sup _{x \in \mathcal{X}_{i}}\left|\varphi_{\nu}^{(i)}(x)\right|<\infty$. Then we have

$$
\begin{aligned}
K^{(i)}\left(X_{i t}, X_{i t}\right) & =\sum_{\nu \geq 1} \frac{\left|\varphi_{\nu}^{(i)}\left(X_{i t}\right)\right|^{2}}{1+\eta_{i} \rho_{\nu}^{(i)}} \leq C_{\varphi, i}^{2} h_{i}^{-1} \\
A_{i}\left(X_{i t}\right)^{\prime}\left(\Omega_{i}+\Sigma_{i}\right)^{-1} A_{i}\left(X_{i t}\right) & \leq c_{1}^{-1} A_{i}\left(X_{i t}\right)^{\prime} A_{i}\left(X_{i t}\right) \\
& \leq c_{1}^{-1} \sum_{\nu \geq 1} \frac{\left|\varphi_{\nu}^{(i)}\left(X_{i}\right)\right|^{2}}{\left(1+\eta_{i} \rho_{\nu}^{(i)}\right)^{2}} \sum_{\nu \geq 1} V_{i}\left(G_{i}^{\prime}, \varphi_{\nu}^{(i)}\right) V_{i}\left(G_{i}, \varphi_{\nu}^{(i)}\right) \\
& \leq c_{1}^{-1} C_{\varphi, i}^{2} C_{G_{i}}^{2} h_{i}^{-1} \\
Z_{t}^{\prime}\left(\Omega_{i}+\Sigma_{i}\right)^{-1} Z_{t} & \leq c_{1}^{-1} Z_{t}^{\prime} Z_{t}
\end{aligned}
$$

where $C_{G_{i}}^{2}=\sum_{\nu \geq 1} V_{i}\left(G_{i}^{\prime}, \varphi_{\nu}^{(i)}\right) V_{i}\left(G_{i}, \varphi_{\nu}^{(i)}\right)$. By Assumption A1, $C_{G_{i}}^{2}$ is a finite positive constant. Then (A.1) holds for $C_{i}^{2}=\max \left\{C_{\varphi, i}^{2}, 2 c_{1}^{-1} C_{\varphi, i}^{2} C_{G_{i}}^{2}, 2 c_{1}^{-1}\right\}$.

To show (A.2), first notice that, for any $\theta=(\beta, g) \in \Theta_{i}$,

$$
\|\theta\|_{i, \text { sup }}=\sup _{x \in \mathcal{X}_{i},\|z\|_{2}=1}\left|g(x)+z^{\prime} \beta\right| .
$$

The " $\geq$ " is obvious. To show " $\leq$ ", note that for any $x \in \mathcal{X}_{i}$, choose $z_{x}=\operatorname{sign}(g(x)) \beta /\|\beta\|_{2}$. Then

$$
\left|g(x)+z_{x}^{\prime} \beta\right|=|g(x)|+\|\beta\|_{2} .
$$

Therefore,

$$
\sup _{x \in \mathcal{X}_{i},\|z\|_{2}=1}\left|g(x)+z^{\prime} \beta\right| \geq \sup _{x \in \mathcal{X}_{i}}\left|g(x)+z_{x}^{\prime} \beta\right|=\sup _{x \in \mathcal{X}_{i}}|g(x)|+\|\beta\|_{2}=\|\theta\|_{i, \text { sup }}
$$

Following Proposition 2.1 and the proof of (A.1), for $u=(x, z)$ with $x \in \mathcal{X}_{i}$ and $\|z\|_{2}=1$,

$$
\left|g(x)+z^{\prime} \beta\right|=\left|\left\langle R_{i} u, \theta\right\rangle_{i}\right| \leq\left\|R_{i} u\right\|_{i}\|\theta\|_{i} \leq C_{i}\left(1+h_{i}^{-1 / 2}\right)\|\theta\|_{i} .
$$

This proves (A.2).

Proof of Proposition A.1. Since $f_{1 t}, f_{2 t}, v_{i t}$ and $\epsilon_{i t}$ all have finite $\alpha$ th moments, it follows by (2.2) that $X_{i t}$ and $Z_{t}$ both have finite $\alpha$ th moments, i.e., $E\left(\left\|X_{i}\right\|_{2}^{\alpha}\right)<\infty$ and $E\left(\|Z\|_{2}^{\alpha}\right)<\infty$. Define

$$
C_{T}(\xi)=\inf \left\{x \mid T P\left(\|Z\|_{2}>x\right) \leq \xi\right\}, \quad \xi>0 .
$$

By Markov inequality,

$$
P\left(\|Z\|_{2}>\left[T E\left(\|Z\|_{2}^{\alpha}\right) / \xi\right]^{1 / \alpha}\right) \leq \frac{\xi}{T}
$$

therefore,

$$
C_{T}(\xi) \leq\left(\frac{T E\left(\|Z\|_{2}^{\alpha}\right)}{\xi}\right)^{1 / \alpha}
$$

Thanks to the $\phi$-mixing condition (see Assumption A2), it follows by O'Brien (1974, Theorem 1) that for any $\xi>0$,

$$
\liminf _{T \rightarrow \infty} P\left(\max _{1 \leq t \leq T}\left\|Z_{t}\right\|_{2} \leq C_{T}(\xi)\right)=\exp (-b \xi)
$$


where $b>0$ is a constant. For arbitrary $\varepsilon>0$, choose $\xi>0$ such that $1-\exp (-b \xi)<\varepsilon / 2$. Then, as $T$ approaches infinity,

$$
P\left(\max _{1 \leq t \leq T}\left\|Z_{t}\right\|_{2} \leq C_{T}(\xi)\right) \geq \exp (-b \xi)-\varepsilon / 2
$$

leading to that

$$
P\left(\max _{1 \leq t \leq T}\left\|Z_{t}\right\|_{2}>C_{T}(\xi)\right) \leq 1-\exp (-b \xi)+\varepsilon / 2 \leq \varepsilon
$$

This proves that

$$
\max _{1 \leq t \leq T}\left\|Z_{t}\right\|_{2}=O_{P}\left(C_{T}(\xi)\right)=O_{P}\left(T^{1 / \alpha}\right)
$$

Proof of Proposition A.2. For notation simplicity, denote

$$
\begin{aligned}
& \mathcal{F}_{j}=\mathcal{F}_{1}^{j}, \quad j \in[T] \\
& \mathcal{F}_{0}=\text { trivial } \sigma \text {-algebra consisting only of the empty set and full sample space. }
\end{aligned}
$$

For any $\theta_{1}, \theta_{2} \in \Theta_{i}$, define $l_{i t}=\left(\psi_{i, M, t}\left(U_{i t} ; \theta_{1}\right)-\psi_{i, M, t}\left(U_{i t} ; \theta_{2}\right)\right) R_{i} U_{i t}$. First of all, we will prove the following concentration inequality: for any $r>0$,

$$
P\left(\left\|\sum_{t=1}^{T}\left[l_{i t}-E\left(l_{i t}\right)\right]\right\|_{i} \geq r\right) \leq 2 \exp \left(-\frac{r^{2}}{32 T C_{\phi}^{2}\left\|\theta_{1}-\theta_{2}\right\|_{i, \text { sup }}^{2}}\right),
$$

where $C_{\phi} \equiv \sum_{t=0}^{\infty} \phi(t)$. It follows by Assumption A2 that $C_{\phi}$ is finite. Clearly, (S.1) holds for $\theta_{1}=\theta_{2}$ since both sides equal to zero. In what follows, we assume $\theta_{1} \neq \theta_{2}$.

Define $\mathbb{M}_{i T}=\sum_{t=1}^{T} l_{i t}$, and $f_{i T j}=E\left(\mathbb{M}_{i T} \mid \mathcal{F}_{j}\right)-E\left(\mathbb{M}_{i T} \mid \mathcal{F}_{j-1}\right), j \in[T]$. It is easy to see that

$$
\begin{aligned}
\mathbb{M}_{i T}-E\left(\mathbb{M}_{i T}\right) & =\sum_{j=1}^{T} f_{i T j} \\
f_{i T j} & =\sum_{t=j}^{T}\left(E\left(l_{i t} \mid \mathcal{F}_{j}\right)-E\left(l_{i t} \mid \mathcal{F}_{j-1}\right)\right) .
\end{aligned}
$$

Clearly, $f_{i T j}$ is $\mathcal{F}_{j}$-measurable. For $k \in[T]$, define $\mathbb{N}_{i T k}=\sum_{j=1}^{k} f_{i T j}$ and $\mathbb{N}_{i T 0} \equiv 0$. Then $\mathbb{N}_{i T k}=$ $\mathbb{N}_{i T k-1}+f_{i T k}$. For $\lambda>0$, let $u_{k-1}(x)=\lambda\left\|\mathbb{N}_{i T k-1}+x f_{i T k}\right\|_{i}, x \in[0,1]$. Define

$$
\varphi_{k-1}(x)=E\left(\cosh \left(u_{k-1}(x)\right) \mid \mathcal{F}_{k-1}\right), x \in[0,1]
$$

It is easy to see that

$$
\begin{aligned}
\varphi_{k-1}(1) & =E\left(\cosh \left(\lambda\left\|\mathbb{N}_{i T k}\right\|_{i}\right) \mid \mathcal{F}_{k-1}\right) \\
\varphi_{k-1}(0) & =E\left(\cosh \left(\lambda\left\|\mathbb{N}_{i T k-1}\right\|_{i}\right) \mid \mathcal{F}_{k-1}\right) .
\end{aligned}
$$


By the proof of Pinelis (1994, Theorem 3.2) and direct calculations, it can be shown that

$$
\begin{aligned}
\varphi_{k-1}^{\prime}(x) & =E\left(\sinh \left(u_{k-1}(x)\right) u_{k-1}^{\prime}(x) \mid \mathcal{F}_{k-1}\right) \\
\varphi_{k-1}^{\prime \prime}(x) & =E\left(\cosh \left(u_{k-1}\right)\left(u_{k-1}^{\prime}(x)\right)^{2}+\sinh \left(u_{k-1}(x)\right) u_{k-1}^{\prime \prime}(x) \mid \mathcal{F}_{k-1}\right) \\
& \leq E\left(\cosh \left(u_{k-1}\right)\left(u_{k-1}^{\prime}(x)\right)^{2}+\cosh \left(u_{k-1}(x)\right) u_{k-1}(x) u_{k-1}^{\prime \prime}(x) \mid \mathcal{F}_{k-1}\right) \\
& =\frac{1}{2} E\left(\cosh \left(u_{k-1}(x)\right)\left(u_{k-1}(x)^{2}\right)^{\prime \prime} \mid \mathcal{F}_{k-1}\right) \\
& =\lambda^{2} E\left(\cosh \left(u_{k-1}(x)\right)\left\|f_{i T k}\right\|_{i}^{2} \mid \mathcal{F}_{k-1}\right) .
\end{aligned}
$$

Next we will show that $\left\|f_{i T k}\right\|_{i}^{2}$ is almost surely bounded. We will first examine the terms $E\left(l_{i t} \mid \mathcal{F}_{k}\right)-E\left(l_{i t}\right)$ for $t \geq k$. Arbitrarily choose $A \in \mathcal{F}_{k}$ and $\theta \in \Theta_{i}$ with $\|\theta\|_{i}=1$. Define $X=\left\langle l_{i t}, \theta\right\rangle_{i}$. Write $X=X^{+}-X^{-}$, where $X^{+}$and $X^{-}$represent the positive and negative parts of $X$, respectively. Clearly, $|X| \leq\left\|l_{i t}\right\|_{i}\|\theta\|_{i}=\left\|l_{i t}\right\|_{i}$ implying that both $X^{+}$and $X^{-}$belong to $\left[0,\left\|l_{i t}\right\|_{i}\right]$. Note that the $X^{+}$is $\mathcal{F}_{t}^{\infty}$-measurable. Therefore,

$$
\begin{aligned}
\left|E\left(X^{+} \mid A\right)-E\left(X^{+}\right)\right| & =\left|\int_{0}^{\left\|l_{i t}\right\|_{i}}\left[P\left(X^{+}>v \mid A\right)-P\left(X^{+}>v\right)\right] d v\right| \\
& \leq \int_{0}^{\left\|l_{i t}\right\|_{i}}\left|P\left(X^{+}>v \mid A\right)-P\left(X^{+}>v\right)\right| d v \leq\left\|l_{i t}\right\|_{i} \phi(t-k) .
\end{aligned}
$$

Similarly, one can show that $\left|E\left(X^{-} \mid A\right)-E\left(X^{-}\right)\right| \leq\left\|l_{i t}\right\|_{i} \phi(t-k)$. Therefore,

$$
|E(X \mid A)-E(X)| \leq 2\left\|l_{i t}\right\|_{i} \phi(t-k) .
$$

By arbitrariness of $A \in \mathcal{F}_{k}$ and by taking supremum over $\theta \in \Theta_{i}$ with $\|\theta\|_{i}=1$, one gets that

$$
\left\|E\left(l_{i t} \mid \mathcal{F}_{k}\right)-E\left(l_{i t}\right)\right\|_{i} \leq 2\left\|l_{i t}\right\|_{i} \phi(t-k), t \geq k .
$$

Similar arguments lead to

$$
\left\|E\left(l_{i t} \mid \mathcal{F}_{k-1}\right)-E\left(l_{i t}\right)\right\|_{i} \leq 2\left\|l_{i t}\right\|_{i} \phi(t-k+1), t \geq k .
$$

Therefore, for $t \geq k$,

$$
\left\|E\left(l_{i t} \mid \mathcal{F}_{k}\right)-E\left(l_{i t} \mid \mathcal{F}_{k-1}\right)\right\|_{i} \leq 2\left\|l_{i t}\right\|_{i}(\phi(t-k)+\phi(t-k+1)) .
$$

Using (S.2) and the assumption $\left\|l_{i t}\right\|_{i} \leq\left\|\theta_{1}-\theta_{2}\right\|_{i \text {,sup }}$, it can be shown that

$$
\begin{aligned}
\left\|f_{i T k}\right\|_{i} & \leq \sum_{t=k}^{T}\left\|E\left(l_{i t} \mid \mathcal{F}_{k}\right)-E\left(l_{i t} \mid \mathcal{F}_{k-1}\right)\right\|_{i} \\
& \leq \sum_{t=k}^{T} 2\left\|l_{i t}\right\|_{i}(\phi(t-k)+\phi(t-k+1)) \\
& \leq 2\left\|\theta_{1}-\theta_{2}\right\|_{i, \text { sup }} \sum_{t=k}^{T}(\phi(t-k)+\phi(t-k+1)) \\
& \leq 4 C_{\phi}\left\|\theta_{1}-\theta_{2}\right\|_{i, \text { sup }} .
\end{aligned}
$$


Therefore, it follows by (S.3) that

$$
\varphi_{k-1}^{\prime \prime}(x) \leq 16 \lambda^{2} C_{\phi}^{2}\left\|\theta_{1}-\theta_{2}\right\|_{i, \text { sup }}^{2} \varphi_{k-1}(x) .
$$

Meanwhile, note that $\mathbb{N}_{i T k-1}$ is $\mathcal{F}_{k-1}$-measurable, so we have

$$
\varphi_{k-1}^{\prime}(0)=\lambda \frac{\sinh \left(\lambda\left\|\mathbb{N}_{i T k-1}\right\|_{i}\right)}{\left\|\mathbb{N}_{i T k-1}\right\|_{i}} E\left(\left\langle\mathbb{N}_{i T k-1}, f_{i T k}\right\rangle_{i} \mid \mathcal{F}_{k-1}\right)=0, k \geq 2,
$$

where the last equality follows from $E\left(f_{i T k} \mid \mathcal{F}_{k-1}\right)=0$. Directly using (S.3) one also has that $\varphi_{0}^{\prime}(0)=0$. So $\varphi_{k-1}^{\prime}(0)=0$ for all $k \in[T]$. By Dudley et al. (1992, pp. 133, Lemma 3) we have for $k \in[T]$,

$$
\varphi_{k-1}(x) \leq \varphi_{k-1}(0) \exp \left(8 \lambda^{2} C_{\phi}^{2}\left\|\theta_{1}-\theta_{2}\right\|_{i, \mathrm{sup}}^{2} x^{2}\right), \quad x \in[0,1] .
$$

In particular,

$$
\varphi_{k-1}(1) \leq \varphi_{k-1}(0) \exp \left(8 \lambda^{2} C_{\phi}^{2}\left\|\theta_{1}-\theta_{2}\right\|_{i, \text { sup }}^{2}\right) .
$$

Taking expectations on both sides leading to that

$$
E\left(\cosh \left(\lambda\left\|\mathbb{N}_{i T k}\right\|_{i}\right)\right) \leq \exp \left(8 \lambda^{2} C_{\phi}^{2}\left\|\theta_{1}-\theta_{2}\right\|_{i, \sup }^{2}\right) E\left(\cosh \left(\lambda\left\|\mathbb{N}_{i T k-1}\right\|_{i}\right)\right) .
$$

By repeatedly using (S.5) and the convention $\mathbb{N}_{i T 0}=0$, and by (S.2), we have

$$
\begin{aligned}
E\left(\cosh \left(\lambda\left\|\sum_{t=1}^{T}\left[l_{i t}-E\left(l_{i t}\right)\right]\right\|_{i}\right)\right) & =E\left(\cosh \left(\lambda\left\|\mathbb{N}_{i T T}\right\|_{i}\right)\right) \\
& \leq \exp \left(8 T \lambda^{2} C_{\phi}^{2}\left\|\theta_{1}-\theta_{2}\right\|_{i, \text { sup }}^{2}\right) .
\end{aligned}
$$

Therefore,

$$
\begin{aligned}
P\left(\left\|\sum_{t=1}^{T}\left[l_{i t}-E\left(l_{i t}\right)\right]\right\|_{i} \geq r\right) & =P\left(\cosh \left(\lambda\left\|\sum_{t=1}^{T}\left[l_{i t}-E\left(l_{i t}\right)\right]\right\|_{i}\right) \geq \cosh (\lambda r)\right) \\
& \leq \frac{1}{\cosh (\lambda r)} E\left(\cosh \left(\lambda\left\|\sum_{t=1}^{T}\left[l_{i t}-E\left(l_{i t}\right)\right]\right\|_{i}\right)\right) \\
& \leq e \exp \left(-\lambda r+8 T \lambda^{2} C_{\phi}^{2}\left\|\theta_{1}-\theta_{2}\right\|_{i, \text { sup }}^{2}\right) .
\end{aligned}
$$

Then (S.1) follows by choosing

$$
\lambda=\frac{r}{16 T C_{\phi}^{2}\left\|\theta_{1}-\theta_{2}\right\|_{i, \text { sup }}^{2}} .
$$

The rest of the proof follows by chaining argument. Let $\psi_{2}(x)=\exp \left(x^{2}\right)-1$. It follows by (S.1) and Kosorok (2008, Theorem 8.1) that for any $\theta_{1}, \theta_{2} \in \Theta_{i}$,

$$
\|\| \mathbb{Z}_{i M}\left(\theta_{1}\right)-\mathbb{Z}_{i M}\left(\theta_{2}\right)\left\|_{i}\right\|_{\psi_{2}} \leq \sqrt{96} C_{\phi}\left\|\theta_{1}-\theta_{2}\right\|_{i, \text { sup }}
$$


It follows by Kosorok (2008, Theorem 8.4) that there exists a universal constant $C>0$, which only depends on $C_{\psi}$, such that for any $\delta>0$,

$$
\begin{aligned}
& \left\|\sup _{\substack{\theta_{1}, \theta_{2} \in \mathcal{G}_{i}\left(p_{i}\right) \\
\left\|\theta_{1}-\theta_{2}\right\|_{i, \text { sup }} \leq \delta}}\right\| \mathbb{Z}_{i M}\left(\theta_{1}\right)-\mathbb{Z}_{i M}\left(\theta_{2}\right)\left\|_{i}\right\|_{\psi_{2}} \\
\leq & C\left(\int_{0}^{\delta} \psi_{2}^{-1}\left(D_{i}\left(\varepsilon, \mathcal{G}_{i}\left(p_{i}\right),\|\cdot\|_{i, \text { sup }}\right)\right) d \varepsilon+\delta \psi_{2}^{-1}\left(D_{i}\left(\delta, \mathcal{G}_{i}\left(p_{i}\right),\|\cdot\|_{i, \text { sup }}\right)^{2}\right)\right)=C J_{i}\left(p_{i}, \delta\right) .
\end{aligned}
$$

Therefore,

$$
\left\|\sup _{\substack{\theta \in \mathcal{G}_{i}\left(p_{i}\right) \\\|\theta\|_{i, \text { sup }} \leq \delta}}\right\| \mathbb{Z}_{i M}(\theta)\left\|_{i}\right\|_{\psi_{2}} \leq C J_{i}\left(p_{i}, \delta\right) .
$$

It follows again from Kosorok (2008, Lemma 8.1) that for all $\delta>0, s>0$,

$$
P\left(\sup _{\theta \in \mathcal{G}_{i}\left(p_{i}\right),\|\theta\|_{i, \text { sup }} \leq \delta}\left\|\mathbb{Z}_{i M}(\theta)\right\|_{i}>s\right) \leq 2 \exp \left(-\frac{s^{2}}{C^{2} J_{i}\left(p_{i}, \delta\right)^{2}}\right) .
$$

It is easy to see that for any $\theta \in \mathcal{G}_{i}\left(p_{i}\right),\|\theta\|_{i \text {,sup }} \leq 1$. Let $\sqrt{T} J_{i}\left(p_{i}, 1\right)=\varepsilon^{-1}$, and $Q_{\varepsilon}=-\log \varepsilon-1$. Let $\tau=3 C \sqrt{\log N+\log \log \left(T J_{i}\left(p_{i}, 1\right)\right)}$. Then it can be checked that

$$
N\left(Q_{\varepsilon}+2\right) \exp \left(-\frac{\tau^{2}}{C^{2} \exp (2)}\right) \rightarrow 0, \quad N \rightarrow \infty
$$

Since $J_{i}\left(p_{i}, \delta\right)$ is strictly increasing in $\delta$, the function $J_{i}(\delta) \equiv J_{i}\left(p_{i}, \delta\right)$ has inverse denoted by $J_{i}^{-1}$. Then we have

$$
\begin{aligned}
& P\left(\max _{i \in[N]} \sup _{\theta \in \mathcal{G}_{i}\left(p_{i}\right)} \frac{\sqrt{T}\left\|\mathbb{Z}_{i M}(\theta)\right\|_{i}}{\sqrt{T} J_{i}\left(\|\theta\|_{i, \text { sup }}\right)+1} \geq \tau\right) \\
\leq & \sum_{i=1}^{N}\left(P\left(\sup _{\|\theta\|_{i, \text { sup }} \leq J_{i}^{-1}\left(T^{-1 / 2}\right)} \frac{\sqrt{T}\left\|\mathbb{Z}_{i M}(\theta)\right\|_{i}}{\sqrt{T} J_{i}\left(\|\theta\|_{i, \text { sup }}\right)+1} \geq \tau\right)\right. \\
& \left.+\sum_{l=0}^{Q_{\varepsilon}} P\left(\sup _{J_{i}^{-1}\left(T^{-1 / 2} \exp (l)\right) \leq\|\theta\|_{i, \text { sup }} \leq J_{i}^{-1}\left(T^{-1 / 2} \exp (l+1)\right)} \frac{\sqrt{T}\left\|\mathbb{Z}_{i M}(\theta)\right\|_{i}}{\sqrt{T} J_{i}\left(\|\theta\|_{i, \text { sup }}\right)+1} \geq \tau\right)\right) \\
\leq & \sum_{i=1}^{N}\left(P\left(\sup _{\|\theta\|_{i, \text { sup }} \leq J_{i}^{-1}\left(T^{-1 / 2}\right)}\left\|\mathbb{Z}_{i M}(\theta)\right\|_{i} \geq T^{-1 / 2} \tau\right)\right. \\
& \left.+\sum_{l=0}^{Q_{\varepsilon}} P\left(\sup _{\|\theta\|_{i, \sup } \leq J_{i}^{-1}\left(T^{-1 / 2} \exp (l+1)\right)}\left\|\mathbb{Z}_{i M}(\theta)\right\|_{i} \geq T^{-1 / 2} \tau \exp (l)\right)\right) \\
\leq & \sum_{i=1}^{N}\left(2 \exp \left(-\tau^{2} / C^{2}\right)+\sum_{l=0}^{Q_{\varepsilon}} 2 \exp \left(-\tau^{2} /\left(C^{2} \exp (2)\right)\right)\right) \\
= & 2 N\left(Q_{\varepsilon}+2\right) \exp \left(-\frac{\tau^{2}}{C^{2} \exp (2)}\right) \rightarrow 0, \quad \text { as } N \rightarrow \infty .
\end{aligned}
$$

This proves the desirable conclusion with $C_{0}=3 C$. 


\section{S.2. Additional Proofs or Other Relevant Results for Homogeneous Model}

The following lemma gives mild conditions that guarantee Assumption A4. Before stating the lemma, we borrow the concept of complete continuity from Weinberger (1974, page 50). A bilinear functional $A(\cdot, \cdot)$ on $\mathcal{H} \times \mathcal{H}$ is said to be completely continuous w.r.t another bilinear functional $B(\cdot, \cdot)$ if for any $\epsilon>0$, there exists finite number of functionals $l_{1}, l_{2}, \ldots, l_{k}$ on $\mathcal{H}$ such that $l_{i}(g)=0, i=1,2, . ., k$, implies $A(g, g) \leq \epsilon B(g, g)$.

Let $U$ be an open subset of $\mathcal{X}$ and $U^{N T} \equiv \underbrace{U \times U \times \cdots \times U}_{N T \text { items }}$. Let $C(\mathcal{X})$ be the set of all continuous functions on $\mathcal{X}$ and $\mathcal{H} \subseteq C(\mathcal{X})$. Let $\mathbf{x}$ denote the $N T$-vector $\left(x_{11}, \ldots, x_{1 T}, \ldots, x_{N 1}, \ldots, x_{N T}\right)$.

Lemma S.1. Suppose $1 \notin \mathcal{H}$, and $p\left(\mathbf{x} \mid \mathcal{F}_{1}^{T}\right)>0$ for $\mathbf{x} \in U^{N T}$, where $p\left(\mathbf{x} \mid \mathcal{F}_{1}^{T}\right)$ is the joint conditional density of $X_{11}, X_{12}, \ldots, X_{N T}$ given $\mathcal{F}_{1}^{T}$. If $V(f, g)=0$ for all $f \in \mathcal{H}$, then $g=0$.

Proof of Lemma S.1. For simplicity, we assume that $f_{1 t}, X_{i t}$ are both univariate. By assumption, $0=V(g, g)=\sum_{i=1}^{N} E\left\{\left(\tau_{i} g\right)^{\prime} P\left(\tau_{i} g\right) \mid \mathcal{F}_{1}^{T}\right\} /(N T)$. Hence it follows that

$$
0=\int\left(g\left(x_{i 1}\right), g\left(x_{i 2}\right), \ldots, g\left(x_{i T}\right)\right) P\left(g\left(x_{i 1}\right), g\left(x_{i 2}\right), \ldots, g\left(x_{i T}\right)\right)^{\prime} p\left(\mathbf{x} \mid \mathcal{F}_{1}^{T}\right) d \mathbf{x}, \text { for all } i \in[N] .
$$

Since the integrand in (S.1) is continuous and nonnegative, it holds that, for all $i \in[N]$ and $\mathbf{x}$ with $p\left(\mathbf{x} \mid \mathcal{F}_{1}^{T}\right)>0$,

$$
\left(g\left(x_{i 1}\right), g\left(x_{i 2}\right), \ldots, g\left(x_{i T}\right)\right) P\left(g\left(x_{i 1}\right), g\left(x_{i 2}\right), \ldots, g\left(x_{i T}\right)\right)^{\prime}=0 .
$$

By definition, $P$ is a projection matrix whose image is the orthogonal space of the linear space spanned by $F_{1}$ and $\bar{X}$. Therefore, it yields that

$$
\left(g\left(x_{i 1}\right), g\left(x_{i 2}\right), \ldots, g\left(x_{i T}\right)\right)=\alpha_{i}\left(f_{11}, f_{12}, \ldots, f_{1 T}\right)+\beta_{i}\left(\bar{x}_{1}, \bar{x}_{2}, \ldots, \bar{x}_{T}\right)
$$

for some $\alpha_{i}, \beta_{i} \in \mathbb{R}$. Consider $\mathbf{x}=\left(x_{11}, x_{12}, \ldots, x_{N, T-1}, x_{N T}\right)$ and $\widetilde{\mathbf{x}}=\left(x_{11}, x_{12}, \ldots, x_{N, T-1}, \widetilde{x}_{N T}\right) \in$ $U^{N T}$ with $x_{N T} \neq \widetilde{x}_{N T}$ and $p\left(\mathbf{x} \mid \mathcal{F}_{1}^{T}\right)>0, p\left(\widetilde{\mathbf{x}} \mid \mathcal{F}_{1}^{T}\right)>0$, i.e., the two points differ only on the last element. Applying (S.2) to point $\widetilde{\mathbf{x}}$, we have

$$
\left(g\left(x_{i 1}\right), g\left(x_{i 2}\right), \ldots, g\left(\widetilde{x}_{i T}\right)\right)=\widetilde{\alpha}_{i}\left(f_{11}, f_{12}, \ldots, f_{1 T}\right)+\widetilde{\beta}_{i}\left(\bar{x}_{1}, \bar{x}_{2}, \ldots, \widetilde{\bar{x}}_{T}\right),
$$

for some $\widetilde{\alpha}_{i}, \widetilde{\beta}_{i} \in \mathbb{R}$. Comparing (S.3) and (S.4), and by the fact $T>q_{1}+d=2$, it holds that $\alpha_{i}=\widetilde{\alpha}_{i}, \beta_{i}=\widetilde{\beta}_{i}=0$. Hence $\left(g\left(x_{i 1}\right), g\left(x_{i 2}\right), \ldots, g\left(x_{i T}\right)\right) \in \operatorname{span}\left(\left(f_{11}, f_{12}, \ldots, f_{1 T}\right)\right)$ for all $p\left(\mathbf{x} \mid \mathcal{F}_{1}^{T}\right)>0, i \in[N]$, and it happens if and only if $g=0$.

Lemma S.2. Suppose $\mathcal{X}$ is compact. Furthermore if $V(f, g)=0$ for all $f \in \mathcal{H}$ implies $g=0$, then Assumption $A_{4}$ is valid.

Remark S.2.1. The compactness of $\mathcal{X}$ can be relaxed by Mercer's theorem; see Sun (2005). 
Proof of Lemma S.2. Define bilinear functionals $W(g, \widetilde{g})=\sum_{i=1}^{N} E\left\{\left(\tau_{i} g\right)^{\prime}\left(\tau_{i} \widetilde{g}\right) \mid \mathcal{F}_{1}^{T}\right\} /(N T)$, and $J(g, \widetilde{g})=\langle g, \widetilde{g}\rangle_{\mathcal{H}}$. Clearly, $V(g, g) \leq W(g, g)$. Let $\mu$ be a measure such that

$$
\int g d \mu=\frac{1}{N T} \sum_{i=1}^{N} \sum_{t=1}^{T} E\left(g\left(X_{i t}\right) \mid \mathcal{F}_{1}^{T}\right) .
$$

Hence, $\int g^{2} d \mu=W(g, g)$. By Mercer's theorem, the kernel $\bar{K}$ of $\mathcal{H}$ follows the expansion:

$$
\bar{K}(x, y)=\sum_{i=1}^{\infty} \lambda_{i} e_{i}(x) e_{i}(y)
$$

where $\lambda_{i}$ is a non-increasing positive sequence converging to zero and $\left\{e_{i}\right\}_{i=1}^{\infty}$ forms an orthonormal basis of $L_{2}(\mu)$, so that $W\left(e_{i}, e_{j}\right)=\delta_{i j}$. Moreover, $\left\{\sqrt{\lambda_{i}} e_{i}\right\}_{i=1}^{\infty}$ is also an orthonormal basis of $\mathcal{H}$, which is proved in Cucker and Smale (2001). As a consequence, any $g \in \mathcal{H}$ simultaneously admits the following expansions:

$$
g=\sum_{i=1}^{\infty} W\left(g, e_{i}\right) e_{i}, \quad g=\sum_{i=1}^{\infty} J\left(g, \sqrt{\lambda_{i}} e_{i}\right) \sqrt{\lambda_{i}} e_{i}
$$

with $\sum_{i=1}^{\infty} W^{2}\left(g, e_{i}\right)<\infty$ and $\sum_{i=1}^{\infty} J^{2}\left(g, \sqrt{\lambda_{i}} e_{i}\right)<\infty$. This implies $W\left(g, e_{i}\right)=\lambda_{i} J\left(g, e_{i}\right)$. For any $\epsilon>0$, choose integer $k$ large enough so that $\lambda_{i}<\epsilon$ for $i>k$. Define functionals $l_{i}(g)=$ $W\left(g, e_{i}\right), i=1,2, \ldots, k$. By direct direct examinations, if $l_{i}(g)=0$ for $i=1,2, \ldots, k$, then

$$
W(g, g)=\sum_{i=k+1}^{\infty} W^{2}\left(g, e_{i}\right)=\sum_{i=k+1}^{\infty} \lambda_{i}^{2} J^{2}\left(g, e_{i}\right) \leq \epsilon \sum_{i=k+1}^{\infty} \lambda_{i} J^{2}\left(g, e_{i}\right)=\epsilon J(g, g) .
$$

Since $V(g, g) \leq W(g, g) \leq \epsilon J(g, g), V$ is completely continuous w.r.t $J$. By Weinberger (1974, Theorem 3.1, page 52), there are positive eigenvalues $\left\{\alpha_{i}\right\}_{i=1}^{\infty}$ converging to zero and eigenfunctions $\left\{\widetilde{\varphi}_{i}\right\}_{i=1}^{\infty} \in \mathcal{H}$ such that $V\left(\widetilde{\varphi}_{i}, \widetilde{\varphi}_{j}\right)=\alpha_{i} \delta_{i j}, J\left(\widetilde{\varphi}_{i}, \widetilde{\varphi}_{j}\right)=\delta_{i j}$ and

$$
g=\sum_{i=1}^{\infty} J\left(g, \widetilde{\varphi}_{i}\right) \widetilde{\varphi}_{i}, \text { for all } g \in \mathcal{H}
$$

The above implies $V\left(g, \widetilde{\varphi}_{i}\right)=\alpha_{i} J\left(g, \widetilde{\varphi}_{i}\right)$. Take $\varphi_{i}=\widetilde{\varphi}_{i} / \sqrt{\alpha_{i}}$ and $\rho_{i}=1 / \alpha_{i}$, then $\left\{\varphi_{i}\right\}_{i=1}^{\infty}$ and $\left\{\rho_{i}\right\}_{i=1}^{\infty}$ will satisfy Assumption A4.

Proof of Lemma A.3. Throughout we let $\|A\|_{F}=\sqrt{\operatorname{Tr}\left(A A^{\prime}\right)}$ be the Frobenius norm. Clearly,

$$
\widetilde{\Sigma}=\left(\begin{array}{cccc}
0 & 0 & \cdots & 0 \\
\bar{v}_{1} & \bar{v}_{2} & \cdots & \bar{v}_{T}
\end{array}\right), \Sigma_{\star} \widetilde{\Sigma}^{\prime}=\left(0_{\left(q_{1}+d\right) \times q_{1}}, \sum_{t=1}^{T} Z_{t}^{\star} \bar{v}_{t}^{\prime}\right) .
$$

By direct examinations we have

$$
\Sigma \Sigma^{\prime}-\Sigma_{\star} \Sigma_{\star}^{\prime}=\Sigma_{\star} \widetilde{\Sigma}^{\prime}+\widetilde{\Sigma}^{\prime} \Sigma_{\star}+\widetilde{\Sigma} \widetilde{\Sigma}^{\prime} \equiv R
$$


By independence of $v_{i t}$ and $Z_{t}^{\star}$, it can be shown that

$$
\begin{aligned}
& E\left(\left\|\sum_{t=1}^{T} Z_{t}^{\star} \bar{v}_{t}^{\prime}\right\|_{F}^{2}\right)=\sum_{t, l=1}^{T} \operatorname{Tr}\left(E\left(\bar{v}_{t}^{\prime} \bar{v}_{l}\right) E\left(\left(Z_{t}^{\star}\right)^{\prime} Z_{l}^{\star}\right)\right)=O(T / N), \\
& E\left(\left\|\widetilde{\Sigma} \widetilde{\Sigma}^{\prime}\right\|_{F}^{2}\right) \leq E\left(\operatorname{Tr}\left(\widetilde{\Sigma} \widetilde{\Sigma}^{\prime} \widetilde{\Sigma}^{\prime} \widetilde{\Sigma}^{\prime}\right)\right)=O\left(T^{2} / N^{2}\right) .
\end{aligned}
$$

Hence,

$$
\begin{aligned}
E\left(\left\|\Sigma_{\star} \widetilde{\Sigma}^{\prime}\right\|_{F}^{2}\right) & =E\left(\left\|\sum_{t=1}^{T} Z_{t}^{\star} \bar{v}_{t}^{\prime}\right\|_{F}^{2}\right)=O(T / N) \\
E\left(\|R\|_{F}^{2}\right) & \leq 8 E\left(\left\|\Sigma_{\star} \widetilde{\Sigma}^{\prime}\right\|_{F}^{2}\right)+2 E\left(\left\|\widetilde{\Sigma} \widetilde{\Sigma}^{\prime}\right\|_{F}^{2}\right)=O\left(T / N+(T / N)^{2}\right) .
\end{aligned}
$$

Since

$$
\begin{aligned}
\left\|\left(\Sigma \Sigma^{\prime}\right)^{-1}-\left(\Sigma_{\star} \Sigma_{\star}^{\prime}\right)^{-1}\right\|_{\mathrm{op}} & =\left\|\left(\Sigma_{\star} \Sigma_{\star}^{\prime}\right)^{-1} R\left(\Sigma \Sigma^{\prime}\right)^{-1}\right\|_{\mathrm{op}} \\
& \leq\left\|\left(\Sigma_{\star} \Sigma_{\star}^{\prime}\right)^{-1}\right\|_{\mathrm{op}}\|R\|_{\mathrm{op}}\left\|\left(\Sigma \Sigma^{\prime}\right)^{-1}\right\|_{\mathrm{op}},
\end{aligned}
$$

it follows by Assumption A5 and (S.5) and Hölder inequality that

$$
E\left(\left\|\left(\Sigma \Sigma^{\prime}\right)^{-1}-\left(\Sigma_{\star} \Sigma_{\star}^{\prime}\right)^{-1}\right\|_{\text {op }}^{1+\omega}\right)=O\left(\left(T^{3} N\right)^{-(1+\omega) / 2}+(T N)^{-(1+\omega)}\right),
$$

where $\omega=(\zeta-4) /(\zeta+4)$. Note that $E\left(\widetilde{\Sigma}^{\prime} \widetilde{\Sigma}\right)=\sigma_{v}^{2} I_{T}$ and $E \operatorname{Tr}\left(\widetilde{\Sigma}^{\prime} \widetilde{\Sigma}\right)=O(T / N)$, where $\sigma_{v}^{2}=$ $E\left(v_{i t}^{\prime} v_{i t}\right)$ is a constant. By direct examinations

$$
\begin{aligned}
& P_{\star}-P \\
= & \Sigma^{\prime}\left(\left(\Sigma \Sigma^{\prime}\right)^{-1}-\left(\Sigma_{\star} \Sigma_{\star}^{\prime}\right)^{-1}\right) \Sigma+\Sigma_{\star}^{\prime}\left(\Sigma_{\star} \Sigma_{\star}^{\prime}\right)^{-1} \widetilde{\Sigma}+\widetilde{\Sigma}^{\prime}\left(\Sigma_{\star} \Sigma_{\star}^{\prime}\right)^{-1} \Sigma_{\star}+\widetilde{\Sigma}^{\prime}\left(\Sigma_{\star} \Sigma_{\star}^{\prime}\right)^{-1} \widetilde{\Sigma} .
\end{aligned}
$$

It follows by (S.6), (S.7) and (S.8) and Hölder inequality that

$$
\begin{aligned}
& E\left(\left\|P-P_{\star}\right\|_{\mathrm{op}}\right) \\
\leq & E\left(\left\|\Sigma \Sigma^{\prime}\right\|_{\mathrm{op}}\left\|\left(\Sigma \Sigma^{\prime}\right)^{-1}-\left(\Sigma_{\star} \Sigma_{\star}^{\prime}\right)^{-1}\right\|_{\mathrm{op}}\right)+2 E\left(\left\|\widetilde{\Sigma}^{\prime}\left(\Sigma_{\star} \Sigma_{\star}^{\prime}\right)^{-1} \Sigma_{\star}\right\|_{\mathrm{op}}\right)+E\left(\left\|\widetilde{\Sigma}^{\prime}\left(\Sigma_{\star} \Sigma_{\star}^{\prime}\right)^{-1} \widetilde{\Sigma}\right\|_{\mathrm{op}}\right) \\
\leq & E\left(\left\|\left(\Sigma \Sigma^{\prime}\right)^{-1}-\left(\Sigma_{\star} \Sigma_{\star}^{\prime}\right)^{-1}\right\|_{\mathrm{op}}^{1+\omega}\right)^{1 /(1+\omega)} E\left(\left\|\Sigma \Sigma^{\prime}\right\|_{\mathrm{op}}^{(1+\omega) / \omega}\right)^{\omega /(1+\omega)} \\
& +2 E\left(\left\|\left(\Sigma_{\star} \Sigma_{\star}^{\prime}\right)^{-1}\right\|_{\mathrm{op}}\right)^{1 / 2} E\left(\operatorname{Tr}\left(\widetilde{\Sigma} \widetilde{\Sigma}^{\prime}\right)\right)^{1 / 2}+E\left(\left\|\left(\Sigma_{\star} \Sigma_{\star}^{\prime}\right)^{-1}\right\|_{\mathrm{op}}\right) E\left(\operatorname{Tr}\left(\widetilde{\Sigma} \widetilde{\Sigma}^{\prime}\right)\right) \\
= & O\left(\left(T^{3} N\right)^{-1 / 2}+(T N)^{-1}\right) T+O\left(T^{-1 / 2} \sqrt{T / N}\right)+O\left(T^{-1}(T / N)\right)=O\left(N^{-1 / 2}\right) .
\end{aligned}
$$

This proves (A.20). Next we show (A.21). For any $i \in[N]$,

$$
\begin{aligned}
& E\left\{\gamma_{2 i}^{\prime} F_{2}^{\prime}\left(P-P_{\star}\right) K_{\mathbb{X}_{i}} \mid \mathcal{F}_{1}^{T}\right\} \\
= & E\left\{\gamma_{2 i}^{\prime} F_{2}^{\prime} \Sigma^{\prime}\left[\left(\Sigma \Sigma^{\prime}\right)^{-1}-\left(\Sigma_{\star} \Sigma_{\star}^{\prime}\right)^{-1}\right] \Sigma K_{\mathcal{X}_{i}} \mid \mathcal{F}_{1}^{T}\right\}+E\left\{\gamma_{2 i}^{\prime} F_{2}^{\prime} \Sigma_{\star}^{\prime}\left(\Sigma_{\star} \Sigma_{\star}^{\prime}\right)^{-1} \widetilde{\Sigma} K_{\mathcal{X}_{i}} \mid \mathcal{F}_{1}^{T}\right\} \\
& +E\left\{\gamma_{2 i}^{\prime} F_{2}^{\prime} \widetilde{\Sigma}^{\prime}\left(\Sigma_{\star} \Sigma_{\star}^{\prime}\right)^{-1} \Sigma_{\star} K_{\mathbb{X}_{i}} \mid \mathcal{F}_{1}^{T}\right\}+E\left\{\gamma_{2 i}^{\prime} F_{2}^{\prime} \widetilde{\Sigma}^{\prime}\left(\Sigma_{\star} \Sigma_{\star}^{\prime}\right)^{-1} \widetilde{\Sigma} K_{\mathbb{X}_{i}} \mid \mathcal{F}_{1}^{T}\right\} .
\end{aligned}
$$


By direct calculations it can be examined that

$$
\begin{aligned}
& E\left(\left\|\Sigma^{\prime}\left[\left(\Sigma \Sigma^{\prime}\right)^{-1}-\left(\Sigma_{\star} \Sigma_{\star}^{\prime}\right)^{-1}\right] \Sigma F_{2}\right\|_{\mathrm{op}}\right) \\
\leq & E\left(\left\|\left(\Sigma \Sigma^{\prime}\right)^{-1}-\left(\Sigma_{\star} \Sigma_{\star}^{\prime}\right)^{-1}\right\|_{\mathrm{op}} \times\left\|\Sigma \Sigma^{\prime}\right\|_{\mathrm{op}} \times\left\|F_{2}\right\|_{\mathrm{op}}\right) \\
\leq & E\left(\left\|\left(\Sigma \Sigma^{\prime}\right)^{-1}-\left(\Sigma_{\star} \Sigma_{\star}^{\prime}\right)^{-1}\right\|_{\mathrm{op}}^{1+\omega}\right)^{1 /(1+\omega)} \\
& \times E\left(\left\|\Sigma \Sigma^{\prime}\right\|_{\mathrm{op}}^{2(1+\omega) / \omega}\right)^{\omega /(2(1+\omega))} E\left(\left\|F_{2}\right\|_{\mathrm{op}}^{2(1+\omega) / \omega}\right)^{\omega /(2(1+\omega))} \\
= & O\left(\left(T^{3} N\right)^{-1 / 2}+(T N)^{-1}\right) T^{3 / 2}=O\left(N^{-1 / 2}+T^{1 / 2} / N\right),
\end{aligned}
$$

and

$$
\begin{aligned}
& E\left(\left\|\Sigma_{\star}^{\prime}\left(\Sigma_{\star} \Sigma_{\star}^{\prime}\right)^{-1} \widetilde{\Sigma} F_{2}\right\|_{\mathrm{op}}\right) \\
\leq & E\left(\left\|\left(\Sigma_{\star} \Sigma_{\star}^{\prime}\right)^{-1}\right\|_{\mathrm{op}}^{1 / 2} \operatorname{Tr}\left(F_{2}^{\prime} \widetilde{\Sigma} \widetilde{\Sigma}^{\prime} F_{2}\right)^{1 / 2}\right) \\
\leq & E\left(\left\|\left(\Sigma_{\star} \Sigma_{\star}^{\prime}\right)^{-1}\right\|_{\mathrm{op}}\right)^{1 / 2} E\left(\operatorname{Tr}\left(F_{2}^{\prime} \widetilde{\Sigma}^{\prime} \widetilde{\Sigma} F_{2}\right)\right)^{1 / 2} \\
= & E\left(\left\|\left(\Sigma_{\star} \Sigma_{\star}^{\prime}\right)^{-1}\right\|_{\mathrm{op}}\right)^{1 / 2} E\left(\operatorname{Tr}\left(F_{2}^{\prime} F_{2}\right)\right)^{1 / 2} O\left(N^{-1 / 2}\right) \\
= & E\left(\left\|\left(\Sigma_{\star} \Sigma_{\star}^{\prime}\right)^{-1}\right\|_{\mathrm{op}}\right)^{1 / 2} E\left(\left\|F_{2}^{\prime} F_{2}\right\|_{\mathrm{op}}\right)^{1 / 2} O\left(N^{-1 / 2}\right)=O\left(N^{-1 / 2}\right) .
\end{aligned}
$$

For any $g \in \mathcal{H}$ with $\|g\|=1$ (implying $|g(x)| \leq c_{\varphi} h^{-1 / 2}$ for any $x$ ), we have $\left\|E\left\{F_{2}^{\prime} \Sigma_{\star}^{\prime}\left(\Sigma_{\star} \Sigma_{\star}^{\prime}\right)^{-1} \widetilde{\Sigma} \tau_{i} g \mid \mathcal{F}_{1}^{T}\right\}\right\|_{2} \leq\left\|F_{2}^{\prime} \Sigma_{\star}^{\prime}\left(\Sigma_{\star} \Sigma_{\star}^{\prime}\right)^{-1}\right\|_{\mathrm{op}}\left\|E\left\{\widetilde{\Sigma} \tau_{i} g \mid \mathcal{F}_{1}^{T}\right\}\right\|_{2}=O_{P}\left(\left\|E\left\{\widetilde{\Sigma} \tau_{i} g \mid \mathcal{F}_{1}^{T}\right\}\right\|_{2}\right)$.

On the other hand, by direct examinations we have

$$
\left\|\widetilde{\Sigma} \tau_{i} g\right\|_{2}^{2}=\sum_{t, l=1}^{T} \bar{v}_{t}^{\prime} \bar{v}_{l} g\left(x_{i t}\right) g\left(x_{i l}\right) .
$$

Meanwhile, for any $t \neq l, \bar{v}_{t}^{\prime} g\left(x_{i t}\right)$ and $\bar{v}_{l} g\left(x_{i l}\right)$ are independent conditional on $\mathcal{F}_{1}^{T}$, and

$$
E\left\{\bar{v}_{l} g\left(x_{i l}\right) \mid \mathcal{F}_{1}^{T}\right\}=\frac{1}{N} E\left\{v_{i l} g\left(x_{i l}\right) \mid \mathcal{F}_{1}^{T}\right\}+\frac{1}{N} \sum_{k \neq i} E\left\{v_{k l} g\left(x_{i l}\right) \mid \mathcal{F}_{1}^{T}\right\}=\frac{1}{N} E\left\{v_{i l} g\left(x_{i l}\right) \mid \mathcal{F}_{1}^{T}\right\} .
$$

The last equality holds because $v_{k l}$ and $g\left(x_{i l}\right)$ are conditional independent (on $\mathcal{F}_{1}^{T}$ ) for $k \neq i$ and the former has mean zero. This leads us to that

$$
\begin{aligned}
E\left\{\left\|\widetilde{\Sigma} \tau_{i} g\right\|_{2}^{2} \mid \mathcal{F}_{1}^{T}\right\} & =\sum_{t=1}^{T} E\left\{\bar{v}_{t}^{\prime} \bar{v}_{t} g\left(x_{i t}\right)^{2} \mid \mathcal{F}_{1}^{T}\right\}+\sum_{t \neq l} E\left\{\bar{v}_{t}^{\prime} \bar{v}_{l} g\left(x_{i t}\right) g\left(x_{i l}\right) \mid \mathcal{F}_{1}^{T}\right\} \\
& =\sum_{t=1}^{T} E\left\{\bar{v}_{t}^{\prime} \bar{v}_{t} g\left(x_{i t}\right)^{2} \mid \mathcal{F}_{1}^{T}\right\}+\sum_{t \neq l} E\left\{\bar{v}_{t}^{\prime} g\left(x_{i t}\right) \mid \mathcal{F}_{1}^{T}\right\} E\left\{\bar{v}_{l} g\left(x_{i l}\right) \mid \mathcal{F}_{1}^{T}\right\} \\
& =\sum_{t=1}^{T} E\left\{\bar{v}_{t}^{\prime} \bar{v}_{t} g\left(x_{i t}\right)^{2} \mid \mathcal{F}_{1}^{T}\right\}+\frac{1}{N^{2}} \sum_{t \neq l} E\left\{v_{i t}^{\prime} g\left(x_{i t}\right) \mid \mathcal{F}_{1}^{T}\right\} E\left\{v_{i l} g\left(x_{i l}\right) \mid \mathcal{F}_{1}^{T}\right\} \\
& =O_{P}\left(\frac{T}{N h}+\frac{T^{2}}{N^{2} h}\right) .
\end{aligned}
$$


Therefore,

$$
\left\|E\left\{F_{2}^{\prime} \Sigma_{\star}^{\prime}\left(\Sigma_{\star} \Sigma_{\star}^{\prime}\right)^{-1} \widetilde{\Sigma} \tau_{i} g \mid \mathcal{F}_{1}^{T}\right\}\right\|_{2}=O_{P}\left(\sqrt{\frac{T}{N h}}+\frac{T}{N \sqrt{h}}\right),
$$

where the $O_{P}$ term is free of $g$.

Similarly, we can show that

$$
\begin{aligned}
& E\left(\left\|\widetilde{\Sigma}^{\prime}\left(\Sigma_{\star} \Sigma_{\star}^{\prime}\right)^{-1} \widetilde{\Sigma} F_{2}\right\|_{\mathrm{op}}\right) \\
\leq & E\left(\left\|\widetilde{\Sigma} \widetilde{\Sigma}^{\prime}\right\|_{\mathrm{op}}^{2}\right)^{1 / 4} E\left(\left\|\left(\Sigma_{\star} \Sigma_{\star}^{\prime}\right)^{-1}\right\|_{\mathrm{op}}^{4}\right)^{1 / 4} E\left(F_{2}^{\prime} \widetilde{\Sigma^{\prime}} \widetilde{\Sigma} F_{2}\right)^{1 / 2} \\
= & O(\sqrt{T / N}) O(1 / T) O(\sqrt{T / N})=O(1 / N) .
\end{aligned}
$$

Combining the above, we get that

$$
\left\|E\left\{\gamma_{2 i}^{\prime} F_{2}^{\prime}\left(P-P_{\star}\right) K_{\mathbb{X}_{i}} \mid \mathcal{F}_{1}^{T}\right\}\right\|=O_{P}\left(\sqrt{\frac{T}{N h}}+\frac{T}{N \sqrt{h}}\right),
$$

where the $O_{P}$ is free of $i \in[N]$. Proof completed.

Lemma S.3. Suppose that Assumptions A2, A4 and A5 hold. Let $\psi$ satisfy the conditions in Lemma A.4. Then

$$
\sup _{\|g\|_{\sup } \leq 1} \frac{1}{\sqrt{N}}\left\|\sum_{i=1}^{N} \psi\left(\mathbb{X}_{i}, g\right)^{\prime}\left(P-P_{\star}\right) K_{\mathbb{X}_{i}}\right\|=O_{P}(1)
$$

and

$$
\sup _{\|g\|_{\mathrm{sup}} \leq 1} \frac{1}{\sqrt{N}}\left\|\sum_{i=1}^{N} E\left(\psi\left(\mathbb{X}_{i}, g\right)^{\prime}\left(P-P_{\star}\right) K_{\mathbb{X}_{i}} \mid \mathcal{F}_{1}^{T}\right)\right\|=O_{P}(1) .
$$

Proof of Lemma S.3. For any $g, \widetilde{g}$ satisfying $\|g\|_{\text {sup }} \leq 1$ and $\|\widetilde{g}\| \leq 1$, the former implies that $\left\|\psi\left(\mathbb{X}_{i}, g\right)\right\|_{2} \leq L \sqrt{h / T}$ for each $i \in[N]$, and the latter implies that $\|\widetilde{g}\|_{\text {sup }} \leq c_{\varphi} h^{-1 / 2}$, by (A.20) we have

$$
\frac{1}{\sqrt{N}}\left|\sum_{i=1}^{N} \psi\left(\mathbb{X}_{i}, g\right)^{\prime}\left(P-P_{\star}\right) \tau_{i} \widetilde{g}\right| \leq \frac{1}{\sqrt{N}} \sum_{i=1}^{N}\left\|\psi\left(\mathbb{X}_{i}, g\right)\right\|_{2}\left\|\tau_{i} \widetilde{g}\right\|_{2}\left\|P-P_{\star}\right\|_{\mathrm{op}}=O_{P}(1),
$$

and

$$
\frac{1}{\sqrt{N}}\left|\sum_{i=1}^{N} E\left(\psi\left(\mathbb{X}_{i}, g\right)^{\prime}\left(P-P_{\star}\right) \tau_{i} \widetilde{g} \mid \mathcal{F}_{1}^{T}\right)\right| \leq L c_{\varphi} \sqrt{N} E\left(\left\|P-P_{\star}\right\|_{\text {op }} \mid \mathcal{F}_{1}^{T}\right)=O_{P}(1) .
$$

Proof is completed.

Proof of Lemma A.4. It follows by Lemma S.3 that we only need to consider the process $Z_{M}^{\star}(g)=$ $\frac{1}{\sqrt{N}} \sum_{i=1}^{N}\left[\psi\left(\mathbb{X}_{i}, g\right)^{\prime} P_{\star} K_{\mathbb{X}_{i}}-E\left\{\psi\left(\mathbb{X}_{i}, g\right)^{\prime} P_{\star} K_{\mathbb{X}_{i}} \mid \mathcal{F}_{1}^{T}\right\}\right]$ for $g \in \mathcal{H}$ where the items in summation are 
independent conditional on $\mathcal{F}_{1}^{T}$. Let $\mathbf{K}_{i}=\left[K\left(X_{i t}, X_{i l}\right)\right]_{1 \leq t, l \leq T}$, a $T \times T$ matrix. By Assumption A4 it follows that $\mathbf{K}_{i} \leq c_{\varphi}^{2} h^{-1} T I_{T}$. For any $g_{1}, g_{2} \in \mathcal{H}$

$$
\begin{aligned}
& \left\|\left(\psi\left(\mathbb{X}_{i}, g_{1}\right)-\psi\left(\mathbb{X}_{i}, g_{2}\right)\right)^{\prime} P_{\star} K_{\mathbb{X}_{i}}\right\|^{2} \\
= & \left(\psi\left(\mathbb{X}_{i}, g_{1}\right)-\psi\left(\mathbb{X}_{i}, g_{2}\right)\right)^{\prime} P_{\star} \mathbf{K}_{i} P_{\star}\left(\psi\left(\mathbb{X}_{i}, g_{1}\right)-\psi\left(\mathbb{X}_{i}, g_{2}\right)\right) \\
\leq & \left(L c_{\varphi}\left\|P_{\star}\right\|_{\text {op }}\left\|g_{1}-g_{2}\right\|_{\text {sup }}\right)^{2}=\left(L c_{\varphi}\left\|g_{1}-g_{2}\right\|_{\text {sup }}\right)^{2} .
\end{aligned}
$$

The last equation follows by $\left\|P_{\star}\right\|_{\mathrm{op}}=1$ since $P_{\star}$ is idempotent. Notice that $\left\{X_{i t}: i \in[N], t \in[T]\right\}$ are conditional independent given $\mathcal{F}_{1}^{T}$. It follows by Pinelis (1994, Theorem 3.5) that for any $r \geq 0$,

$$
P\left(\left\|Z_{M}^{\star}\left(g_{1}\right)-Z_{M}^{\star}\left(g_{2}\right)\right\| \geq r \mid \mathcal{F}_{1}^{T}\right) \leq 2 \exp \left(-\frac{r^{2}}{8 L^{2} c_{\varphi}^{2}\left\|g_{1}-g_{2}\right\|_{\text {sup }}^{2}}\right) .
$$

It follows by Kosorok (2008, Lemma 8.1) that

$$
\|\| Z_{M}^{\star}\left(g_{1}\right)-Z_{M}^{\star}\left(g_{2}\right)\|\|_{\mathcal{F}_{1}^{T}, \psi_{2}} \leq 5 L c_{\varphi}\left\|g_{1}-g_{2}\right\|_{\text {sup }},
$$

where $\|\cdot\|_{\mathcal{F}_{1}^{T}, \psi_{2}}$ denotes the Orlicz-norm conditional on $\mathcal{F}_{1}^{T}$ with respect to $\psi_{2}(s)=\exp \left(s^{2}\right)-1$. This in turn leads to, by Kosorok (2008, Theorem 8.4), that for any $\delta>0$,

$$
\begin{aligned}
& \left\|\sup _{\substack{g_{1}, g_{2} \in \mathcal{G}(p) \\
\left\|g_{1}-g_{2}\right\|_{\text {sup }} \leq \delta}}\right\| Z_{M}^{\star}\left(g_{1}\right)-Z_{M}^{\star}\left(g_{2}\right)\|\|_{\mathcal{F}_{1}^{T}, \psi_{2}} \\
\leq & C\left[\int_{0}^{\delta} \psi_{2}^{-1}\left(D\left(\varepsilon, \mathcal{G}(p),\|\cdot\|_{\text {sup }}\right)\right) d \varepsilon+\delta \psi_{2}^{-1}\left(D\left(\delta, \mathcal{G}(p),\|\cdot\|_{\text {sup }}\right)^{2}\right)\right] \\
= & C J(p, \delta),
\end{aligned}
$$

where $C>0$ is a constant depending on $L, c_{\varphi}$ only. Then we have

$$
\left\|\sup _{\substack{g \in \mathcal{G}(p) \\\|g\|_{\text {sup }} \leq \delta}}\right\| Z_{M}^{\star}(g)\|\|_{\mathcal{F}_{1}^{T}, \psi_{2}} \leq C J(p, \delta) .
$$

It follows again from Kosorok (2008, Lemma 8.1) that for all $\delta>0, r>0$,

$$
P\left(\sup _{\substack{g \in \mathcal{G}(p) \\\|g\|_{\text {sup }} \leq \delta}}\left\|Z_{M}^{\star}(g)\right\| \geq r \mid \mathcal{F}_{1}^{T}\right) \leq 2 \exp \left(-\frac{r^{2}}{C^{2} J(p, \delta)^{2}}\right) .
$$

Let $Q_{N}=\log \left(N^{1 / 2} J(p, 1)\right)-1$. It follows from the proof of $(\mathrm{S} .7)$ that

$$
\begin{aligned}
& P\left(\sup _{g \in \mathcal{G}(p)} \frac{\sqrt{N}\left\|Z_{M}^{\star}(g)\right\|}{\sqrt{N} J\left(p,\|g\|_{\text {sup }}\right)+1} \geq C \sqrt{18 \log \left(Q_{N}\right)} \mid \mathcal{F}_{1}^{T}\right) \\
\leq & 2\left(Q_{N}+2\right) \exp \left(-\frac{18 C^{2} \log \left(Q_{N}\right)}{C^{2} \exp (2)}\right) \leq \frac{2\left(Q_{N}+2\right)}{Q_{N}^{2}} .
\end{aligned}
$$


Taking expectation on both sides of (S.12), we get that

$$
P\left(\sup _{g \in \mathcal{G}(p)} \frac{\sqrt{N}\left\|Z_{M}^{\star}(g)\right\|}{\sqrt{N} J\left(p,\|g\|_{\text {sup }}\right)+1} \geq C \sqrt{18 \log \left(Q_{N}\right)}\right)=o(1), \text { as } N \rightarrow \infty .
$$

This shows that, with probability approaching one,

$$
\sup _{g \in \mathcal{G}(p)} \frac{\sqrt{N}\left\|Z_{M}^{\star}(g)\right\|}{\sqrt{N} J\left(p,\|g\|_{\text {sup }}\right)+1} \leq C \sqrt{18 \log \left(Q_{N}\right)} .
$$

Since $\|g\|_{\text {sup }} \leq 1$ for any $g \in \mathcal{G}$ and $J(p, \delta)$ is increasing in $\delta$, the above inequality implies that, with probability approaching one,

$$
\sup _{g \in \mathcal{G}(p)}\left\|Z_{M}^{\star}(g)\right\| \leq C \sqrt{18 \log \left(Q_{N}\right)}\left(J(p, 1)+N^{-1 / 2}\right) .
$$

Combining with Lemma S.3, we get that

$$
\begin{aligned}
\sup _{g \in \mathcal{G}(p)}\left\|Z_{M}(g)\right\| & \leq \sup _{g \in \mathcal{G}(p)}\left\|Z_{M}(g)-Z_{M}^{\star}(g)\right\|+\sup _{g \in \mathcal{G}(p)}\left\|Z_{M}^{\star}(g)\right\| \\
& =O_{P}\left(1+\sqrt{\log \log (N J(p, 1))}\left(J(p, 1)+N^{-1 / 2}\right)\right) .
\end{aligned}
$$

Proof completed.

Proof of Lemma A.5. By (2.5), we have $e_{i}^{\prime}=\epsilon_{i}^{\prime}-\Delta_{i}, \bar{v}=\epsilon_{i}^{\prime}-\Delta_{i}\left(\bar{X}-\bar{\Gamma}_{1}^{\prime} F_{1}^{\prime}-\bar{\Gamma}_{2}^{\prime} F_{2}^{\prime}\right)$ and

$$
\begin{aligned}
\left(Y_{i}-\tau_{i} g_{\eta}\right)^{\prime} P K_{\mathbb{X}_{i}} & =\left[\tau_{i}\left(g_{0}-g_{\eta}\right)+\Sigma^{\prime} \beta_{i}+e_{i}\right]^{\prime} P K_{\mathbb{X}_{i}} \\
& =\left[\tau_{i}\left(g_{0}-g_{\eta}\right)+e_{i}\right]^{\prime} P K_{\mathbb{X}_{i}} \\
& =\left[\tau_{i}\left(g_{0}-g_{\eta}\right)+\epsilon_{i}+F_{2} \bar{\Gamma}_{2} \Delta_{i}^{\prime}\right]^{\prime} P K_{\mathbb{X}_{i}} .
\end{aligned}
$$

By the definition of $g_{\eta}$ in the proof Theorem 4.1 and (S.13), we get that

$$
S_{M, \eta}\left(g_{\eta}\right)=S_{M, \eta}\left(g_{\eta}\right)-S_{M, \eta}^{\star}\left(g_{\eta}\right)=T_{1}+T_{2}-T_{3}+W_{\eta} g_{\eta}-E\left(W_{\eta} g_{\eta} \mid \mathcal{F}_{1}^{T}\right)=T_{1}+T_{2}-T_{3}
$$

where

$$
\begin{aligned}
& T_{1}=\frac{1}{N T} \sum_{i=1}^{N}\left[\epsilon_{i}^{\prime} P K_{\mathbb{X}_{i}}-E\left(\epsilon_{i}^{\prime} P K_{\mathbb{X}_{i}} \mid \mathcal{F}_{1}^{T}\right)\right], \\
& T_{2}=\frac{1}{N T} \sum_{i=1}^{N}\left[\Delta_{i} F_{2}^{\prime} P K_{\mathbb{X}_{i}}-E\left(\Delta_{i} F_{2}^{\prime} P K_{\mathbb{X}_{i}} \mid \mathcal{F}_{1}^{T}\right)\right], \\
& T_{3}=\kappa\left(g_{\eta}-g_{0}\right) .
\end{aligned}
$$

Recall that $\kappa$ is defined in the proof of Theorem 4.1. It is worthwhile to mention that the terms $W_{\eta} g_{\eta}$ and $E\left(W_{\eta} g_{\eta} \mid \mathcal{F}_{1}^{T}\right)$ cancel each other in (S.14) thanks to $W_{\eta} g_{\eta} \in \mathcal{F}_{1}^{T}$. Next, we will bound $T_{1}, T_{2}, T_{3}$ respectively. 
First of all, by (A.23) and (A.24), it yields that

$$
\left\|T_{3}\right\|=o_{P}(1)\left\|g_{\eta}-g_{0}\right\|=o_{P}\left(\frac{1}{\sqrt{N T h}}+\frac{1}{N \sqrt{h}}+\sqrt{\eta}\right) .
$$

Secondly, the independence of $\epsilon_{i}$ and $\mathbb{X}_{i}, F_{1}, F_{2}$ tells us that

$$
T_{1}=\frac{1}{N T} \sum_{i=1}^{N} \epsilon_{i}^{\prime} P K_{\mathbb{X}_{i}}
$$

Again by the independence assumption and direct calculations, we have

$$
\begin{aligned}
E\left(\left\|T_{1}\right\|^{2} \mid \mathcal{F}_{1}^{T}\right) & =\frac{1}{N^{2} T^{2}} \sum_{i=1}^{N} E\left(\epsilon_{i}^{\prime} P<K_{\mathbb{X}_{i}}, K_{\mathbb{X}_{i}}>P^{\prime} \epsilon_{i} \mid \mathcal{F}_{1}^{T}\right) \\
& =\frac{1}{N^{2} T^{2}} \sum_{i=1}^{N} E\left(\epsilon_{i}^{\prime} P \mathbf{K}_{i} P^{\prime} \epsilon_{i} \mid \mathcal{F}_{1}^{T}\right) \\
& =\frac{1}{N^{2} T^{2}} \sum_{i=1}^{N} \operatorname{Tr}\left(E\left(P \mathbf{K}_{i} P^{\prime} \epsilon_{i} \epsilon_{i}^{\prime}\right) \mid \mathcal{F}_{1}^{T}\right) \\
& =\frac{\sigma_{\epsilon}^{2}}{N T^{2}} E\left\{\operatorname{Tr}\left(P \mathbf{K}_{i} P^{\prime}\right) \mid \mathcal{F}_{1}^{T}\right\} \\
& \leq \frac{\sigma_{\epsilon}^{2}}{N T^{2}} E\left\{\operatorname{Tr}\left(\mathbf{K}_{i}\right) \mid \mathcal{F}_{1}^{T}\right\} \\
& =O_{P}\left(\frac{1}{N T h}\right),
\end{aligned}
$$

where we are using the facts that $\mathbf{K}_{i}=\left[K\left(X_{i t}, X_{i l}\right)\right]_{1 \leq t, l \leq T}$ and $\operatorname{Tr}\left(\mathbf{K}_{i}\right) \leq T c_{\varphi}^{2} h^{-1}$ derived from (A.19). So it follows

$$
\left\|T_{1}\right\|=O_{P}\left(\frac{1}{\sqrt{N T h}}\right)
$$

Lastly, we will handle $T_{2}$ as follows. Since $F_{2}^{\prime} P_{\star}=0$ (see Section A.3), it follows that

$$
T_{2}=\frac{1}{N T} \sum_{i=1}^{N}\left[\Delta_{i} F_{2}^{\prime}\left(P-P_{\star}\right) K_{\mathbb{X}_{i}}-E\left(\Delta_{i} F_{2}^{\prime} P K_{\mathbb{X}_{i}} \mid \mathcal{F}_{1}^{T}\right)\right] .
$$

By the proof and notation in Lemma A.3, it can be shown that

$$
\begin{aligned}
& P_{\star}-P \\
= & \Sigma^{\prime}\left(\left(\Sigma \Sigma^{\prime}\right)^{-1}-\left(\Sigma_{\star} \Sigma_{\star}^{\prime}\right)^{-1}\right) \Sigma+\Sigma_{\star}^{\prime}\left(\Sigma_{\star} \Sigma_{\star}^{\prime}\right)^{-1} \widetilde{\Sigma}+\widetilde{\Sigma}^{\prime}\left(\Sigma_{\star} \Sigma_{\star}^{\prime}\right)^{-1} \Sigma_{\star}+\widetilde{\Sigma}^{\prime}\left(\Sigma_{\star} \Sigma_{\star}^{\prime}\right)^{-1} \widetilde{\Sigma} .
\end{aligned}
$$


Consequently, $T_{2}$ has following decomposition:

$$
\begin{aligned}
& T_{2} \\
= & \frac{1}{N T} \sum_{i=1}^{N}\left[\Delta_{i} F_{2}^{\prime}\left(\Sigma^{\prime}\left(\left(\Sigma \Sigma^{\prime}\right)^{-1}-\left(\Sigma_{\star} \Sigma_{\star}^{\prime}\right)^{-1}\right) \Sigma\right) K_{\mathbb{X}_{i}}-E\left(\Delta_{i} F_{2}^{\prime}\left(\Sigma^{\prime}\left(\left(\Sigma \Sigma^{\prime}\right)^{-1}-\left(\Sigma_{\star} \Sigma_{\star}^{\prime}\right)^{-1}\right) \Sigma\right) K_{\mathbb{X}_{i}} \mid \mathcal{F}_{1}^{T}\right)\right] \\
& +\frac{1}{N T} \sum_{i=1}^{N}\left[\Delta_{i} F_{2}^{\prime} \Sigma_{\star}^{\prime}\left(\Sigma_{\star} \Sigma_{\star}^{\prime}\right)^{-1} \widetilde{\Sigma} K_{\mathbb{X}_{i}}-E\left(\Delta_{i} F_{2}^{\prime} \Sigma_{\star}^{\prime}\left(\Sigma_{\star} \Sigma_{\star}^{\prime}\right)^{-1} \widetilde{\Sigma} K_{\mathbb{X}_{i}} \mid \mathcal{F}_{1}^{T}\right)\right] \\
& +\frac{1}{N T} \sum_{i=1}^{N}\left[\Delta_{i} F_{2}^{\prime} \widetilde{\Sigma}^{\prime}\left(\Sigma_{\star} \Sigma_{\star}^{\prime}\right)^{-1} \Sigma_{\star} K_{\mathbb{X}_{i}}-E\left(\Delta_{i} F_{2}^{\prime} \widetilde{\Sigma}^{\prime}\left(\Sigma_{\star} \Sigma_{\star}^{\prime}\right)^{-1} \Sigma_{\star} K_{\mathbb{X}_{i}} \mid \mathcal{F}_{1}^{T}\right)\right] \\
& +\frac{1}{N T} \sum_{i=1}^{N}\left[\Delta_{i} F_{2}^{\prime} \widetilde{\Sigma}^{\prime}\left(\Sigma_{\star} \Sigma_{\star}^{\prime}\right)^{-1} \widetilde{\Sigma} K_{\mathbb{X}_{i}}-E\left(\Delta_{i} F_{2}^{\prime} \widetilde{\Sigma}^{\prime}\left(\Sigma_{\star} \Sigma_{\star}^{\prime}\right)^{-1} \widetilde{\Sigma} K_{\mathbb{X}_{i}} \mid \mathcal{F}_{1}^{T}\right)\right] \\
\equiv & T_{21}+T_{22}+T_{23}+T_{24} .
\end{aligned}
$$

The rest of the proof proceeds to bound the terms $T_{2 i}, i=1,2,3,4$. By (S.9) in the proof of Lemma A.3, we obtain the following:

$$
\begin{aligned}
E\left(\left\|F_{2}^{\prime} \Sigma^{\prime}\left(\left(\Sigma \Sigma^{\prime}\right)^{-1}-\left(\Sigma_{\star} \Sigma_{\star}^{\prime}\right)^{-1}\right) \Sigma\right\|_{\mathrm{op}}\right) & =O\left(N^{-1 / 2}+T^{1 / 2} / N\right), \\
E\left(\left\|F_{2}^{\prime} \widetilde{\Sigma}^{\prime}\left(\Sigma_{\star} \Sigma_{\star}^{\prime}\right)^{-1} \Sigma_{\star}\right\|_{\mathrm{op}}\right) & =O\left(N^{-1 / 2}\right), \\
E\left(\left\|F_{2}^{\prime} \widetilde{\Sigma}^{\prime}\left(\Sigma_{\star} \Sigma_{\star}^{\prime}\right)^{-1} \widetilde{\Sigma}\right\|_{\mathrm{op}}\right) & =O(1 / N) .
\end{aligned}
$$

Therefore, it follows that

$$
\begin{aligned}
& \left.\| E\left(\frac{1}{N T} \sum_{i=1}^{N} \Delta_{i} F_{2}^{\prime} \Sigma^{\prime}\left(\left(\Sigma \Sigma^{\prime}\right)^{-1}-\left(\Sigma_{\star} \Sigma_{\star}^{\prime}\right)^{-1}\right)\right) \Sigma K_{\mathbb{X}_{i}} \mid \mathcal{F}_{1}^{T}\right) \| \\
\leq & \left.\frac{1}{N T} \sum_{i=1}^{N} E\left(\| \Delta_{i} F_{2}^{\prime} \Sigma^{\prime}\left(\left(\Sigma \Sigma^{\prime}\right)^{-1}-\left(\Sigma_{\star} \Sigma_{\star}^{\prime}\right)^{-1}\right)\right) \Sigma K_{\mathbb{X}_{i}} \| \mid \mathcal{F}_{1}^{T}\right) \\
\leq & \left.\frac{1}{N T} \sum_{i=1}^{N}\left\|\Delta_{i}\right\|_{2} E\left(\| F_{2}^{\prime} \Sigma^{\prime}\left(\left(\Sigma \Sigma^{\prime}\right)^{-1}-\left(\Sigma_{\star} \Sigma_{\star}^{\prime}\right)^{-1}\right)\right) \Sigma \|_{\mathrm{op}} \sqrt{\sum_{t=1}^{T}\left\|K_{X_{i t}}\right\|^{2} \mid \mathcal{F}_{1}^{T}}\right) \\
\leq & \left.\frac{1}{N T} \sum_{i=1}^{N}\left\|\Delta_{i}\right\|_{2} E\left(\| F_{2}^{\prime} \Sigma^{\prime}\left(\left(\Sigma \Sigma^{\prime}\right)^{-1}-\left(\Sigma_{\star} \Sigma_{\star}^{\prime}\right)^{-1}\right)\right) \Sigma \|_{\mathrm{op}} \sqrt{T c_{\varphi}^{2} h^{-1}} \mid \mathcal{F}_{1}^{T}\right) \\
\leq & \frac{1}{\sqrt{T}} \sup _{1 \leq i \leq N}\left\|\Delta_{i}\right\|_{2} \sqrt{c_{\varphi}^{2} h^{-1}} E\left(\left\|F^{\prime} \Sigma^{\prime}\left(\left(\Sigma \Sigma^{\prime}\right)^{-1}-\left(\Sigma_{\star} \Sigma_{\star}^{\prime}\right)^{-1}\right) \Sigma\right\|_{\mathrm{op}} \mid \mathcal{F}_{1}^{T}\right) \\
= & O_{P}\left(\frac{1}{\sqrt{N T h}}+\frac{1}{N \sqrt{h}}\right) .
\end{aligned}
$$


As a consequence, $\left\|T_{21}\right\|=O_{P}\left((N T h)^{-1 / 2}+N^{-1} h^{-1 / 2}\right)$. Similarly,

$$
\begin{aligned}
& E\left\{\left\|E\left(\frac{1}{N T} \sum_{i=1}^{N} \Delta_{i} F_{2}^{\prime} \widetilde{\Sigma}^{\prime}\left(\Sigma_{\star} \Sigma_{\star}^{\prime}\right)^{-1} \Sigma_{\star} K_{\mathbb{X}_{i}} \mid \mathcal{F}_{1}^{T}\right)\right\|\right\}=O_{P}\left(\frac{1}{\sqrt{N T h}}\right), \\
& E\left\{\left\|E\left(\frac{1}{N T} \sum_{i=1}^{N} \Delta_{i} F_{2}^{\prime} \widetilde{\Sigma}^{\prime}\left(\Sigma_{\star} \Sigma_{\star}^{\prime}\right)^{-1} \widetilde{\Sigma} K_{\mathbb{X}_{i}} \mid \mathcal{F}_{1}^{T}\right)\right\|\right\}=O_{P}\left(\frac{1}{N \sqrt{T h}}\right) .
\end{aligned}
$$

So it follows that $\left\|T_{23}\right\|=O_{P}\left((N T h)^{-1 / 2}\right)$ and $\left\|T_{24}\right\|=O_{P}\left(N^{-1}(T h)^{-1 / 2}\right)$. Finally, we will handle $T_{22}$. Let $W=F_{2}^{\prime} \Sigma_{\star}^{\prime}\left(\Sigma_{\star} \Sigma_{\star}^{\prime}\right)^{-1}$. It can be easily seen from (S.10) that $W \in \mathcal{F}_{1}^{T}$ and $\|W\|_{\text {op }}=O_{P}(1)$. To bound $T_{22}$, notice

$$
\widetilde{\Sigma} K_{\mathbb{X}_{i}}=\left(\begin{array}{c}
0_{q_{1} \times T} \\
\sum_{t=1}^{T} \bar{v}_{t} K_{X_{i t}}
\end{array}\right)=\left(\begin{array}{c}
0_{q_{1} \times T} \\
\sum_{t=1}^{T} \bar{v}_{t 1} K_{X_{i t}} \\
\sum_{t=1}^{T} \bar{v}_{t 2} K_{X_{i t}} \\
\cdots \\
\cdots \\
\sum_{t=1}^{T} \bar{v}_{t d} K_{X_{i t}}
\end{array}\right),
$$

where $\bar{v}_{t i}$ is the $i$ th element of vector $\bar{v}_{t}$. By direct calculations, it follows that

$$
\begin{aligned}
\left\|T_{22}\right\| & =\left\|\frac{1}{N T} \sum_{i=1}^{N}\left\{\Delta_{i} W \widetilde{\Sigma} K_{\mathbb{X}_{i}}-E\left(\Delta_{i} W \widetilde{\Sigma} K_{\mathbb{X}_{i}} \mid \mathcal{F}_{1}^{T}\right)\right\}\right\| \\
& \leq \frac{1}{N T} \sum_{i=1}^{N}\left\|\Delta_{i} W \widetilde{\Sigma} K_{\mathbb{X}_{i}}-E\left(\Delta_{i} W \widetilde{\Sigma} K_{\mathbb{X}_{i}} \mid \mathcal{F}_{1}^{T}\right)\right\| \\
& \leq \frac{1}{N T} \sum_{i=1}^{N}\left\|\Delta_{i}\right\|_{2}\|W\|_{\mathrm{op}} \sqrt{\sum_{l=1}^{d}\left\|\sum_{t=1}^{T}\left(\bar{v}_{t l} K_{X_{i t}}-E\left(\bar{v}_{t l} K_{X_{i t}} \mid \mathcal{F}_{1}^{T}\right)\right)\right\|^{2} .}
\end{aligned}
$$

By (S.17), it suffices to find the rate of

$$
\frac{1}{N T} \sum_{i=1}^{N} \sqrt{\sum_{l=1}^{d}\left\|\sum_{t=1}^{T}\left(\bar{v}_{t l} K_{X_{i t}}-E\left(\bar{v}_{t l} K_{X_{i t}} \mid \mathcal{F}_{1}^{T}\right)\right)\right\|^{2}}
$$

Because $d$ is finite and fixed, to simplify our technical arguments, assume $d=1$ without loss of generality. Direct examinations give the following decomposition:

$$
\begin{aligned}
& \left\|\sum_{t=1}^{T}\left(\bar{v}_{t l} K_{X_{i t}}-E\left(\bar{v}_{t l} K_{X_{i t}} \mid \mathcal{F}_{1}^{T}\right)\right)\right\|^{2} \\
= & \frac{1}{N^{2}}\left\|\sum_{t=1}^{T} \sum_{j=1}^{N}\left(v_{j t l} K_{X_{i t}}-E\left(v_{j t l} K_{X_{i t}} \mid \mathcal{F}_{1}^{T}\right)\right)\right\|^{2} \\
= & \frac{2}{N^{2}}\left\|\sum_{t=1}^{T} \sum_{j \neq i}^{N}\left(v_{j t l} K_{X_{i t}}-E\left(v_{j t l} K_{X_{i t}} \mid \mathcal{F}_{1}^{T}\right)\right)\right\|^{2}+\frac{2}{N^{2}}\left\|\sum_{t=1}^{T}\left(v_{i t l} K_{X_{i t}}-E\left(v_{i t l} K_{X_{i t}} \mid \mathcal{F}_{1}^{T}\right)\right)\right\|^{2} \\
\equiv & T_{221}+T_{222} .
\end{aligned}
$$


When $i \neq j, v_{j t l}$ is independent of $X_{i t}, \mathcal{F}_{1}^{T}$, so it follows that

$$
\begin{aligned}
& E\left\{\left\|\sum_{t=1}^{T} \sum_{j \neq i}\left(v_{j t l} K_{X_{i t}}-E\left(v_{j t l} K_{X_{i t}} \mid \mathcal{F}_{1}^{T}\right)\right)\right\|^{2} \mid \mathcal{F}_{1}^{T}\right\} \\
= & E\left\{\left\|\sum_{t=1}^{T} \sum_{j \neq i} v_{j t l} K_{X_{i t}}\right\|^{2} \mid \mathcal{F}_{1}^{T}\right\} \\
= & E\left\{\sum_{t, t^{\prime}=1}^{T} \sum_{j, j^{\prime} \neq i} v_{j t l} v_{j^{\prime} t^{\prime} l} K\left(X_{i t}, X_{i t^{\prime}}\right) \mid \mathcal{F}_{1}^{T}\right\} \\
= & \left.\sum_{t=1}^{T} \sum_{j \neq i} E\left(v_{j t l}^{2}\right) E\left(K\left(X_{i t}, X_{i t}\right)\right\} \mid \mathcal{F}_{1}^{T}\right) \\
\leq & N T E\left(v_{11 l}^{2}\right) c_{\varphi}^{2} h^{-1},
\end{aligned}
$$

As a consequence, $T_{221}=O_{P}\left(T(N h)^{-1}\right)$. To deal with $T_{222}$, by Cauchy inequality, it yields that

$$
\begin{aligned}
E\left\{\left\|\sum_{t=1}^{T}\left(v_{i t l} K_{X_{i t}}\right)\right\|^{2} \mid \mathcal{F}_{1}^{T}\right\} & \leq E\left\{\sum_{t=1}^{T} v_{i t l}^{2} \sum_{t=1}^{T}\left\|K_{X_{i t}}\right\|^{2} \mid \mathcal{F}_{1}^{T}\right\} \\
& \leq E\left(\sum_{t=1}^{T} v_{i t l}^{2}\right) T c_{\varphi}^{2} h^{-1} \\
& =E\left(v_{11 l}^{2}\right) T^{2} c_{\varphi} h^{-1},
\end{aligned}
$$

which further implies $T_{222}=O_{P}\left(T^{2}\left(N^{2} h\right)^{-1}\right)$. By Jensen's inequality and $d=1$, it follows that

$$
\begin{aligned}
(S .18) & =E\left(\frac{1}{N T} \sum_{i=1}^{N} \sqrt{\left.\left\|\sum_{t=1}^{T}\left(\bar{v}_{t l} K_{X_{i t}}-E\left(\bar{v}_{t l} K_{X_{i t}} \mid \mathcal{F}_{1}^{T}\right)\right)\right\|^{2} \mid \mathcal{F}_{1}^{T}\right)}\right. \\
& \leq \frac{1}{N T} \sum_{i=1}^{N} \sqrt{E\left(\left\|\sum_{t=1}^{T}\left(\bar{v}_{t l} K_{X_{i t}}-E\left(\bar{v}_{t l} K_{X_{i t}} \mid \mathcal{F}_{1}^{T}\right)\right)\right\|^{2} \mid \mathcal{F}_{1}^{T}\right)} \\
& \leq \sqrt{2 c_{\varphi}^{2} E\left(v_{11 l}^{2}\right)\left(\frac{1}{N T h}+\frac{1}{N^{2} h}\right)} \\
& =O_{P}\left(\frac{1}{\sqrt{N T h}}+\frac{1}{N \sqrt{h}}\right) .
\end{aligned}
$$

Combining (S.17) and (S.19), it follows that $\left\|T_{22}\right\|=O_{P}\left((N T h)^{-1 / 2}+\left(N h^{1 / 2}\right)^{-1}\right)$. As a consequence, we have

$$
\left\|T_{2}\right\|=O_{P}\left(\frac{1}{\sqrt{N T h}}+\frac{1}{N \sqrt{h}}\right) .
$$

Combining (S.15), (S.16) and (S.20), it yields that

$$
\left\|S_{M, \eta}\left(g_{\eta}\right)\right\|=O_{P}\left(\frac{1}{\sqrt{N T h}}+\frac{1}{N \sqrt{h}}\right)+o_{P}(\sqrt{\eta}) .
$$

Proof completed. 
Next we will prove Lemmas A.6, A.7 and A.8. For this purpose, let us introduce a set of notation. Define $V_{N T \star}, A_{N T \star}, V_{N T m \star}, A_{N T m \star}, H_{N T m \star}$ as follows,

$$
\begin{array}{r}
V_{N T \star}=\frac{1}{N T} \sum_{i=1}^{N} K_{\mathbb{X}_{i}}\left(x_{0}\right)^{\prime} P_{\star} K_{\mathbb{X}_{i}}\left(x_{0}\right), A_{N T \star}=V_{N T \star}^{-1 / 2}, \\
V_{N T m \star}=\frac{1}{N T} \sum_{i=1}^{N} \phi_{m}^{\prime} \Phi_{i}^{\prime} P_{\star} \Phi_{i} \phi_{m}, A_{N T m \star}=V_{N T m \star}^{-1 / 2}, H_{N T m \star}=\frac{1}{N T} \sum_{i=1}^{N} \Phi_{i}^{\prime} P_{\star} \Phi_{i} .
\end{array}
$$

Proof of Lemma A.6. Define

$$
Q_{i \star}=E\left(\frac{\Phi_{i}^{\prime} P_{\star} \Phi_{i}}{T} \mid \mathcal{F}_{1}^{T}\right), \bar{Q}_{\star}=\frac{1}{N} \sum_{i=1}^{N} Q_{i \star}, Q_{i}=E\left(\frac{\Phi_{i}^{\prime} P \Phi_{i}}{T} \mid \mathcal{F}_{1}^{T}\right), \bar{Q}=\frac{1}{N} \sum_{i=1}^{N} Q_{i}=I_{m} .
$$

Notice that, conditioning on $\mathcal{F}_{1}^{T}, \Phi_{i}$ are independent. Hence, by Chebyshev's inequality, it follows that

$$
\begin{aligned}
P\left(\left\|H_{N T m \star}-\bar{Q}_{\star}\right\|_{F}>\epsilon \mid \mathcal{F}_{1}^{T}\right) & =P\left(\left\|\frac{1}{N} \sum_{i=1}^{N}\left(\frac{\Phi_{i}^{\prime} P_{\star} \Phi_{i}}{T}-Q_{i \star}\right)\right\|_{F}>\epsilon \mid \mathcal{F}_{1}^{T}\right) \\
& \leq \frac{1}{\epsilon^{2} N^{2}} E\left\{\operatorname{Tr}\left(\left[\sum_{i=1}^{N}\left(\frac{\Phi_{i}^{\prime} P_{\star} \Phi_{i}}{T}-Q_{i \star}\right)\right]^{2}\right) \mid \mathcal{F}_{1}^{T}\right\} \\
& =\frac{1}{\epsilon^{2} N^{2}} \sum_{i=1}^{N} \operatorname{Tr}\left\{E\left(\left[\frac{\Phi_{i}^{\prime} P_{\star} \Phi_{i}}{T}-Q_{i \star}\right]^{2}\right) \mid \mathcal{F}_{1}^{T}\right\} \\
& =\frac{1}{\epsilon^{2} N^{2}} \sum_{i=1}^{N} E\left(\left\|\frac{\Phi_{i}^{\prime} P_{\star} \Phi_{i}}{T}-Q_{i \star}\right\|_{F}^{2} \mid \mathcal{F}_{1}^{T}\right) \\
& \leq \frac{1}{\epsilon^{2} N^{2}} \sum_{i=1}^{N} E\left(\left\|\frac{\Phi_{i}^{\prime} P_{\star} \Phi_{i}}{T}\right\|_{F}^{2} \mid \mathcal{F}_{1}^{T}\right) \\
& \leq \frac{1}{\epsilon^{2} N^{2} T^{2}} \sum_{i=1}^{N} E\left(\left\|\Phi_{i}\right\|_{F}^{4} \mid \mathcal{F}_{1}^{T}\right) \\
& \leq \frac{m^{2}\left(c_{\varphi}+1\right)^{4}}{\epsilon^{2} N}
\end{aligned}
$$

As a consequence, it follows that,

$$
P\left(\left\|H_{N T m \star}-\bar{Q}_{\star}\right\|_{F}>\frac{\epsilon m\left(c_{\varphi}+1\right)^{2}}{\sqrt{N}} \mid \mathcal{F}_{1}^{T}\right) \leq \frac{1}{\epsilon^{2}} .
$$

By taking expectation on both sides, we have

$$
P\left(\left\|H_{N T m \star}-\bar{Q}_{\star}\right\|_{F}>\frac{\epsilon m\left(c_{\varphi}+1\right)^{2}}{\sqrt{N}}\right) \leq \frac{1}{\epsilon^{2}} .
$$

Since $c_{\varphi}=O_{P}(1)$, we obtain

$$
\left\|H_{N T m \star}-\bar{Q}_{\star}\right\|_{F}=O_{P}\left(m N^{-1 / 2}\right) .
$$


By Lemma A.3, we have

$$
\begin{aligned}
E\left(\left\|H_{N T m}-H_{N T m \star}\right\|_{F} \mid \mathcal{F}_{1}^{T}\right) & \leq \frac{1}{N T} \sum_{i=1}^{N} E\left(\left\|\Phi_{i}^{\prime}\left(P-P_{\star}\right) \Phi_{i}\right\|_{F} \mid \mathcal{F}_{1}^{T}\right) \\
& \leq \frac{1}{N T} \sum_{i=1}^{N} E\left(\left\|\left(P-P_{\star}\right)\right\|_{\text {op }}\left\|\Phi_{i}\right\|_{F}^{2} \mid \mathcal{F}_{1}^{T}\right) \\
& \leq \frac{1}{N T} \sum_{i=1}^{N} m T c_{\varphi}^{2} E\left(\left\|\left(P-P_{\star}\right)\right\|_{\text {op }} \mid \mathcal{F}_{1}^{T}\right) \\
& =O_{P}\left(\frac{m}{\sqrt{N}}\right) .
\end{aligned}
$$

Again by Lemma A.3 and similar calculations, it follows that

$$
\begin{aligned}
\left\|\bar{Q}-\bar{Q}_{\star}\right\|_{F} & =\left\|E\left(\frac{1}{N T} \sum_{i=1}^{N} \Phi_{i}^{\prime}\left(P-P_{\star}\right) \Phi_{i} \mid \mathcal{F}_{1}^{T}\right)\right\|_{F} \\
& \leq \frac{1}{N T} \sum_{i=1}^{N} E\left(\left\|\Phi_{i}^{\prime}\left(P-P_{\star}\right) \Phi_{i}\right\|_{F} \mid \mathcal{F}_{1}^{T}\right) \\
& =O_{P}\left(\frac{m}{\sqrt{N}}\right) .
\end{aligned}
$$

Combining (S.21), (S.22) and (S.23), it yields that

$$
\left\|H_{N T m}-I_{m}\right\|_{F}=\left\|H_{N T m}-\bar{Q}\right\|_{F}=O_{P}\left(\frac{m}{\sqrt{N}}\right)=o_{P}(1) .
$$

To the end of the proof, we quantify the minimal and maximal eigenvalues of $H_{N T m}$ as follows.

$$
\begin{aligned}
\lambda_{\min }\left(H_{N T m}\right) & =\min _{\|u\|_{2}=1} u^{\prime} H_{N T m} u \\
& \geq \min _{\|u\|_{2}=1} u^{\prime} I_{m} u-\min _{\|u\|_{2}=1}\left|u^{\prime}\left(H_{N T m}-I_{m}\right) u\right| \\
& =1-\left\|H_{N T m}-I_{m}\right\|_{\mathrm{op}} \\
& =1+o_{P}(1),
\end{aligned}
$$

and

$$
\begin{aligned}
\lambda_{\max }\left(H_{N T m}\right) & =\max _{\|u\|_{2}=1} u^{\prime} H_{N T m} u \\
& \leq \max _{\|u\|_{2}=1} u^{\prime} I_{m} u+\max _{\|u\|_{2}=1}\left|u^{\prime}\left(H_{N T m}-I_{m}\right) u\right| \\
& =1+\left\|H_{N T m}-I_{m}\right\|_{\mathrm{op}} \\
& =1+o_{P}(1),
\end{aligned}
$$

where have used the trivial inequality $\left\|H_{N T m}-I_{m}\right\|_{\mathrm{op}} \leq\left\|H_{N T m}-I_{m}\right\|_{F}=o_{P}(1)$. Proof completed. 
Proof of Lemma A.7. By Lemma A.6, we find a lower bound for $V_{N T m}$ and an upper bound for $A_{N T m}$ as follows:

$$
\begin{aligned}
& V_{N T m}=\phi_{m}^{\prime} H_{N T m} \phi_{m} \geq \lambda_{\min }\left(H_{N T m}\right)\left\|\phi_{m}\right\|_{2}^{2} \geq \lambda_{\min }\left(H_{N T m}\right) C \\
& A_{N T m}=V_{N T m}^{-1 / 2} \leq \lambda_{\min }^{-1 / 2}\left(H_{N T m}\right)\left\|\phi_{m}^{\prime}\right\|_{2}^{-1}=O_{P}(1)\left(\sum_{\nu=1}^{m} \frac{\varphi_{\nu}^{2}\left(x_{0}\right)}{\left(1+\eta \rho_{\nu}\right)^{2}}\right)^{-1 / 2}=O_{P}(1) .
\end{aligned}
$$

Define $L_{i}\left(x_{0}\right)=K_{\mathbb{X}_{i}}\left(x_{0}\right)-\Phi_{i} \phi_{m}$. Then it follows that

$$
E\left(\left\|L_{i}\right\|_{2}^{2} \mid \mathcal{F}_{1}^{T}\right) \leq T c_{\varphi}^{4}\left(\sum_{\nu=m+1}^{\infty} \frac{1}{1+\eta \rho_{\nu}}\right)^{2} \equiv T c_{\varphi}^{4} D_{m}^{2} .
$$

Directly calculation shows that

$$
\left|V_{N T}-V_{N T m}\right| \leq\left|\frac{2}{N T} \sum_{i=1}^{N} L_{i}^{\prime} P K_{\mathbb{X}_{i}}\right|+\left|\frac{1}{N T} \sum_{i=1}^{N} L_{i}^{\prime} P L_{i}\right| \equiv 2|T 1|+|T 2| .
$$

Let $R_{x_{0}}(\cdot)=\sum_{\nu=m+1}^{\infty} \frac{\varphi_{\nu}\left(x_{0}\right) \varphi_{\nu}(\cdot)}{1+\eta \rho_{\nu}}$. Notice $L_{i}=\tau_{i} R_{x_{0}}$ and $E\left(T_{1} \mid \mathcal{F}_{1}^{T}\right)=V\left(K_{x_{0}}, R_{x_{0}}\right)$. Similar to the proof of Lemma A.6, we can show that

$$
E\left(\left|T_{1}-V\left(K_{x_{0}}, R_{x_{0}}\right)\right| \mid \mathcal{F}_{1}^{T}\right)=O_{P}\left(\frac{D_{m}}{\sqrt{N} h}\right) .
$$

Meanwhile we have the following

$$
V\left(K_{x_{0}}, R_{x_{0}}\right)=\sum_{\nu=m+1}^{\infty} \frac{\varphi_{\nu}^{2}\left(x_{0}\right)}{\left(1+\eta \rho_{\nu}\right)^{2}} \leq c_{\varphi}^{2} D_{m}
$$

As a consequence, it follows that $\left|T_{1}\right|=O_{P}\left(D_{m}\right)$.

A bound for $T_{2}$ is given by the following inequality,

$$
E\left(\mid T_{2} \| \mathcal{F}_{1}^{T}\right) \leq \frac{1}{N T} \sum_{i=1}^{N} E\left(\left\|L_{i}\right\|_{2}^{2} \mid \mathcal{F}_{1}^{T}\right)=O_{P}\left(D_{m}^{2}\right) .
$$

So (S.25) becomes $V_{N T}-V_{N T m}=O_{P}\left(D_{m}\right)=o_{P}(1)$. Hence $A_{N T}=A_{N T m}+o_{P}(1)=O_{P}(1)$, where last equality is from (S.24). Proof completed.

Proof of Lemma A.8. The proof of this lemma is based on Lyapunov C.L.T. Let $c_{i}=A_{N T m} P \Phi_{i} \phi_{m} /(N T)$. We have

$$
\sqrt{N T} A_{N T m}\left(\frac{1}{N T} \sum_{i=1}^{N} \phi_{m}^{\prime} \Phi_{i}^{\prime} P \epsilon_{i}\right)=\sum_{i=1}^{N} \sqrt{N T} c_{i}^{\prime} \epsilon_{i} .
$$

Since $c_{i} \in \mathcal{D}_{1}^{T}$ and $\epsilon_{i}$ is independent of $\mathcal{D}_{1}^{T}$, it follows that

$$
\begin{aligned}
E\left[\left(\sum_{i=1}^{N} \sqrt{N T} c_{i}^{\prime} \epsilon_{i}\right)^{2} \| \mathcal{D}_{1}^{T}\right] & =N T \sigma_{\epsilon}^{2} \sum_{i=1}^{N} c_{i}^{\prime} c_{i} \\
& =N T \sigma_{\epsilon}^{2} A_{N T m}^{2} \frac{1}{N^{2} T^{2}} \sum_{i=1}^{N} \phi_{m}^{\prime} \Phi_{i}^{\prime} P \Phi_{i} \phi_{m} \\
& =\sigma_{\epsilon}^{2} .
\end{aligned}
$$


Let $c_{i t}$ be the $t$ th element of $c_{i}$. By direct examinations, it follows that

$$
\begin{aligned}
\sum_{i=1}^{N} E\left[\left(\sqrt{N T} c_{i}^{\prime} \epsilon_{i}\right)^{4} \| \mathcal{D}_{1}^{T}\right]= & N^{2} T^{2} \sum_{i=1}^{N} \sum_{t=1}^{T} c_{i t}^{4} E\left(\epsilon_{i t}^{4}\right) \\
& +3 N^{2} T^{2} \sum_{i=1}^{N} \sum_{t=1}^{T} \sum_{t^{\prime} \neq t} c_{i t}^{2} c_{i t^{\prime}}^{2} E\left(\epsilon_{i t}^{2} \epsilon_{i t^{\prime}}^{2}\right) .
\end{aligned}
$$

Next we are going to find a bound for $c_{i t}$. By direct calculation, we have

$$
\begin{aligned}
\left|c_{i t}\right| & =\left|A_{N T m} \frac{1}{N T} p_{t} . \Phi_{i} \phi_{m}\right| \\
& \leq\left\|A_{N T m} \phi_{m}\right\|_{2}\left\|\frac{1}{N T} \sum_{s=1}^{T} p_{t s} \Phi_{i, s} \cdot\right\|_{2} \\
& \leq \lambda_{\min }^{-1 / 2}\left(H_{N T m}\right) \frac{1}{N T}\left\|\sum_{s=1}^{T} p_{t s} \Phi_{i, s} \cdot\right\|_{2},
\end{aligned}
$$

where $p_{t}$. is the th row of $P, p_{t s}$ is the $(t, s)$ th element of $P$ and $\Phi_{i, s}$. is the sth row of $\Phi_{i}$. Meanwhile, $p_{t s}=\delta_{t s}-Z_{s}^{\prime}\left(\Sigma \Sigma^{\prime}\right)^{-1} Z_{t}$, hence

$$
\begin{aligned}
& \left\|\sum_{s=1}^{T} p_{t s} \Phi_{i, s} \cdot\right\|_{2}=\left\|\Phi_{i, t}-\frac{1}{T} Z_{t}^{\prime}\left(\frac{\Sigma \Sigma^{\prime}}{T}\right)^{-1} \sum_{s=1}^{T} Z_{s} \Phi_{i, s} \cdot\right\|_{2} \\
& \leq\left\|\Phi_{i, t \cdot}\right\|_{2}+\left\|Z_{t}\right\|_{2}\left\|\left(\frac{\Sigma \Sigma^{\prime}}{T}\right)^{-1}\right\|_{\mathrm{op}} \sqrt{\frac{1}{T} \sum_{s=1}^{T}\left\|Z_{s}\right\|_{2}^{2}} \sqrt{\frac{1}{T} \sum_{s=1}^{T}\left\|\Phi_{i, s} \cdot\right\|_{2}^{2}} \\
& \leq \sqrt{m c_{\varphi}^{2}}+\left\|Z_{t}\right\|_{2}\left\|\left(\frac{\Sigma \Sigma^{\prime}}{T}\right)^{-1}\right\|_{\mathrm{op}} \sqrt{\frac{1}{T} \sum_{s=1}^{T}\left\|Z_{s}\right\|_{2}^{2}} \sqrt{m c_{\varphi}^{2}} \\
& \leq \sqrt{m c_{\varphi}^{2}}\left(1+b\left\|Z_{t}\right\|_{2}\right),
\end{aligned}
$$

where $b=\left\|\left(\frac{\Sigma \Sigma^{\prime}}{T}\right)^{-1}\right\|_{\mathrm{op}} \sqrt{\frac{1}{T} \sum_{s=1}^{T}\left\|Z_{s}\right\|_{2}^{2}}=O_{P}(1)$ by Assumption A5. So $\left|c_{i t}\right| \leq a\left(1+b\left\|Z_{t}\right\|_{2}\right)$, where $a=\lambda_{\min }^{-1 / 2}\left(H_{N T m}\right) \frac{1}{N T} \sqrt{m c_{\varphi}^{2}}$. By Lemma A.6, we have

$$
\sum_{i=1}^{N} \sum_{t=1}^{T} c_{i t}^{4} \leq \sum_{i=1}^{N} \sum_{t=1}^{T} 8 a^{4}\left(1+b^{4}\left\|Z_{t}\right\|_{2}^{4}\right)=O_{P}\left(\frac{m^{2}}{N^{3} T^{3}}\right),
$$

and

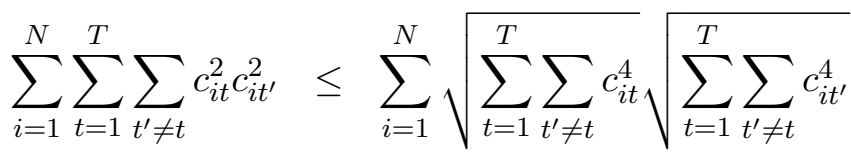

$$
\begin{aligned}
& \leq T \sum_{i=1}^{N} \sum_{t=1}^{T} c_{i t}^{4} \\
& =O_{P}\left(\frac{m^{2}}{N^{3} T^{2}}\right) \text {. }
\end{aligned}
$$


Combining (S.26), (S.27) and (S.28), we have $\sum_{i=1}^{N} E\left[\left(\sqrt{N T} c_{i}^{\prime} \epsilon_{i}\right)^{4} \| \mathcal{D}_{1}^{T}\right]=O_{P}\left(m^{2} / N\right)$. And by Lyapunov C.L.T, the result follows. Proof completed. 